\title{
CuH-Catalyzed Synthesis of 3-Hydroxyindolines and
}

\section{2-Aryl-3H-indol-3-ones from o-Alkynylnitroarenes Using Nitro as}

\section{both Nitrogen and Oxygen Source}

Hui Peng, ${ }^{a}$ Jinhui Ma, ${ }^{\mathrm{b}}$ Lingfei Duan, ${ }^{\mathrm{a}}$ Guangwen Zhang ${ }^{\mathrm{b}}$ and Biaolin Yin*a

${ }^{a}$ Key Laboratory of Functional Molecular Engineering of Guangdong Province, School of Chemistry and Chemical Engineering, South China University of Technology, Guangzhou, P.R. China, 510640

${ }^{b}$ Department of Food Science and Engineering, Jinan University, Guangzhou 510632, P.R. China

\section{Content}

General information

Experimental procedure

Reaction mechanism for the formation of $\mathbf{3}$

Characterization data of products

CIF information of $\mathbf{2 a}$

${ }^{1} \mathrm{H}$ and ${ }^{13} \mathrm{C}$ NMR spectra of all products
Page

S2

S2-S5

S6

S8-S18

S19

S20-S50 


\section{General information}

Unless specified otherwise, all experiments were performed under $\mathrm{N}_{2}$ atmosphere. All reagents and starting materials were purchased from commercial suppliers and used as received. FT-IR spectra were obtained with thin film samples or $\mathrm{KBr}$ pellets on a Bruker Vector 22 spectrometer, and data are expressed in $\mathrm{cm}^{-1} .{ }^{1} \mathrm{H},{ }^{13} \mathrm{CNMR}$ spectra were recorded on a Bruker AVANCE III $400\left(400 \mathrm{MHz}\right.$ for ${ }^{1} \mathrm{H}$; $100 \mathrm{MHz}$ for $\left.{ }^{13} \mathrm{C}\right),{ }^{1} \mathrm{H}(400 \mathrm{MHz})$ and ${ }^{13} \mathrm{C}(101 \mathrm{MHz}) \mathrm{NMR}$ spectra were recorded in $\mathrm{CDCl}_{3}$ as a solvent. Chemical shifts are reported in ppm downfield from tetramethylsilane. MS were performed on Bruker Agilent1100/Esquire HCT PLUS mass spectrometer. The HRMS measurements were recorded on a TOF analyzer using an ESI source in the positive mode. The crystals were collected at $100 \mathrm{~K}$ on a Rigaku Oxford Diffraction Supernova Dual Source, $\mathrm{Cu}$ at Zero equipped with an AtlasS2 CCD using $\mathrm{Cu} \mathrm{K} \alpha$ radiation. Column chromatography was performed on silica gel (100-200 mesh) with mixtures of petroleum ether and ethyl acetate as the eluent.

\section{Experimental procedure}

\section{Reaction Conditions Optimization ${ }^{a}$}

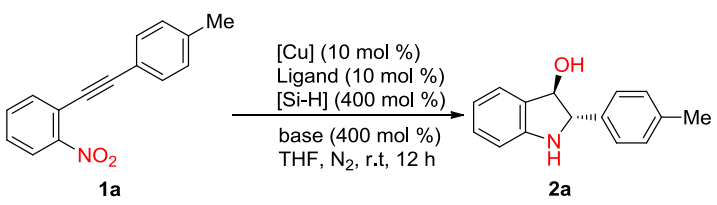

$\begin{array}{llllll}\text { entry } & {[\mathrm{Cu}]} & \text { ligand } & {[\mathrm{Si}-\mathrm{H}]} & \text { base } & \text { yield }^{b} \\ 1 & \mathrm{CuCl} & \text { dppf } & \mathrm{Me}(\mathrm{EtO})_{2} \mathrm{SiH} & \mathrm{LiO} t-\mathrm{Bu} & 27 \\ 2 & \mathrm{CuBr} & \text { dppf } & \mathrm{Me}(\mathrm{EtO})_{2} \mathrm{SiH} & \mathrm{LiO} t-\mathrm{Bu} & 30 \\ 3 & \mathrm{CuI} & \text { dppf } & \mathrm{Me}(\mathrm{EtO})_{2} \mathrm{SiH} & \mathrm{LiO} t-\mathrm{Bu} & 19 \\ 4 & \mathrm{CuOAc} & \text { dppf } & \mathrm{Me}(\mathrm{EtO})_{2} \mathrm{SiH} & \mathrm{LiO} t-\mathrm{Bu} & 8 \\ 5 & \mathrm{Cu}(\mathrm{OAc})_{2} & \text { dppf } & \mathrm{Me}(\mathrm{EtO})_{2} \mathrm{SiH} & \mathrm{LiO} t-\mathrm{Bu} & 7 \\ 6 & \mathrm{CuCl}_{2} & \text { dppf } & \mathrm{Me}(\mathrm{EtO})_{2} \mathrm{SiH} & \mathrm{LiO} t-\mathrm{Bu} & 35 \\ 7 & \mathrm{CuF}_{2} & \text { dppf } & \mathrm{Me}(\mathrm{EtO})_{2} \mathrm{SiH} & \mathrm{LiO} t-\mathrm{Bu} & 18 \\ \mathbf{8} & \mathrm{CuCl}_{2} & \text { dppf } & \mathrm{Me}(\mathrm{EtO})_{2} \mathrm{SiH} & \mathrm{LiOMe} & \mathbf{6 2} \\ 9 & \mathrm{CuCl}_{2} & \text { dppf } & \mathrm{Me}(\mathrm{EtO})_{2} \mathrm{SiH} & \mathrm{NaOMe} & 19 \\ 10 & \mathrm{CuCl}_{2} & \text { dppf } & \mathrm{Me}(\mathrm{EtO})_{2} \mathrm{SiH} & \mathrm{NaO} t-\mathrm{Bu} & \mathrm{ND} \\ 11 & \mathrm{CuCl}_{2} & \text { dppf } & \mathrm{Me}(\mathrm{EtO})_{2} \mathrm{SiH} & \mathrm{KO} t \text {-Bu } & \text { trace } \\ 12 & \mathrm{CuCl}_{2} & \text { dppe } & \mathrm{Me}(\mathrm{EtO})_{2} \mathrm{SiH} & \mathrm{LiOMe} & 17\end{array}$




\begin{tabular}{|c|c|c|c|c|c|}
\hline 13 & $\mathrm{CuCl}_{2}$ & BINAP & $\mathrm{Me}(\mathrm{EtO})_{2} \mathrm{SiH}$ & $\mathrm{LiOMe}$ & 15 \\
\hline 14 & $\mathrm{CuCl}_{2}$ & $\mathrm{PPh}_{2} \mathrm{Cy}$ & $\mathrm{Me}(\mathrm{EtO})_{2} \mathrm{SiH}$ & LiOMe & 24 \\
\hline 15 & $\mathrm{CuCl}_{2}$ & L1 & $\mathrm{Me}(\mathrm{EtO})_{2} \mathrm{SiH}$ & LiOMe & 16 \\
\hline 16 & $\mathrm{CuCl}_{2}$ & $\mathbf{L 2}$ & $\mathrm{Me}(\mathrm{EtO})_{2} \mathrm{SiH}$ & $\mathrm{LiOMe}$ & 20 \\
\hline 17 & $\mathrm{CuCl}_{2}$ & $\mathbf{L 3}$ & $\mathrm{Me}(\mathrm{EtO})_{2} \mathrm{SiH}$ & $\mathrm{LiOMe}$ & 16 \\
\hline 18 & $\mathrm{CuCl}_{2}$ & L4 & $\mathrm{Me}(\mathrm{EtO})_{2} \mathrm{SiH}$ & LiOMe & 15 \\
\hline 19 & $\mathrm{CuCl}_{2}$ & L5 & $\mathrm{Me}(\mathrm{EtO})_{2} \mathrm{SiH}$ & $\mathrm{LiOMe}$ & trace \\
\hline 20 & $\mathrm{CuCl}_{2}$ & L6 & $\mathrm{Me}(\mathrm{EtO})_{2} \mathrm{SiH}$ & $\mathrm{LiOMe}$ & trace \\
\hline 21 & $\mathrm{CuCl}_{2}$ & $\mathrm{PPh}_{3}$ & $\mathrm{Me}(\mathrm{EtO})_{2} \mathrm{SiH}$ & $\mathrm{LiOMe}$ & trace \\
\hline 22 & $\mathrm{CuCl}_{2}$ & dppf & $\mathrm{Et}_{3} \mathrm{SiH}$ & $\mathrm{LiOMe}$ & NR \\
\hline 23 & $\mathrm{CuCl}_{2}$ & dppf & {$\left[\left(\mathrm{CH}_{3}\right)_{3} \mathrm{SiO}\right]_{2} \mathrm{CH}_{3} \mathrm{SiH}$} & $\mathrm{LiOMe}$ & trace \\
\hline 24 & $\mathrm{CuCl}_{2}$ & dppf & {$\left[\left(\mathrm{CH}_{3}\right)_{2} \mathrm{SiH}\right]_{2} \mathrm{NH}$} & LiOMe & ND \\
\hline 25 & $\mathrm{CuCl}_{2}$ & dppf & $(\mathrm{EtO})_{3} \mathrm{SiH}$ & $\mathrm{LiOMe}$ & 26 \\
\hline 26 & $\mathrm{CuCl}_{2}$ & dppf & $\left(\mathrm{CH}_{3} \mathrm{O}\right)_{3} \mathrm{SiH}$ & $\mathrm{LiOMe}$ & trace \\
\hline
\end{tabular}

${ }^{a}$ Reaction conditions: 1 a $(1 \mathrm{mmol}), \mathrm{THF}(2 \mathrm{~mL}), \mathrm{NR}=$ not reaction, $\mathrm{ND}=$ not detected, $\mathrm{N}_{2}$ atmosphere, $\mathrm{rt}=$ room temperature. ${ }^{b}$ Yields were determined by ${ }^{1} \mathrm{H}$ NMR using $\mathrm{CH}_{2} \mathrm{Br}_{2}$ as the internal standard.

Detailed control experiments:

(a)

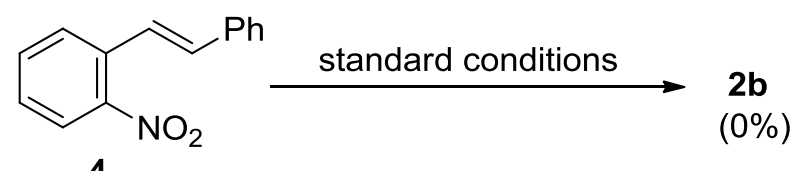

Control experiment (a): Subjecting 1-nitro-2-styrylbenzene (4) to the standard conditions did not afford $\mathbf{2 b}$, indicating that $\mathbf{4}$ was not the intermediate during the transformation of $\mathbf{1 b}$ into $\mathbf{2 b}$.

(b)

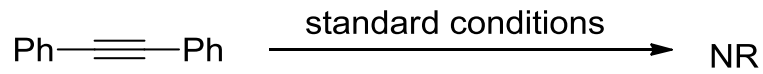

Control experiment (b): The triple bond of diphenylacetylene was not hydrogenated under the standard conditions.

(c)<smiles>O=C1C(=O)[N+]([O-])c2ccccc21</smiles>
standard conditions $5 b$

Control experiment (c): Isatogen $\mathbf{5 b}$ was transformed into $\mathbf{2 b}$ in $58 \%$ yield under the standard 
conditions, indicating that $\mathbf{5 b}$ was the possible intermediate during the transformation of $\mathbf{1 b}$ into $2 \mathbf{b}$.

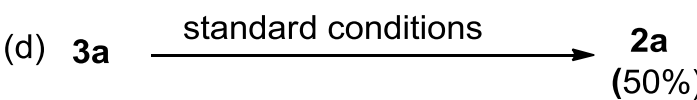

Control experiment (d): Subjecting 3a to the standard conditions afforded 2a in 50\% yield, indicating that 3a was the possible intermediate and could be reduced by silane and (or) LCuH during the transformation of $\mathbf{1 a}$ into $\mathbf{2 a}$.

(e) 3a $\underset{\operatorname{LiOMe}(400 \mathrm{~mol} \%), \mathrm{rt}, 12 \mathrm{~h}}{\stackrel{\mathrm{Me}(\mathrm{EtO})}{2} \mathrm{SiH}(400 \mathrm{~mol} \%)} \underset{(51 \%)}{2 a}$

Control experiment (e): The reaction of 3a with $\mathrm{Me}(\mathrm{EtO}){ }_{2} \mathrm{SiH}$ and $\mathrm{LiOMe}$ gave $\mathbf{2 a}$ in $51 \%$ yield. The result indicated that $3 \mathbf{a}$ could be reduced into $\mathbf{2 a}$ by silane solely in the presence of LiOMe.

(f) 1 b $\frac{\mathrm{CuCl}_{2} \text { or } \mathrm{CuCl} \text { or } \mathrm{Cu}(\mathrm{Tc})(10 \mathrm{~mol} \%)}{\mathrm{dppf}(10 \mathrm{~mol} \%), \mathrm{THF}, \mathrm{N}_{2}, \mathrm{rt}, 12 \mathrm{~h}} \longrightarrow \mathrm{NR}$

Control experiment (f): No reaction occurred when $\mathbf{1 b}$ was treated with $\mathrm{Cu}$ (I) source $(\mathrm{CuCl}$, $\mathrm{CuCl}_{2}, \mathrm{Cu}(\mathrm{Tc})$ in absence of silane. In contrast, the results in entries 1 and 6 in Table 1, demonstrated that in the presence of silane and $\mathrm{CuCl}\left(\mathrm{CuCl}_{2}\right)$, the reaction occurred and $\mathbf{2 b}$ was produced. These results indicate that $\mathrm{CuCl}, \mathrm{CuCl}_{2}, \mathrm{CuCldppf}, \mathrm{CuCl}_{2} \mathrm{dppf}, \mathrm{Cu}(\mathrm{Tc})$ cannot initiate the reaction, but $\mathrm{LCuH}$ resulting from the reaction of $\mathrm{CuCl}\left(\mathrm{CuCl}_{2}\right)$ with silane can initiate the reaction.

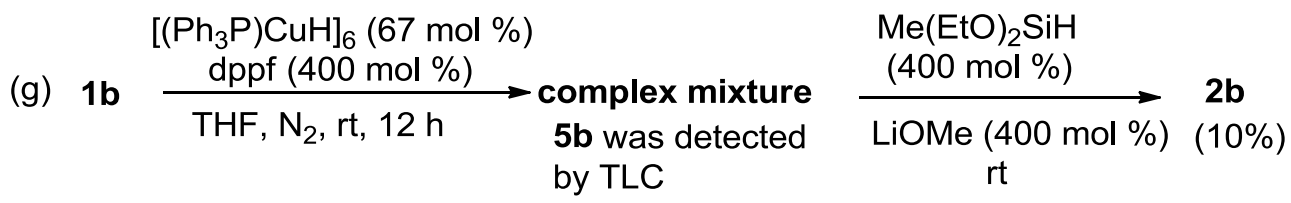

Control experiment $(\mathrm{g})$ : Treatment of $\mathbf{1 b}$ with Stryker's reagent $\left(\left[\left(\mathrm{Ph}_{3} \mathrm{P}\right) \mathrm{CuH}\right]_{6}\right)$ and dppf resulted in a complex mixtures containing a trace amount of $\mathbf{5 b}$ detected by TLC. Further treatment of the mixtures with $\mathrm{Me}(\mathrm{EtO})_{2} \mathrm{SiH}$ produced $\mathbf{2 b}$ in $10 \%$ yield. These results indicated again that $\mathrm{LCuH}$ could initiate the reaction and the complex mixture could be reduced into $\mathbf{2} \mathbf{b}$ in the presence of silane, albeit in a low yield. 
(h)

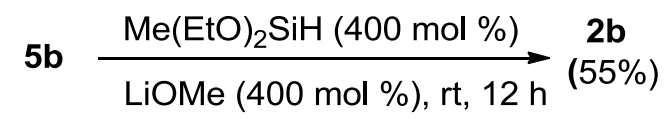

Control experiment (h): The reaction of $\mathbf{5 b}$ with $\mathrm{Me}(\mathrm{EtO})_{2} \mathrm{SiH}$ and $\mathrm{LiOMe}$ gave $\mathbf{2 b}$ in $\mathbf{5 5 \%}$ yield.

The result indicate that the nitrone and $\mathrm{C}=\mathrm{O}$ groups of $\mathbf{5 b}$ can be reduced by silane solely.

(i) 5 b $\stackrel{\mathrm{Me}(\mathrm{EtO})_{2} \mathrm{SiH}(400 \mathrm{~mol} \%)}{\longrightarrow}$ complex mixture $\mathrm{rt}, 12 \mathrm{~h}$

Control experiment $\mathbf{i}$ : The reaction of $\mathbf{5 b}$ with silane resulted in complex mixture. Compared with control experiment $\mathrm{h}$, the result indicated that the LiOMe play an important role in the process of reduction of $\mathbf{5 b}$.

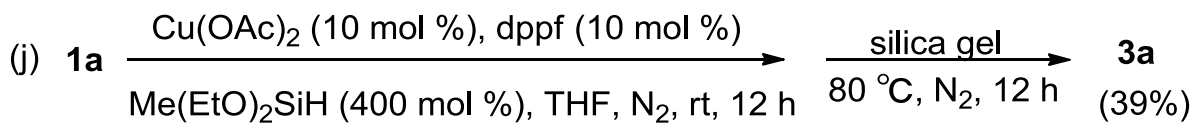

Control experiment $(\mathrm{j})$ : In the absence a base and under $\mathrm{N}_{2}$ atmosphere, upon treatment of $\mathbf{1 a}$ with $\mathrm{Cu}(\mathrm{OAc})_{2}$, dppf, $\mathrm{Me}(\mathrm{EtO})_{2} \mathrm{SiH}$, an instable product was formed, which can be further transformed into 3a in 39\% yield in the presence of silica gel, albeit in a lower yield than that under $\mathrm{O}_{2}(39 \%$ vs $46 \%)$. We inferred that the instable product was E. It suggested that $\mathbf{3 a}$ can be mainly produced by the elimination of $\mathbf{E}$ with silica gel as the promoter.

(k) 1a $\underset{\mathrm{Me}(\mathrm{EtO})_{2} \mathrm{SiH}(400 \mathrm{~mol} \%), \mathrm{THF}, \mathrm{N}_{2}, \mathrm{rt}, 12 \mathrm{~h}}{\stackrel{\mathrm{Cu}(\mathrm{OAc})_{2}(10 \mathrm{~mol} \%), \mathrm{dppf}(10 \mathrm{~mol} \%)}{\longrightarrow}} \stackrel{\text { LiOMe (400 mol \%) }}{3 a}$

Control experiment $(\mathrm{k})$ : In the absence a base, upon treatment of $\mathbf{1 a}$ with $\mathrm{Cu}(\mathrm{OAc})_{2}$, dppf, $\mathrm{Me}(\mathrm{EtO})_{2} \mathrm{SiH}$, an instable product was formed, which can be transformed into 3a in $28 \%$ yield in the presence of LiOMe. We inferred that the instable product was $\mathbf{E}$ which could also underwent reductive elimination to give $\mathbf{3 a}$, with $\mathrm{LiOMe}$ as the promoter. 


\section{Possible mechanism for the formation of 3}

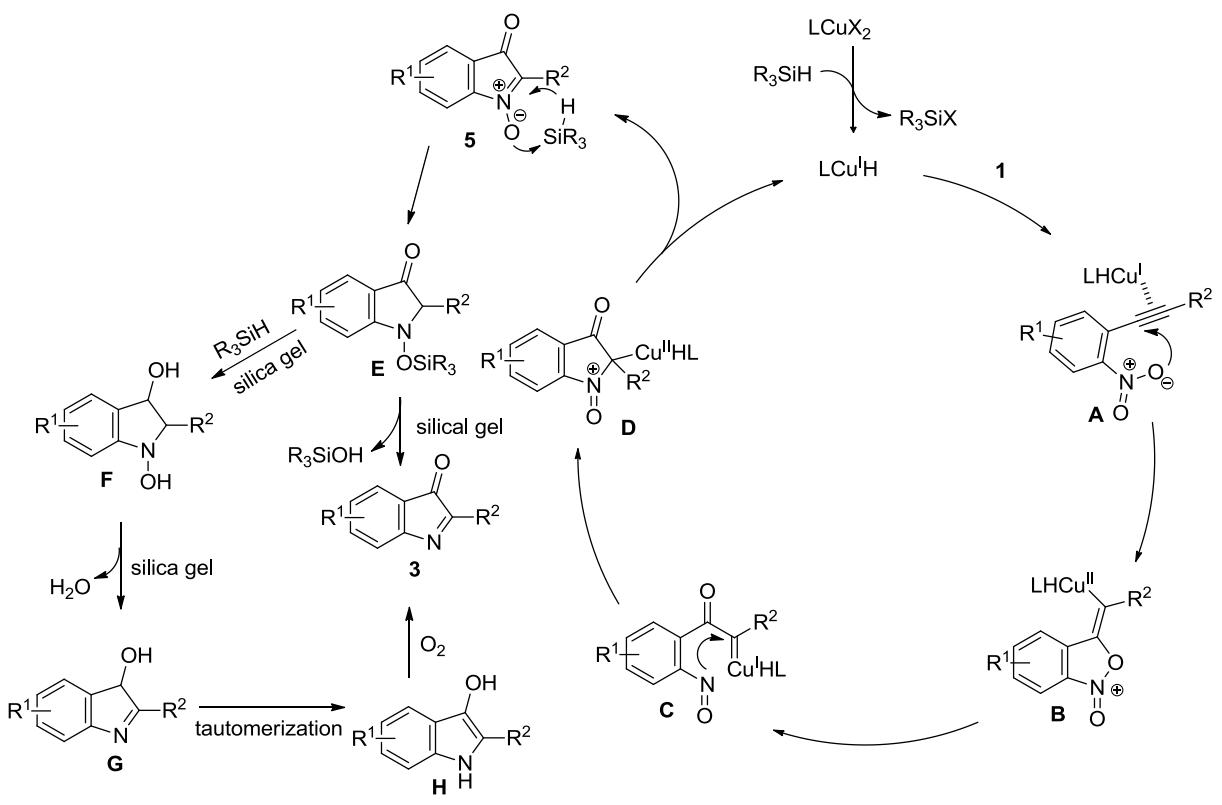

\section{General procedure for the preparation of 2}

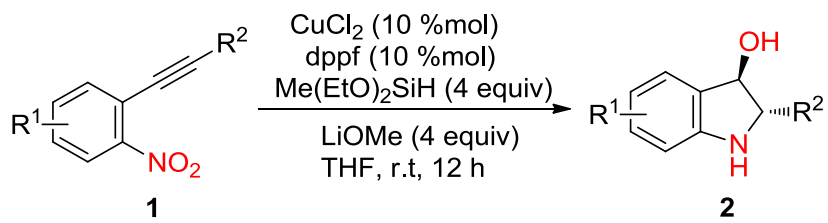

To a stirred solution of $1(0.5 \mathrm{mmol})$ in dry THF $(2 \mathrm{~mL})$ were added $\mathrm{CuCl}_{2}(6.7 \mathrm{mg}, 0.05 \mathrm{mmol}$, $10 \mathrm{~mol} \%)$, dppf (28 mg, $0.05 \mathrm{mmol}, 10 \mathrm{~mol} \%)$, LiOMe (76 mg, $2 \mathrm{mmol}, 4$ equiv) and $\mathrm{Me}(\mathrm{EtO})_{2} \mathrm{SiH}$ (268.5 mg, 2 mmol, 4 equiv) subsequently in a Schlenk flask under nitrogen atmosphere. After being stirred at room temperature for $12 \mathrm{~h}$, The resulting mixture was extracted with AcOEt $(3 \times 5 \mathrm{~mL})$. The combined organic extracts were washed with brine, dried over $\mathrm{Na}_{2} \mathrm{SO}_{4}$, filtered and concentrated. The residue was purified by flash chromatography on a silica gel (using petroleum ether/ethyl acetate $=5: 1$ as the eluent) to give product 2 .

\section{General procedure for the preparation of 3}

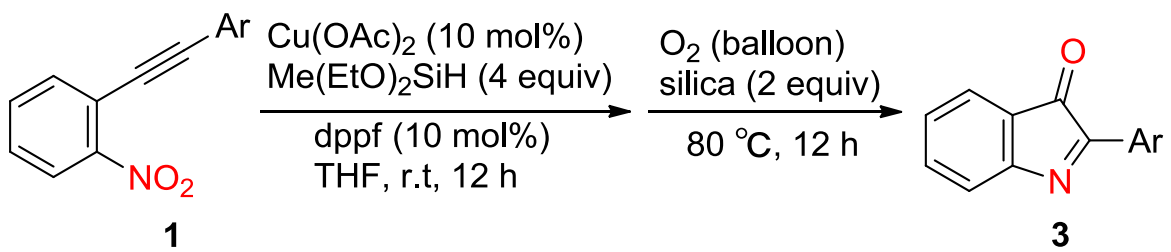

To a stirred solution of $\mathbf{1}(0.5 \mathrm{mmol})$ in dry THF $(2 \mathrm{~mL})$ were added $\mathrm{Cu}(\mathrm{OAc})_{2}(9 \mathrm{mg}, 0.05 \mathrm{mmol}$, $10 \mathrm{~mol} \%)$, dppf (28 mg, $0.05 \mathrm{mmol}, 10 \mathrm{~mol} \%)$ and $\mathrm{Me}(\mathrm{EtO})_{2} \mathrm{SiH}(268.5 \mathrm{mg}, 2 \mathrm{mmol}, 4$ equiv) 
subsequently in a Schlenk flask under nitrogen atmosphere. After being stirred at room temperature for 12 hours, silica gel $(0.06 \mathrm{~g})$ was added and the mixture reacts in an oxygen atmosphere at $80{ }^{\circ} \mathrm{C}$ for 12 hours. The resulting mixture was extracted with AcOEt $(3 \times 5 \mathrm{~mL})$. The combined organic extracts were washed with brine, dried over $\mathrm{Na}_{2} \mathrm{SO}_{4}$, filtered and concentrated. The residue was purified by flash chromatography on a silica gel (using petroleum ether/ethyl acetate $=20: 1$ as the eluent) to give product 3 .

\section{Synthesis of (E)-1-Nitro-2-styrylbenzene 4.}

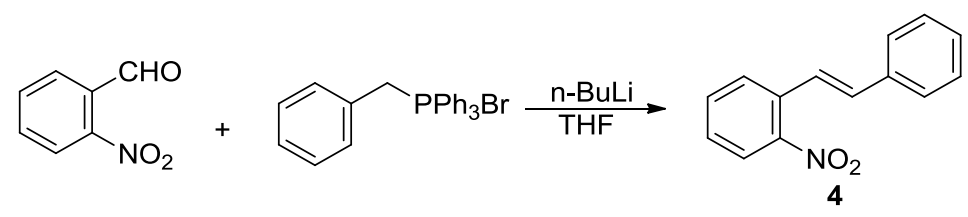

To a solution of the (Phenylmethyl)triphenylphosphonium bromide (3.5 mmol) in anhydrous THF $(10 \mathrm{~mL})$ at $-78{ }^{\circ} \mathrm{C}$ was added slowly $\mathrm{n}-\mathrm{BuLi}(1.9 \mathrm{~mL}$ of a $1.6 \mathrm{M}$ solution in hexane, $3.0 \mathrm{mmol})$. The resulting mixture was stirred for $2 \mathrm{~h}$ and then 2-nitrobenzaldehyde $(3.5 \mathrm{mmol})$ in THF (10 $\mathrm{mL})$ was added dropwise. The resulting solution was stirred for $1 \mathrm{~h}$ at $-78{ }^{\circ} \mathrm{C}$ and then at room temperature for $12 \mathrm{~h}$. The mixture was poured into water, and the resulting mixture was extracted with $3 \times 10 \mathrm{~mL}$ of ethyl acetate, and the combined organic layers were washed with brine, dried over anhydrous $\mathrm{Na}_{2} \mathrm{SO}_{4}$, and evaporated under reduced pressure. The residue was purified by flash chromatography on a silica gel (using petroleum ether/ethyl acetate $=10: 1$ as the eluent) to give product 4 .

\section{Synthesis of 3-oxo-2-phenyl-3H-indole 1-oxide $5 \mathrm{~b}$.}

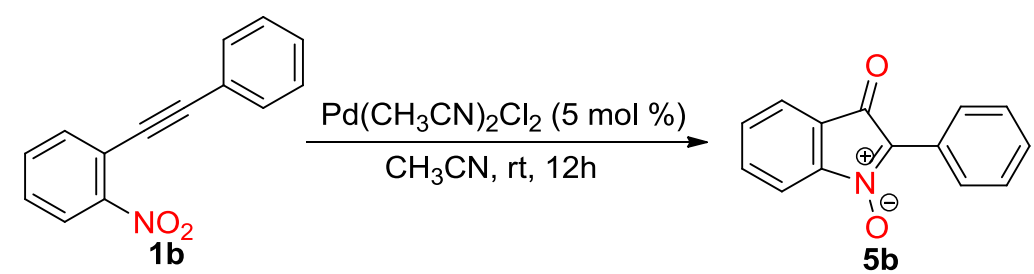

To a stirred solution of $\mathbf{1 b}(0.5 \mathrm{mmol})$ in dry $\mathrm{CH}_{3} \mathrm{CN}(2 \mathrm{~mL})$ were added $\mathrm{Pd}\left(\mathrm{CH}_{3} \mathrm{CN}\right)_{2} \mathrm{Cl}_{2}(6.5 \mathrm{mg}$, $0.025 \mathrm{mmol}, 5 \mathrm{~mol} \%$ ) in a Schlenk flask under nitrogen atmosphere. After being stirred at room temperature for $12 \mathrm{~h}$, The resulting mixture was extracted with AcOEt $(3 \times 5 \mathrm{~mL})$. The combined organic extracts were washed with brine, dried over $\mathrm{Na}_{2} \mathrm{SO}_{4}$, filtered and concentrated. The residue was purified by flash chromatography on a silica gel (using petroleum ether/ethyl acetate $=10: 1$ as the eluent) to give product $\mathbf{5 b}$.

\section{Procedure for control experiments}


(f)<smiles>O=[N+]([O-])c1ccccc1C#Cc1ccccc1</smiles>

1b

$\left[\left(\mathrm{Ph}_{3} \mathrm{P}\right) \mathrm{CuH}\right]_{6}(67 \mathrm{~mol} \%)$

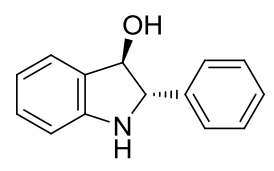

2b $(10 \%)$

A $25 \mathrm{~mL}$ Schlenk flask was charged with $\left[\left(\mathrm{Ph}_{3} \mathrm{P}\right) \mathrm{CuH}\right]_{6}(0.65 \mathrm{~g}, 0.33 \mathrm{mmol}, 67 \mathrm{~mol} \%)$ and dppf (1.1 g, $2 \mathrm{mmol}, 400 \mathrm{~mol} \%)$. THF ( $2 \mathrm{~mL})$ was then added and the mixture was stirred for 2 hours before the addition of $\mathbf{1 b}(0.5 \mathrm{mmol})$. After the reaction being stirred at room temperature for 12 hours, $\mathrm{Me}(\mathrm{EtO})_{2} \mathrm{SiH}$ (268.5 mg, $2 \mathrm{mmol}, 4$ equiv) and LiOMe (76 mg, $2 \mathrm{mmol}, 4.0$ equiv) were added and the mixture reacts in a nitrogen atmosphere at $80{ }^{\circ} \mathrm{C}$ for 12 hours. Subsequently, the precipitate was removed by filtration. The filtrate was washed with brine, dried over anhydrous $\mathrm{Na}_{2} \mathrm{SO}_{4}$, and evaporated. The desired product $\mathbf{2 b}$ was obtained in $10 \%$ NMR yield.

(g)<smiles>Nc1ccc(C#Cc2ccccc2[N+](=O)[O-])cc1</smiles>

1a
$\mathrm{Cu}(\mathrm{OAc})_{2}(10 \mathrm{~mol} \%)$ $\mathrm{Me}(\mathrm{EtO})_{2} \mathrm{SiH}(400 \mathrm{~mol} \%)$ dppf $(10 \mathrm{~mol} \%)$ THF, rt, $12 \mathrm{~h}$
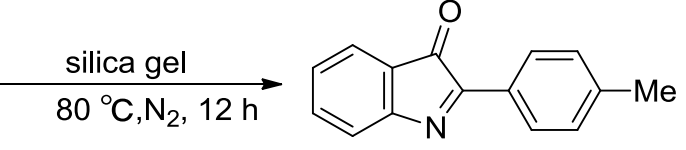

$3 a, 39 \%$

To a stirred solution of $\mathbf{1 a}(0.5 \mathrm{mmol})$ in dry THF $(2 \mathrm{~mL})$ were added $\mathrm{Cu}(\mathrm{OAc})_{2}(9 \mathrm{mg}, 0.05 \mathrm{mmol}$, $10 \mathrm{~mol} \%)$, dppf (28 mg, $0.05 \mathrm{mmol}, 10 \mathrm{~mol} \%)$ and $\mathrm{Me}(\mathrm{EtO})_{2} \mathrm{SiH}(268.5 \mathrm{mg}, 2 \mathrm{mmol}, 4$ equiv) subsequently in a Schlenk flask under nitrogen atmosphere. After the reaction being stirred at room temperature for 12 hours, silica gel $(0.06 \mathrm{~g})$ was added and the mixture reacts in a nitrogen atmosphere at $80{ }^{\circ} \mathrm{C}$ for 12 hours. The resulting mixture was extracted with AcOEt $(3 \times 5 \mathrm{~mL})$. The combined organic extracts were washed with brine, dried over $\mathrm{Na}_{2} \mathrm{SO}_{4}$, filtered and concentrated. The residue was purified by flash chromatography on a silica gel (using petroleum ether/ethyl acetate $=20: 1$ as the eluent) to give product 3a in $39 \%$ yield $(43.1 \mathrm{mg})$.

\section{Characterization Data for 2}<smiles>Cc1ccc([C@@H]2Nc3ccccc3[C@H]2O)cc1</smiles>

2-(p-tolyl)indolin-3-ol (2a); Yellow solid was obtained in 52\% (58.5 mg) isolated yield; mp 62-63 ${ }^{\circ} \mathrm{C}$; IR (KBr) v: 3368, 1391, 1315, 876, 790, 747; ${ }^{1} \mathrm{H}$ NMR $\left(400 \mathrm{MHz}, \mathrm{CDCl}_{3}\right) \delta 7.28-7.22(\mathrm{~m}$, 3H), $7.18(\mathrm{t}, J=7.6 \mathrm{~Hz}, 1 \mathrm{H}), 7.12(\mathrm{~d}, J 7.9 \mathrm{~Hz}, 2 \mathrm{H}), 6.80(\mathrm{t}, J=7.4 \mathrm{~Hz}, 1 \mathrm{H}), 6.71(\mathrm{~d}, J=7.9 \mathrm{~Hz}, 1 \mathrm{H})$, $5.01(\mathrm{~d}, J=4.9 \mathrm{~Hz}, 1 \mathrm{H}), 4.60(\mathrm{~d}, J=4.9 \mathrm{~Hz}, 1 \mathrm{H}), 4.09(\mathrm{br}, 1 \mathrm{H}), 2.32(\mathrm{~s}, 3 \mathrm{H}) ;{ }^{13} \mathrm{C} \mathrm{NMR}(101 \mathrm{MHz}$, 
$\left.\mathrm{CDCl}_{3}\right) \delta 150.48,139.21,137.42,129.92,129.41,129.00,126.22,125.27,119.23,109.76,81.40,72.17$ 21.09; HRMS (ESI) $m / z$ calcd for $\mathrm{C}_{15} \mathrm{H}_{16} \mathrm{NO}[\mathrm{M}+\mathrm{H}]^{+} 226.1226$, Found 226.1228.<smiles>O[C@@H]1c2ccccc2N[C@H]1c1ccccc1</smiles>

2-phenylindolin-3-ol (2b); Yellow syrup was obtained in 62\% (65 mg) isolated yield; IR (KBr) v: 3442, 1315, 1176, 745, 603; ${ }^{1} \mathrm{H}$ NMR (400 MHz, $\left.\mathrm{CDCl}_{3}\right) \delta 7.39-7.35$ (m, 2H), 7.35 - 7.26 (m, 4H), $7.22-7.17(\mathrm{~m}, 1 \mathrm{H}), 6.81(\mathrm{t}, J=7.4 \mathrm{~Hz}, 1 \mathrm{H}), 6.74(\mathrm{~d}, J=7.9 \mathrm{~Hz}, 1 \mathrm{H}), 5.05(\mathrm{~d}, J=5.0 \mathrm{~Hz}, 1 \mathrm{H}), 4.67(\mathrm{~d}$, $J=5.0 \mathrm{~Hz}, 1 \mathrm{H}), 4.20(\mathrm{br}, 1 \mathrm{H}) ;{ }^{13} \mathrm{C} \mathrm{NMR}\left(101 \mathrm{MHz}, \mathrm{CDCl}_{3}\right) \delta 150.44,142.22,129.98,128.87,128.75$, 127.73, 126.27, $125.25,119.28,109.75,81.41,72.42$; HRMS (ESI) $m / z$ calcd for $\mathrm{C}_{14} \mathrm{H}_{14} \mathrm{NO}[\mathrm{M}+\mathrm{H}]^{+}$ 212.1070, Found 212.1074.<smiles>COc1ccc([C@@H]2Nc3ccccc3[C@H]2O)cc1</smiles>

2-(4-methoxyphenyl)indolin-3-ol (2c); Yellow solid was obtained in 61\% (73 mg) isolated yield; mp 91-93 ${ }^{\circ} \mathrm{C}$; IR (KBr) v: 3446, 1299, 1246, 826, 749; ${ }^{1} \mathrm{H}$ NMR $\left(400 \mathrm{MHz}, \mathrm{CDCl}_{3}\right) \delta 7.25-7.21(\mathrm{~m}$, 3H), $7.15(\mathrm{t}, J=7.6 \mathrm{~Hz}, 1 \mathrm{H}), 6.84-6.75(\mathrm{~m}, 3 \mathrm{H}), 6.66(\mathrm{~d}, J=7.9 \mathrm{~Hz}, 1 \mathrm{H}), 4.95(\mathrm{~d}, J=4.0 \mathrm{~Hz}, 1 \mathrm{H})$, 4.51 (br, 1H), 3.75 (s, 3H), $3.28(\mathrm{br}, 1 \mathrm{H}) ;{ }^{13} \mathrm{C}$ NMR (101 MHz, $\left.\mathrm{CDCl}_{3}\right) \delta 159.18,150.41,134.34$, $129.85,129.13,127.48,125.28,119.26,114.11,109.81,81.30,71.85,55.34 ;$ HRMS $(\mathrm{ESI}) \mathrm{m} / \mathrm{z}$ calcd for $\mathrm{C}_{15} \mathrm{H}_{16} \mathrm{NO}_{2}[\mathrm{M}+\mathrm{H}]^{+} 242.1179$, Found 242.1176.<smiles>CC(C)(C)c1ccc([C@@H]2Nc3ccccc3[C@H]2O)cc1</smiles>

2-(4-(tert-butyl)phenyl)indolin-3-ol (2d); Yellow solid was obtained in 56\% (74 mg) isolated yield; $\operatorname{mp} 77-79^{\circ} \mathrm{C}$; IR (KBr) v: $3371,1363,1311,880,832,746 ;{ }^{1} \mathrm{H}$ NMR $\left(400 \mathrm{MHz}, \mathrm{CDCl}_{3}\right) \delta 7.34(\mathrm{~d}, J=$ $8.4 \mathrm{~Hz}, 2 \mathrm{H}), 7.27(\mathrm{~d}, J=8.2 \mathrm{~Hz}, 3 \mathrm{H}), 7.17(\mathrm{t}, J=7.6 \mathrm{~Hz}, 1 \mathrm{H}), 6.79(\mathrm{t}, J=7.4 \mathrm{~Hz}, 1 \mathrm{H}), 6.70(\mathrm{~d}, J=7.9$ $\mathrm{Hz}, 1 \mathrm{H}), 5.03(\mathrm{~d}, J=4.8 \mathrm{~Hz}, 1 \mathrm{H}), 4.60(\mathrm{~d}, J=4.8 \mathrm{~Hz}, 1 \mathrm{H}), 4.11(\mathrm{br}, 1 \mathrm{H}), 2.40(\mathrm{br}, 1 \mathrm{H}) 1.30(\mathrm{~s}, 9 \mathrm{H}) ;{ }^{13} \mathrm{C}$ NMR $\left(101 \mathrm{MHz}, \mathrm{CDCl}_{3}\right) \delta 150.73,150.51,139.15,129.91,129.06,126.03,125.64,125.29,119.23$, 109.78, 81.22, 72.07, 34.54, 31.37; HRMS (ESI) $\mathrm{m} / z$ calcd for $\mathrm{C}_{18} \mathrm{H}_{22} \mathrm{NO}[\mathrm{M}+\mathrm{H}]^{+} 268.1700$, Found 268.1696. 
<smiles>CCc1ccc([C@@H]2Nc3ccccc3[C@H]2O)cc1</smiles>

2-(4-ethylphenyl)indolin-3-ol (2e); Yellow solid was obtained in 56\% (67 mg) isolated yield; $\mathrm{mp}$ $66-69^{\circ} \mathrm{C}$; IR (KBr) v: $3443,1458,787,743 ;{ }^{1} \mathrm{H}$ NMR $\left(400 \mathrm{MHz}, \mathrm{CD}_{3} \mathrm{OD}\right) \delta 7.18(\mathrm{~d}, J=7.8 \mathrm{~Hz}, 2 \mathrm{H})$, $7.09(\mathrm{~d}, J=7.3 \mathrm{~Hz}, 1 \mathrm{H}), 7.05-6.96(\mathrm{~m}, 3 \mathrm{H}), 6.63-6.57(\mathrm{~m}, 2 \mathrm{H}), 4.83(\mathrm{~d}, J=5.7 \mathrm{~Hz}, 1 \mathrm{H}), 4.44(\mathrm{~d}, J=$ $5.6 \mathrm{~Hz}, 1 \mathrm{H}), 2.49(\mathrm{q}, J=7.6 \mathrm{~Hz}, 2 \mathrm{H}), 1.08(\mathrm{t}, J=7.6 \mathrm{~Hz}, 3 \mathrm{H}) ;{ }^{13} \mathrm{C} \mathrm{NMR}\left(101 \mathrm{MHz}, \mathrm{CD}_{3} \mathrm{OD}\right) \delta 151.08$, $143.24,140.19,129.36,128.98,127.57,126.14,124.58,118.17,109.34,80.90,71.73,28.16,14.91$; HRMS (ESI) $m / z$ calcd for $\mathrm{C}_{16} \mathrm{H}_{18} \mathrm{NO}[\mathrm{M}+\mathrm{H}]^{+} 240.1383$, Found 240.1383 .<smiles>COc1cccc([C@@H]2Nc3ccccc3[C@H]2O)c1</smiles>

2-(3-methoxyphenyl)indolin-3-ol (2f); Yellow solid was obtained in 52\% (62 mg) isolated yield; mp 63-65 ${ }^{\circ}$; IR (KBr) v: 3448, 1316, 1253, 745, 699; ${ }^{1} \mathrm{H}$ NMR (400 MHz, $\left.\mathrm{CDCl}_{3}\right) \delta 7.27-7.14(\mathrm{~m}$, 3H), $6.93(\mathrm{~d}, J=7.4 \mathrm{~Hz}, 2 \mathrm{H}), 6.79(\mathrm{t}, J=7.3 \mathrm{~Hz}, 2 \mathrm{H}), 6.70(\mathrm{~d}, J=7.9 \mathrm{~Hz}, 1 \mathrm{H}), 5.00(\mathrm{~d}, J=5.0 \mathrm{~Hz}$, $1 \mathrm{H}), 4.58(\mathrm{~d}, J=5.0 \mathrm{~Hz}, 1 \mathrm{H}), 4.15(\mathrm{br}, 1 \mathrm{H}), 3.75(\mathrm{~s}, 3 \mathrm{H}), 2.56(\mathrm{br}, 1 \mathrm{H}) ;{ }^{13} \mathrm{C} \mathrm{NMR}\left(101 \mathrm{MHz}, \mathrm{CDCl}_{3}\right) \delta$ $159.93,150.38,143.98,129.92,129.80,128.95,125.25,119.30,118.56,113.17,111.79,109.82,81.31$, 72.34, 55.24; HRMS (ESI) $m / z$ calcd for $\mathrm{C}_{15} \mathrm{H}_{16} \mathrm{NO}_{2}[\mathrm{M}+\mathrm{H}]^{+} 242.1176$, Found 242.1174 .<smiles>Cc1cccc([C@@H]2Nc3ccccc3C2O)c1</smiles>

2-(m-tolyl)indolin-3-ol (2g); Yellow solid was obtained in 54\% (60 mg) isolated yield; mp 79-81 ${ }^{\circ} \mathrm{C}$; IR (KBr) v: 3367, 1390, 1312, 875, 793, 747; ${ }^{1} \mathrm{H}$ NMR (400 MHz, $\left.\mathrm{CDCl}_{3}\right) \delta 7.25(\mathrm{~d}, J=8.1 \mathrm{~Hz}, 1 \mathrm{H})$, $7.21-7.07(\mathrm{~m}, 5 \mathrm{H}), 6.80-6.71(\mathrm{~m}, 2 \mathrm{H}), 4.99(\mathrm{~d}, J=3.0 \mathrm{~Hz}, 1 \mathrm{H}), 4.93(\mathrm{~d}, J=3.3 \mathrm{~Hz}, 1 \mathrm{H}), 4.13(\mathrm{br}$, 1H), 2.47 (s, 3H), $2.38(\mathrm{br}, 1 \mathrm{H}) ;{ }^{13} \mathrm{C} \mathrm{NMR}\left(101 \mathrm{MHz}, \mathrm{CDCl}_{3}\right) \delta 150.80,139.97,135.84,130.76,130.21$, 128.67, 127.28, 126.26, 125.64, 125.18, 119.14, 109.65, 80.88, 68.26, 19.68; HRMS (ESI) $\mathrm{m} / z$ calcd for $\mathrm{C}_{15} \mathrm{H}_{16} \mathrm{NO}[\mathrm{M}+\mathrm{Na}]^{+} 226.1226$, Found 226.1228 .<smiles>COc1ccccc1[C@@H]1Nc2ccccc2C1O</smiles> 
2-(o-tolyl)indolin-3-ol (2h); Yellow solid was obtained in 52\% (58 mg) isolated yield; $\mathrm{mp} 63^{\circ} \mathrm{C}$; IR (KBr) v: 3450, 1387, 1314, 744, 705; ${ }^{1} \mathrm{H}$ NMR (400 MHz, $\left.\mathrm{CDCl}_{3}\right) \delta 7.23(\mathrm{~d}, J=7.4 \mathrm{~Hz}, 1 \mathrm{H}), 7.21-$ $7.13(\mathrm{~m}, 3 \mathrm{H}), 7.11(\mathrm{~d}, J=7.6 \mathrm{~Hz}, 1 \mathrm{H}), 7.07(\mathrm{~d}, J=7.3 \mathrm{~Hz}, 1 \mathrm{H}), 6.78(\mathrm{t}, J=7.4 \mathrm{~Hz}, 1 \mathrm{H}), 6.67(\mathrm{~d}, J=$ $7.9 \mathrm{~Hz}, 1 \mathrm{H}), 4.98(\mathrm{~d}, J=4.9 \mathrm{~Hz}, 1 \mathrm{H}), 4.53(\mathrm{~d}, J=4.7 \mathrm{~Hz}, 1 \mathrm{H}), 4.10(\mathrm{br}, 1 \mathrm{H}), 2.69$ (br, 1H), $2.31(\mathrm{~s}$, $3 \mathrm{H}) ;{ }^{13} \mathrm{C}$ NMR $\left(101 \mathrm{MHz}, \mathrm{CDCl}_{3}\right) \delta 150.44,142.27,138.43,129.88,128.66,128.48,127.04,125.30$, 123.37, 119.29, 109.85, 81.34, 72.34, 21.47; HRMS (ESI) $m / z$ calcd for $\mathrm{C}_{15} \mathrm{H}_{16} \mathrm{NO}[\mathrm{M}+\mathrm{Na}]^{+} 226.1228$, Found 226.1226.<smiles>O[C@@H]1c2ccccc2N[C@H]1c1ccc(Br)cc1</smiles>

2-(4-bromophenyl)indolin-3-ol (2i); Yellow solid was obtained in 40\% (57 mg) isolated yield; mp 63-65두 $\quad$ IR (KBr) v: $3440,1395,1310,801,748 ;{ }^{1} \mathrm{H}$ NMR $\left(400 \mathrm{MHz}, \mathrm{CDCl}_{3}\right) \delta 7.43(\mathrm{~d}, J=8.2 \mathrm{~Hz}$, 2H), $7.24(\mathrm{t}, J=8.0 \mathrm{~Hz}, 3 \mathrm{H}), 7.18(\mathrm{t}, J=7.7 \mathrm{~Hz}, 1 \mathrm{H}), 6.81(\mathrm{t}, J=7.4 \mathrm{~Hz}, 1 \mathrm{H}), 6.71(\mathrm{~d}, J=7.9 \mathrm{~Hz}, 1 \mathrm{H})$, $4.95(\mathrm{~d}, J=5.2 \mathrm{~Hz}, 1 \mathrm{H}), 4.56(\mathrm{~d}, J=5.1 \mathrm{~Hz}, 1 \mathrm{H}), 3.68(\mathrm{br}, 1 \mathrm{H}), 2.80(\mathrm{br}, 1 \mathrm{H}) ;{ }^{13} \mathrm{C} \mathrm{NMR}(101 \mathrm{MHz}$, $\left.\mathrm{CDCl}_{3}\right) \delta 150.13,141.32,131.79,130.01,128.74,128.06,125.23,121.46,119.52,109.93,81.27,71.73 ;$ HRMS (ESI) $m / z$ calcd for $\mathrm{C}_{14} \mathrm{H}_{13} \mathrm{BrNO}[\mathrm{M}+\mathrm{H}]^{+} 290.0173$, Found 290.0175 .<smiles>O[C@@H]1c2ccccc2N[C@H]1c1ccc(F)cc1</smiles>

2-(4-fluorophenyl)indolin-3-ol (2j); Yellow solid was obtained in 63\% (72 mg) isolated yield; mp $163-165^{\circ} \mathrm{C}$; IR (KBr) v: 3418, 1344, 1229, 833, 793; ${ }^{1} \mathrm{H}$ NMR (400 MHz, $\left.\mathrm{CDCl}_{3}\right) \delta 7.33-7.27$ (m, 2H), $7.25(\mathrm{~d}, J=7.6 \mathrm{~Hz}, 1 \mathrm{H}), 7.17(\mathrm{t}, J=7.6 \mathrm{~Hz}, 1 \mathrm{H}), 6.98(\mathrm{t}, J=8.6 \mathrm{~Hz}, 2 \mathrm{H}), 6.80(\mathrm{t}, J=7.4 \mathrm{~Hz}, 1 \mathrm{H}), 6.70$ $(\mathrm{d}, J=7.9 \mathrm{~Hz}, 1 \mathrm{H}), 4.95(\mathrm{~d}, J=5.2 \mathrm{~Hz}, 1 \mathrm{H}), 4.57(\mathrm{~d}, J=5.2 \mathrm{~Hz}, 1 \mathrm{H}), 4.08(\mathrm{br}, 1 \mathrm{H}), 2.71(\mathrm{br}, 1 \mathrm{H}) ;{ }^{13} \mathrm{C}$ NMR $\left(101 \mathrm{MHz}, \mathrm{CDCl}_{3}\right) \delta 163.58,161.13,150.21,138.00,137.97,129.98,128.83,127.95,127.87$, 125.24, 119.47, 115.63, 115.42, 109.90, 81.37, 71.69; HRMS (ESI) $m / z$ calcd for $\mathrm{C}_{14} \mathrm{H}_{13} \mathrm{FNO}[\mathrm{M}+\mathrm{H}]^{+}$ 230.0976, Found 230.0972.<smiles>O[C@@H]1c2ccccc2N[C@H]1c1ccc(Cl)cc1</smiles>

2-(4-chlorophenyl)indolin-3-ol (2k); Yellow solid was obtained in 54\% (66 mg) isolated yield; mp $124-126^{\circ} \mathrm{C} ; \quad \mathrm{IR}(\mathrm{KBr})$ v: $3438,1422,1305,830,793,742 ;{ }^{1} \mathrm{H}$ NMR $\left(400 \mathrm{MHz}, \mathrm{CDCl}_{3}\right) \delta 7.25(\mathrm{~s}, 4 \mathrm{H})$, 
$7.22(\mathrm{~d}, J=7.4 \mathrm{~Hz}, 1 \mathrm{H}), 7.16(\mathrm{t}, J=7.6 \mathrm{~Hz}, 1 \mathrm{H}), 6.79(\mathrm{t}, J=7.4 \mathrm{~Hz}, 1 \mathrm{H}), 6.68(\mathrm{~d}, J=7.9 \mathrm{~Hz}, 1 \mathrm{H})$, $4.91(\mathrm{~d}, J=5.1 \mathrm{~Hz}, 1 \mathrm{H}), 4.52(\mathrm{~d}, J=5.1 \mathrm{~Hz}, 1 \mathrm{H}) ;{ }^{13} \mathrm{C} \mathrm{NMR}\left(101 \mathrm{MHz}, \mathrm{CDCl}_{3}\right) \delta 150.15,140.77$, 133.37, 130.01, 128.85, 128.76, 127.71, 125.27, 119.54, 109.97, 81.28, 71.64; HRMS (ESI) $\mathrm{m} / \mathrm{z}$ calcd for $\mathrm{C}_{14} \mathrm{H}_{12} \mathrm{ClNNaO}[\mathrm{M}+\mathrm{Na}]^{+}$268.0500, Found 268.0508.<smiles>O[C@H]1c2ccccc2N[C@H]1c1ccc(C(F)(F)F)cc1</smiles>

2-(4-(trifluoromethyl)phenyl)indolin-3-ol (2I); Yellow solid was obtained in 58\% (81 mg) isolated yield; mp 212-216 ${ }^{\circ}$; IR (KBr) v: $3421,1418,1320,838,795,747 ;{ }^{1} \mathrm{H}$ NMR (400 MHz, $\left.\mathrm{CD}_{3} \mathrm{OD}\right) \delta$ $7.47(\mathrm{~s}, 4 \mathrm{H}), 7.09(\mathrm{~d}, J=7.3 \mathrm{~Hz}, 1 \mathrm{H}), 6.99(\mathrm{t}, J=7.6 \mathrm{~Hz}, 1 \mathrm{H}), 6.61(\mathrm{t}, J=8.4 \mathrm{~Hz}, 2 \mathrm{H}), 4.82(\mathrm{~d}, J=6.0$ $\mathrm{Hz}, 1 \mathrm{H}), 4.55(\mathrm{~d}, J=6.0 \mathrm{~Hz}, 1 \mathrm{H}) ;{ }^{13} \mathrm{C}$ NMR $\left(101 \mathrm{MHz}, \mathrm{CD}_{3} \mathrm{OD}\right) \delta 150.79,147.75,129.30,129.14$, 128.98, 126.74, 125.76, 125.02, 124.99, 124.57, 123.07, 118.46, 109.49, 80.91, 71.50; HRMS (ESI) $m / z$ calcd for $\mathrm{C}_{15} \mathrm{H}_{13} \mathrm{~F}_{3} \mathrm{NO}[\mathrm{M}+\mathrm{Na}]^{+} 280.0944$, Found 280.0947 .<smiles>OC1c2ccccc2NC1c1cccc2ccccc12</smiles>

2-(naphthalen-1-yl)indolin-3-ol (2m); Yellow solid was obtained in 41\% (53 mg) isolated yield; $\mathrm{mp}$ $133-135^{\circ} \mathrm{C}$; IR (KBr) v: 3287, 1383, 1310, 872, 746; ${ }^{1} \mathrm{H}$ NMR $\left(400 \mathrm{MHz}, \mathrm{CD}_{3} \mathrm{OD}\right) \delta 8.38(\mathrm{~d}, J=8.1 \mathrm{~Hz}$ 1H), $7.89(\mathrm{~d}, J=7.5 \mathrm{~Hz}, 1 \mathrm{H}), 7.75(\mathrm{~d}, J=8.1 \mathrm{~Hz}, 1 \mathrm{H}), 7.55-7.49(\mathrm{~m}, 3 \mathrm{H}), 7.37(\mathrm{t}, J=7.7 \mathrm{~Hz}, 1 \mathrm{H})$, $7.23-7.15(\mathrm{~m}, 2 \mathrm{H}), 6.80(\mathrm{~d}, J=7.9 \mathrm{~Hz}, 1 \mathrm{H}), 6.73(\mathrm{t}, J=7.4 \mathrm{~Hz}, 1 \mathrm{H}), 5.41(\mathrm{~d}, J=3.7 \mathrm{~Hz}, 1 \mathrm{H}), 5.08(\mathrm{~d}$, $J=3.8 \mathrm{~Hz}, 1 \mathrm{H}) ;{ }^{13} \mathrm{C}$ NMR $\left(101 \mathrm{MHz}, \mathrm{CD}_{3} \mathrm{OD}\right) \delta 151.48,138.11,134.30,131.26,129.36,128.79$, $128.51,127.39,125.50,125.22,125.18,124.98,123.55,122.87,117.90,109.17,80.19,68.41 ;$ HRMS (ESI) $m / z$ calcd for $\mathrm{C}_{18} \mathrm{H}_{16} \mathrm{NO}[\mathrm{M}+\mathrm{H}]^{+} 262.1226$, Found 262.1229 .<smiles>O[C@@H]1c2ccccc2N[C@H]1c1ccc2ccccc2c1</smiles>

2-(naphthalen-2-yl)indolin-3-ol (2n); Yellow solid was obtained in 51\% (66.5 mg) isolated yield; mp 99-101 ${ }^{\circ} \mathrm{C}$; IR (KBr) v: 3383, 1378, 1252, 861, 817, 746; ${ }^{1} \mathrm{H}$ NMR (400 MHz, $\left.\mathrm{CD}_{3} \mathrm{OD}\right) \delta 7.86(\mathrm{~s}, 1 \mathrm{H})$, $7.80(\mathrm{t}, J=7.1 \mathrm{~Hz}, 3 \mathrm{H}), 7.55(\mathrm{~d}, J=8.6 \mathrm{~Hz}, 1 \mathrm{H}), 7.46-7.41(\mathrm{~m}, 2 \mathrm{H}), 7.26(\mathrm{~d}, J=7.6 \mathrm{~Hz}, 1 \mathrm{H}), 7.16(\mathrm{t}$, $J=7.7 \mathrm{~Hz}, 1 \mathrm{H}), 6.79-6.74(\mathrm{~m}, 2 \mathrm{H}), 5.08(\mathrm{~d}, J=5.7 \mathrm{~Hz}, 1 \mathrm{H}), 4.78(\mathrm{~d}, J=5.7 \mathrm{~Hz}, 1 \mathrm{H}) ;{ }^{13} \mathrm{C} \mathrm{NMR}(101$ 
$\left.\mathrm{MHz}, \mathrm{CD}_{3} \mathrm{OD}\right) \delta 151.09,140.42,133.53,133.09,129.27,129.08,127.93,127.47,127.27,125.71$, 125.29, 124.74, 124.64, 124.33, 118.24, 109.37, 80.81, 72.07; HRMS (ESI) $m / z$ calcd for $\mathrm{C}_{18} \mathrm{H}_{16} \mathrm{NO}$ $[\mathrm{M}+\mathrm{H}]^{+}$262.1226, Found 262.1230.<smiles>O[C@@H]1c2ccccc2N[C@H]1c1cccs1</smiles>

2-(thiophen-2-yl)indolin-3-ol (2o); Yellow solid was obtained in 60\% (65 mg) isolated yield; mp $81-84^{\circ} \mathrm{C}$; IR (KBr) v: 3367, 1315, 1241, 839, 752, 702; ${ }^{1} \mathrm{H}$ NMR $\left(400 \mathrm{MHz}, \mathrm{CDCl}_{3}\right) \delta 7.28(\mathrm{~d}, J=7.4$ $\mathrm{Hz}, 1 \mathrm{H}), 7.20-7.15(\mathrm{~m}, 2 \mathrm{H}), 7.03(\mathrm{~d}, J=3.3 \mathrm{~Hz}, 1 \mathrm{H}), 6.96-6.94(\mathrm{~m}, 1 \mathrm{H}), 6.83(\mathrm{t}, J=7.4 \mathrm{~Hz}, 1 \mathrm{H})$, $6.71(\mathrm{~d}, J=7.9 \mathrm{~Hz}, 1 \mathrm{H}), 5.08(\mathrm{~d}, J=5.2 \mathrm{~Hz}, 1 \mathrm{H}), 4.86(\mathrm{~d}, J=5.2 \mathrm{~Hz}, 1 \mathrm{H}), 4.27(\mathrm{br}, 1 \mathrm{H}), 2.50(\mathrm{br}, 1 \mathrm{H})$; ${ }^{13} \mathrm{C}$ NMR $\left(101 \mathrm{MHz}, \mathrm{CDCl}_{3}\right) \delta 149.55,145.91,129.94,128.94,126.96,125.17,124.46,124.14,119.78$, 110.31, 81.36, 68.34; HRMS (ESI) $m / z$ calcd for $\mathrm{C}_{12} \mathrm{H}_{11} \mathrm{NNaOS}[\mathrm{M}+\mathrm{Na}]^{+} 240.0454$, Found 240.0452.<smiles>[Y7][C@H]1Nc2ccccc2[C@H]1O</smiles>

2-ethylindolin-3-ol (2p); Yellow solid was obtained in $30 \%(37 \mathrm{mg})$ isolated yield; $\mathrm{mp} 39-42^{\circ} \mathrm{C}$; IR $(\mathrm{KBr})$ v: 3450, 1309, 1176, 915, 744; ${ }^{1} \mathrm{H} \mathrm{NMR}\left(400 \mathrm{MHz}, \mathrm{CDCl}_{3}\right) \delta 7.35-7.26(\mathrm{~m}, 1 \mathrm{H}), 7.16(\mathrm{t}, J=$ $7.6 \mathrm{~Hz}, 1 \mathrm{H}), 6.79(\mathrm{t}, J=7.4 \mathrm{~Hz}, 1 \mathrm{H}), 6.67(\mathrm{~d}, J=7.9 \mathrm{~Hz}, 1 \mathrm{H}), 4.88(\mathrm{~d}, J=4.0 \mathrm{~Hz}, 1 \mathrm{H}), 3.57(\mathrm{~d}, J=5.7$ $\mathrm{Hz}, 1 \mathrm{H}), 1.68-1.16(\mathrm{~m}, 14 \mathrm{H}), 0.91(\mathrm{t}, J=6.4 \mathrm{~Hz}, 3 \mathrm{H}) ;{ }^{13} \mathrm{C} \mathrm{NMR}\left(101 \mathrm{MHz}, \mathrm{CDCl}_{3}\right) \delta 150.15,129.77$, $125.22,118.87,110.18,78.58,68.55,34.70,31.88,29.65,29.55,29.27,26.24,22.68,14.12 ;$ HRMS (ESI) $m / z$ calcd for $\mathrm{C}_{16} \mathrm{H}_{26} \mathrm{NO}[\mathrm{M}+\mathrm{H}]^{+} 248.2009$, Found 248.2010.<smiles>CC(C)(C)[C@H]1Nc2ccccc2[C@@H]1O</smiles>

2-(tert-butyl)indolin-3-ol (2q); Yellow solid was obtained in 25\% (24 mg) isolated yield; mp 50-52 ${ }^{\circ}$; IR (KBr) v: 3425, 1358, 1291, 785, 744, 695; ${ }^{1} \mathrm{H} \mathrm{MR}\left(400 \mathrm{MHz}, \mathrm{CDCl}_{3}\right) \delta 7.28(\mathrm{~d}, J=7.4 \mathrm{~Hz}, 1 \mathrm{H})$, $7.12(\mathrm{t}, J=7.6 \mathrm{~Hz}, 1 \mathrm{H}), 6.73(\mathrm{t}, J=7.4 \mathrm{~Hz}, 1 \mathrm{H}), 6.63(\mathrm{~d}, J=7.9 \mathrm{~Hz}, 1 \mathrm{H}), 5.05(\mathrm{~d}, J=5.2 \mathrm{~Hz}, 1 \mathrm{H})$, $3.31(\mathrm{~d}, J=5.2 \mathrm{~Hz}, 1 \mathrm{H}), 0.95(\mathrm{~s}, 9 \mathrm{H}) ;{ }^{13} \mathrm{C} \mathrm{NMR}\left(101 \mathrm{MHz}, \mathrm{CDCl}_{3}\right) \delta 150.52,129.95,129.69,125.02$, 118.33, 109.44, 77.88, 74.71, 33.47, 25.82; HRMS (ESI) $m / z$ calcd for C12H18NO [M+H $]^{+} 192.1383$, Found192.1376. 
<smiles>Cc1ccc([C@@H]2Nc3cc(Cl)ccc3[C@H]2O)cc1</smiles>

6-chloro-2-(p-tolyl)indolin-3-ol (2r); Yellow solid was obtained in 49\% (63 mg) isolated yield; mp 190-191 ${ }^{\circ}$; IR (KBr) v: 3447, 1325, 1178, 807; ${ }^{1} \mathrm{H}$ NMR (400 MHz, CD $\left.{ }_{3} \mathrm{OD}\right) \delta 7.22(\mathrm{~d}, J=7.8 \mathrm{~Hz}, 2 \mathrm{H})$, $7.10(\mathrm{~d}, J=8.1 \mathrm{~Hz}, 3 \mathrm{H}), 6.64(\mathrm{~d}, J=5.8 \mathrm{~Hz}, 2 \mathrm{H}), 4.86(\mathrm{~d}, J=5.5 \mathrm{~Hz}, 1 \mathrm{H}), 4.57(\mathrm{~d}, J=5.4 \mathrm{~Hz}, 1 \mathrm{H})$, $2.28(\mathrm{~s}, 3 \mathrm{H}) ;{ }^{13} \mathrm{C}$ NMR $\left(101 \mathrm{MHz}, \mathrm{CD}_{3} \mathrm{OD}\right) \delta 152.53,139.54,136.81,134.72,128.80,127.91,125.87$, 125.63, 117.39, 108.75, 80.17, 71.97, 19.80; HRMS (ESI) $\mathrm{m} / z$ calcd for $\mathrm{C}_{15} \mathrm{H}_{14} \mathrm{ClNNaO}[\mathrm{M}+\mathrm{Na}]^{+}$ 282.0656, Found 282.0663.<smiles>Cc1ccc([C@@H]2Nc3cc(C)ccc3[C@@H]2O)cc1</smiles>

6-methyl-2-(p-tolyl)indolin-3-ol (2s); Yellow solid was obtained in 40\% (48 mg) isolated yield; mp $119-121^{\circ} \mathrm{C} ; \quad \operatorname{IR}(\mathrm{KBr}) v: 3445,1342,1305,849,800,736 ;{ }^{1} \mathrm{H}$ NMR $\left(400 \mathrm{MHz}, \mathrm{CDCl}_{3}\right) \delta 7.22(\mathrm{~d}, J=$ $7.9 \mathrm{~Hz}, 2 \mathrm{H}), 7.13(\mathrm{dd}, J=16.3,7.6 \mathrm{~Hz}, 3 \mathrm{H}), 6.62(\mathrm{~d}, J=7.5 \mathrm{~Hz}, 1 \mathrm{H}), 6.54(\mathrm{~s}, 1 \mathrm{H}), 4.97(\mathrm{~d}, J=4.4 \mathrm{~Hz}$, 1H), $4.59(\mathrm{~d}, J=4.4 \mathrm{~Hz}, 1 \mathrm{H}), 4.14(\mathrm{~s}, 1 \mathrm{H}), 3.44(\mathrm{~s}, 1 \mathrm{H}), 2.32(\mathrm{~s}, 3 \mathrm{H}), 2.31(\mathrm{~s}, 3 \mathrm{H}) ;{ }^{13} \mathrm{C} \mathrm{NMR}(101 \mathrm{MHz}$, $\left.\mathrm{CDCl}_{3}\right) \delta 150.82,140.13,139.40,137.34,129.38,126.28,126.16,125.05,120.11,110.47,81.16,72.29$ 21.71, 21.09; HRMS (ESI) $m / z$ calcd for $\mathrm{C}_{16} \mathrm{H}_{18} \mathrm{NO}[\mathrm{M}+\mathrm{H}]^{+} 240.1383$, Found 240.1383.<smiles>Cc1ccc([C@@H]2Nc3ccc(F)cc3[C@H]2O)cc1</smiles>

5-fluoro-2-(p-tolyl)indolin-3-ol (2t); Yellow solid was obtained in 62\% (75 mg) isolated yield; mp 110-113을 IR (KBr) v: 3444, 1392, 1172, 754; ${ }^{1} \mathrm{H}$ NMR $\left(400 \mathrm{MHz}, \mathrm{CDCl}_{3}\right) \delta 7.23(\mathrm{~d}, J=8.2 \mathrm{~Hz}, 2 \mathrm{H})$, $7.13(\mathrm{~d}, J=7.8 \mathrm{~Hz}, 2 \mathrm{H}), 6.97(\mathrm{dd}, J=7.9,1.9 \mathrm{~Hz}, 1 \mathrm{H}), 6.87(\mathrm{td}, J=8.9,2.3 \mathrm{~Hz}, 1 \mathrm{H}), 6.61(\mathrm{dd}, J=8.5$, $4.1 \mathrm{~Hz}, 1 \mathrm{H}), 4.97(\mathrm{~d}, J=5.5 \mathrm{~Hz}, 1 \mathrm{H}), 4.60(\mathrm{~d}, J=5.5 \mathrm{~Hz}, 1 \mathrm{H}), 3.02(\mathrm{~s}, 2 \mathrm{H}), 2.33(\mathrm{~s}, 3 \mathrm{H}) ;{ }^{13} \mathrm{C} \mathrm{NMR}$ $\left(101 \mathrm{MHz}, \mathrm{CDCl}_{3}\right) \delta 158.39,156.04,146.33,138.81,137.60,130.55,129.44,126.24,116.29,116.06$, 112.32, 112.08, 110.37, 110.29, 81.20, 72.86, 21.10; HRMS (ESI) $m / z$ calcd for $\mathrm{C}_{15} \mathrm{H}_{15} \mathrm{FNO}[\mathrm{M}+\mathrm{H}]^{+}$ 244.1132, Found 244.1134.<smiles>COc1cccc([C@@H]2Nc3cc(C)ccc3[C@H]2O)c1</smiles> 
2-(3-methoxyphenyl)-6-methylindolin-3-ol (2u); Yellow solid was obtained in $52 \%$ (66 mg) isolated yield; mp $121-123^{\circ} \mathrm{C}$; IR (KBr) v: $3452,1454,1257,849,781,695 ;{ }^{1} \mathrm{H}$ NMR (400 MHz, DMSO) $\delta$ $7.25(\mathrm{t}, J=7.8 \mathrm{~Hz}, 1 \mathrm{H}), 7.01-6.93(\mathrm{~m}, 3 \mathrm{H}), 6.83(\mathrm{~d}, J=8.1 \mathrm{~Hz}, 1 \mathrm{H}), 6.45(\mathrm{~d}, J=7.9 \mathrm{~Hz}, 2 \mathrm{H}), 6.02(\mathrm{~s}$, $1 \mathrm{H}), 5.60(\mathrm{~d}, J=6.3 \mathrm{~Hz}, 1 \mathrm{H}), 4.77(\mathrm{t}, J=5.9 \mathrm{~Hz}, 1 \mathrm{H}), 4.48(\mathrm{br}, 1 \mathrm{H}), 3.74(\mathrm{~s}, 3 \mathrm{H}), 2.22(\mathrm{~s}, 3 \mathrm{H}) ;{ }^{13} \mathrm{C}$ NMR (101 MHz, DMSO) $\delta$ 159.76, 151.73, 145.85, 138.38, 129.81, 127.78, 124.86, 118.99, 118.45, $112.75,112.33,109.86,80.56,72.02,55.43,21.82$; HRMS (ESI) $m / z$ calcd for $\mathrm{C}_{16} \mathrm{H}_{18} \mathrm{NO}_{2}[\mathrm{M}+\mathrm{H}]^{+}$ 256.1332, Found 256.1331.<smiles>O[C@@H]1c2ccc(Cl)cc2N[C@H]1c1ccc(Cl)cc1</smiles>

6-chloro-2-(4-chlorophenyl)indolin-3-ol (2v); Yellow solid was obtained in 53\% (74 mg) isolated yield; mp 82.2-84.2 ${ }^{\circ} \mathrm{C}$; IR (KBr) v: 3426, 1483, 1310, 911, 825; ${ }^{1} \mathrm{H}$ NMR $\left(400 \mathrm{MHz}, \mathrm{CDCl}_{3}\right) \delta 7.26(\mathrm{q}$, $J=8.2 \mathrm{~Hz}, 4 \mathrm{H}), 7.12(\mathrm{~d}, J=7.9 \mathrm{~Hz}, 1 \mathrm{H}), 6.74(\mathrm{~d}, J=7.9 \mathrm{~Hz}, 1 \mathrm{H}), 6.66(\mathrm{~s}, 1 \mathrm{H}), 4.88(\mathrm{~d}, J=4.2 \mathrm{~Hz}$, 1H), $4.58(\mathrm{~d}, J=4.4 \mathrm{~Hz}, 1 \mathrm{H}), 4.25$ (br, 1H). 2.79 (br, $1 \mathrm{H}) ;{ }^{13} \mathrm{C} \mathrm{NMR}\left(101 \mathrm{MHz}, \mathrm{CDCl}_{3}\right) \delta 151.25$, $140.18,135.74,133.61,128.96,127.56,127.13,126.13,119.35,109.92,80.53,71.94$; HRMS (ESI) $m / z$ calcd for $\mathrm{C}_{14} \mathrm{H}_{12} \mathrm{Cl}_{2} \mathrm{NO}[\mathrm{M}+\mathrm{H}]^{+} 280.0290$, Found 280.0289<smiles>Cc1ccc2c(c1)N[C@H](c1ccc(Cl)cc1)[C@H]2O</smiles>

2-(4-chlorophenyl)-6-methylindolin-3-ol (2w); Yellow solid was obtained in 70\% (90 mg) isolated yield; mp 100-102 ${ }^{\circ}$; IR (KBr) v: 3423, 1455, 1266, 810, 740; ${ }^{1} \mathrm{H}$ NMR (400 MHz, $\left.\mathrm{CDCl}_{3}\right) \delta 7.31-$ $7.26(\mathrm{~m}, 4 \mathrm{H}), 7.16(\mathrm{~d}, J=7.5 \mathrm{~Hz}, 1 \mathrm{H}), 6.64(\mathrm{~d}, J=7.5 \mathrm{~Hz}, 1 \mathrm{H}), 6.56(\mathrm{~s}, 1 \mathrm{H}), 4.94(\mathrm{~d}, J=4.5 \mathrm{~Hz}, 1 \mathrm{H})$, $4.61(\mathrm{~d}, J=4.6 \mathrm{~Hz}, 1 \mathrm{H}), 4.13(\mathrm{br}, 1 \mathrm{H}), 2.31(\mathrm{~s}, 3 \mathrm{H}) ;{ }^{13} \mathrm{C} \mathrm{NMR}\left(101 \mathrm{MHz}, \mathrm{CDCl}_{3}\right) \delta 150.49,140.93$, 140.34, 133.33, 128.83, 127.62, 125.94, 124.99, 120.39, 110.58, 81.19, 71.90, 21.71; HRMS (ESI) $\mathrm{m} / \mathrm{z}$ calcd for $\mathrm{C}_{15} \mathrm{H}_{15} \mathrm{ClNO}[\mathrm{M}+\mathrm{H}]^{+} 260.0837$, Found 260.0840 .

\section{Characterization Data for 3}<smiles>Cc1ccc(C2=Nc3ccccc3C2=O)cc1</smiles>

2-(p-tolyl)-3H-indol-3-one (3a); Red solid was obtained in $46 \%(51 \mathrm{mg})$ isolated yield; $\mathrm{mp} 79^{\circ} \mathrm{C}$; IR (KBr) v: 1721, 1536, 1275, 875, 752; ${ }^{1} \mathrm{H}$ NMR (400 MHz, $\left.\mathrm{CDCl}_{3}\right) \delta 8.28(\mathrm{~d}, J=8.2 \mathrm{~Hz}, 2 \mathrm{H}), 7.52(\mathrm{t}, J$ 
$=7.8 \mathrm{~Hz}, 2 \mathrm{H}), 7.39(\mathrm{~d}, J=7.4 \mathrm{~Hz}, 1 \mathrm{H}), 7.29(\mathrm{~d}, J=8.1 \mathrm{~Hz}, 2 \mathrm{H}), 7.23(\mathrm{t}, J=7.4 \mathrm{~Hz}, 1 \mathrm{H}), 2.42(\mathrm{~s}, 3 \mathrm{H})$; ${ }^{13} \mathrm{C}$ NMR (101 MHz, $\left.\mathrm{CDCl}_{3}\right) \delta 193.89,161.05,160.08,142.95,136.75,129.63,129.29,128.05,127.31$, 124.62, 123.23, 121.79, 21.78; HRMS (ESI) $\mathrm{m} / \mathrm{z}$ calcd for $\mathrm{C}_{15} \mathrm{H}_{12} \mathrm{NO}[\mathrm{M}+\mathrm{H}]^{+} 222.0913$, Found 222.0912.<smiles>COc1ccc(C2=Nc3ccccc3C2=O)cc1</smiles>

2-(4-methoxyphenyl)-3H-indol-3-one (3c); Red solid was obtained in 64\% (76 mg) isolated yield; mp 104.9-105.8 ${ }^{\circ}$; IR (KBr) v: 1754, 1601, 1257, 839, 759; ${ }^{1} \mathrm{H}$ NMR (400 MHz, $\left.\mathrm{CDCl}_{3}\right) \delta 8.37(\mathrm{~d}, J=8.7$ $\mathrm{Hz}, 2 \mathrm{H}), 7.49(\mathrm{t}, J=7.1 \mathrm{~Hz}, 2 \mathrm{H}), 7.33(\mathrm{~d}, J=7.5 \mathrm{~Hz}, 1 \mathrm{H}), 7.18(\mathrm{t}, J=7.4 \mathrm{~Hz}, 1 \mathrm{H}), 6.97(\mathrm{~d}, J=8.7 \mathrm{~Hz}$, 2H), $3.86(\mathrm{~s}, 3 \mathrm{H}) ;{ }^{13} \mathrm{C}$ NMR (101 MHz, $\left.\mathrm{CDCl}_{3}\right) \delta 194.27,163.03,160.34,160.27,136.78,131.28$, 127.67, 124.58, 123.22, 122.69, 121.51, 114.41, 55.43; HRMS (ESI) $\mathrm{m} / z$ calcd for $\mathrm{C}_{15} \mathrm{H}_{12} \mathrm{NO}_{2}[\mathrm{M}+\mathrm{H}]^{+}$ 238.0863, Found 238.0864.<smiles>CCc1ccc(C2=Nc3ccccc3C2=O)cc1</smiles>

2-(4-ethylphenyl)-3H-indol-3-one(3e); Red solid was obtained in 45\% (53 mg) isolated yield; $\mathrm{mp}$ 54.1-55.9 ${ }^{\circ} \mathrm{C}$; IR (KBr) v: 1702, 1492, 1275, 841, 754; ${ }^{1} \mathrm{H}$ NMR (400 MHz, $\left.\mathrm{CDCl}_{3}\right) \delta 8.31(\mathrm{~d}, J=7.8$ $\mathrm{Hz}, 2 \mathrm{H}), 7.53(\mathrm{t}, J=8.0 \mathrm{~Hz}, 2 \mathrm{H}), 7.39(\mathrm{~d}, J=7.4 \mathrm{~Hz}, 1 \mathrm{H}), 7.32(\mathrm{~d}, J=7.8 \mathrm{~Hz}, 2 \mathrm{H}), 7.26-7.22(\mathrm{~m}$, 1H), $2.72(\mathrm{q}, J=7.6 \mathrm{~Hz}, 2 \mathrm{H}), 1.28(\mathrm{t}, J=7.5 \mathrm{~Hz}, 3 \mathrm{H}) ;{ }^{13} \mathrm{C} \mathrm{NMR}\left(101 \mathrm{MHz}, \mathrm{CDCl}_{3}\right) \delta$ 193.92, 161.07, $160.06,149.18,136.79,129.41,128.45,128.08,127.48,124.65,123.21,121.80,29.07,15.19 ;$ HRMS (ESI) $\mathrm{m} / \mathrm{z}$ calcd for $\mathrm{C}_{16} \mathrm{H}_{14} \mathrm{NO}[\mathrm{M}+\mathrm{H}]^{+} 236.1072$, Found 236.1070 .<smiles>COc1cccc(C2=Nc3ccccc3C2=O)c1</smiles>

2-(3-methoxyphenyl)-3H-indol-3-one (3f); Red syrup was obtained in 43\% (51 mg) isolated yield; IR (KBr) v: 1723, 1599, 1266, 865, 753; ${ }^{1} \mathrm{H}$ NMR $\left(400 \mathrm{MHz}, \mathrm{CDCl}_{3}\right) \delta 8.00(\mathrm{~d}, J=7.7 \mathrm{~Hz}, 1 \mathrm{H}), 7.90(\mathrm{~s}$, 1H), $7.54(\mathrm{t}, J=7.4 \mathrm{~Hz}, 2 \mathrm{H}), 7.44-7.37(\mathrm{~m}, 2 \mathrm{H}), 7.26(\mathrm{t}, J=7.3 \mathrm{~Hz}, 1 \mathrm{H}), 7.09(\mathrm{~d}, J=8.2 \mathrm{~Hz}, 1 \mathrm{H})$, 3.90 (s, 3H); ${ }^{13} \mathrm{C}$ NMR $\left(101 \mathrm{MHz}, \mathrm{CDCl}_{3}\right) \delta 193.39,161.02,159.80,159.67,136.78,131.24,129.82$, $128.39,124.70,123.19,122.17,122.00,119.17,113.00,55.46$; HRMS (ESI) $\mathrm{m} / \mathrm{z}$ calcd for C15H12NO2 [M+H] $]^{+} 238.0863$, Found 238.0862. 
<smiles></smiles>

2-(o-tolyl)-3H-indol-3-one (3h); Red solid was obtained in 62\% (68 mg) isolated yield; $\mathrm{mp} 71.0$ $-72.3^{\circ} \mathrm{C}$; IR (KBr) v: 1724, 1599, 1215, 882, 796; ${ }^{1} \mathrm{H}$ NMR (400 MHz, $\left.\mathrm{CDCl}_{3}\right) \delta 7.74$ (d, $J=7.6 \mathrm{~Hz}$, 1H), $7.50-7.45(\mathrm{~m}, 2 \mathrm{H}), 7.37-7.29(\mathrm{~m}, 2 \mathrm{H}), 7.26-7.17$ (m, 3H), 2.55 (s, 3H); ${ }^{13} \mathrm{C}$ NMR $(101 \mathrm{MHz}$, $\left.\mathrm{CDCl}_{3}\right) \delta 193.33,164.20,160.16,139.71,136.73,131.56,131.21,130.77,128.71,128.43,125.71$, 124.77, 122.12, 122.09, 21.75; HRMS (ESI) m/z calcd for $\mathrm{C}_{15} \mathrm{H}_{12} \mathrm{NO}[\mathrm{M}+\mathrm{H}]^{+} 222.0913$, Found 222.0914 .<smiles>O=C1C(c2cccc3ccccc23)=Nc2ccccc21</smiles>

2-(naphthalen-1-yl)-3H-indol-3-one (3m); Red syrup was obtained in 68\% (87 mg) isolated yield; IR (KBr) v: 1718, 1595, 1284, 878, 762; ${ }^{1} \mathrm{H}$ NMR $\left(400 \mathrm{MHz}, \mathrm{CDCl}_{3}\right) \delta 9.00(\mathrm{~d}, J=8.6 \mathrm{~Hz}, 1 \mathrm{H}), 8.13(\mathrm{~d}, J$ $=7.3 \mathrm{~Hz}, 1 \mathrm{H}), 7.92(\mathrm{~d}, J=8.2 \mathrm{~Hz}, 1 \mathrm{H}), 7.83(\mathrm{~d}, J=8.1 \mathrm{~Hz}, 1 \mathrm{H}), 7.58-7.45(\mathrm{~m}, 6 \mathrm{H}), 7.23(\mathrm{t}, J=7.3$ $\mathrm{Hz}, 1 \mathrm{H}) ;{ }^{13} \mathrm{C}$ NMR $\left(101 \mathrm{MHz}, \mathrm{CDCl}_{3}\right) \delta 193.63,162.61,160.30,136.89,134.12,132.38,131.83$, $131.73,128.81,128.48,127.72,126.49,126.40,125.90,124.90,122.26,122.08$; HRMS (ESI) $\mathrm{m} / z$ calcd for $\mathrm{C}_{18} \mathrm{H}_{12} \mathrm{NO}[\mathrm{M}+\mathrm{H}]^{+}$258.0913, Found 258.0914.<smiles>O=[N+]([O-])c1ccccc1/C=C/c1ccccc1</smiles>

(E)-1-nitro-2-styrylbenzene (4); ${ }^{1}$ Yellow solid was obtained in 72\% (590 mg) isolated yield; ${ }^{1} \mathrm{H}$ NMR $\left(400 \mathrm{MHz}, \mathrm{CDCl}_{3}\right) \delta 7.94(\mathrm{~d}, J=8.2 \mathrm{~Hz}, 1 \mathrm{H}), 7.75(\mathrm{~d}, J=7.9 \mathrm{~Hz}, 1 \mathrm{H}), 7.62-7.50(\mathrm{~m}, 4 \mathrm{H}), 7.40-$ $7.34(\mathrm{~m}, 3 \mathrm{H}), 7.31(\mathrm{~d}, J=7.3 \mathrm{~Hz}, 1 \mathrm{H}), 7.07(\mathrm{~d}, J=16.1 \mathrm{~Hz}, 1 \mathrm{H}) ;{ }^{13} \mathrm{C} \mathrm{NMR}\left(101 \mathrm{MHz}, \mathrm{CDCl}_{3}\right) \delta$ $148.04,136.53,133.89,133.10,133.03,128.84,128.64,128.18,127.98,127.12,124.77,123.51$.<smiles>O=C1C(c2ccccc2)=C([N+](=O)[O-])c2ccccc21</smiles>

3-oxo-2-phenyl-3H-indole 1-oxide (5b); ${ }^{2}$ Red solid was obtained in $60 \%(67 \mathrm{mg})$ isolated yield; ${ }^{1} \mathrm{H}$ $\operatorname{NMR}\left(400 \mathrm{MHz}, \mathrm{CDCl}_{3}\right) \delta 8.64(\mathrm{~d}, J=6.8 \mathrm{~Hz}, 2 \mathrm{H}), 7.72-7.66(\mathrm{~m}, 2 \mathrm{H}), 7.63(\mathrm{~s}, 1 \mathrm{H}), 7.56-7.46(\mathrm{~m}$, 
4H); ${ }^{13} \mathrm{C}$ NMR (101 MHz, $\left.\mathrm{CDCl}_{3}\right) \delta 186.96,147.87,134.85,131.21,130.76,129.75,128.57,127.87$, $125.86,122.82,121.64,114.22$.

1. Yang, K.; Zhou, F.; Kuang, Z.; Gao, G.; Driver, T. G.; Song, Q. Org. Lett. 2016, 18, 4088.

2. Ramana, C. V.; Patel, P.; Vanka, K.; Miao, B.; Degterev, A. Eur. J. Org. Chem. 2010, 2010, 5955.

\section{1 mmol scale reaction}

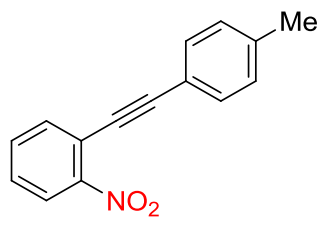

$1 a$

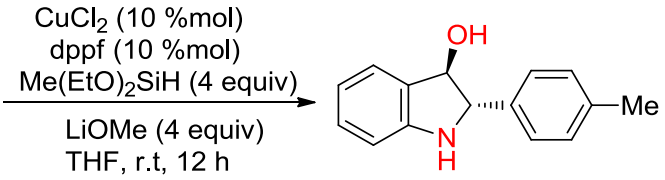

$2 a$

To a stirred solution of $\mathbf{1 a}(0.237 \mathrm{~g}, 1 \mathrm{mmol}$,$) in dry THF (3 \mathrm{~mL})$ were added $\mathrm{CuCl}_{2}(13.4 \mathrm{mg}, 0.1$ mmol, $10 \mathrm{~mol} \%)$, dppf (55.4 mg, $0.1 \mathrm{mmol}, 10 \mathrm{~mol} \%)$, LiOMe (152 mg, 4 mmol, 4 equiv) and $\mathrm{Me}(\mathrm{EtO})_{2} \mathrm{SiH}$ (537 mg, $4 \mathrm{mmol}, 4$ equiv) subsequently in a Schlenk flask under nitrogen atmosphere. After being stirred at room temperature for $12 \mathrm{~h}$, The resulting mixture was extracted with AcOEt $(3 \times 5$ $\mathrm{mL}$ ). The combined organic extracts were washed with brine, dried over $\mathrm{Na}_{2} \mathrm{SO}_{4}$, filtered and concentrated. The residue was purified by flash chromatography on a silica gel (using petroleum ether/ethyl acetate $=5: 1$ as the eluent $)$ to give corresponding product $\mathbf{2 a}$ in $56 \%$ yield $(0.125 \mathrm{~g})$. 
X-Ray crystal structure of 2a with 30\% ellipsoid probability (CCDC 1878765).

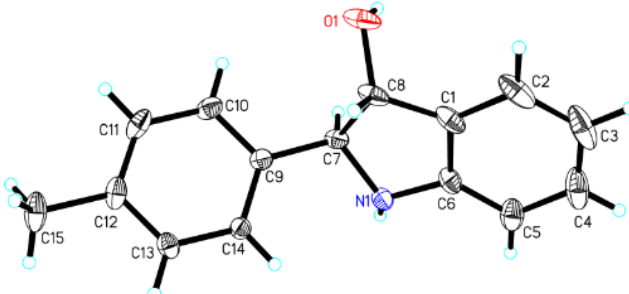<smiles>Cc1ccc(C2NC(=[Ge])c3ccccc32)cc1</smiles>

Table 1 Crystal data and structure refinement for $\mathrm{BH} 0816$.

Identification code

BH0816

Empirical formula

$\mathrm{C}_{15} \mathrm{H}_{15} \mathrm{NO}$

Formula weight

225.28

Temperature/K

120.01(10)

Crystal system

monoclinic

Space group

$\mathrm{P} 2{ }_{1} / \mathrm{c}$

$\mathrm{a} / \AA$

11.9454(8)

$\mathrm{b} / \AA ̊$

5.0007(3)

$\mathrm{c} / \AA$

20.0950(19)

$\alpha /{ }^{\circ}$

90

$\beta /{ }^{\circ}$

95.835(7)

$\gamma /{ }^{\circ}$

90

Volume $/ \AA^{3}$

1194.16(16)

Z

4

$\rho_{\text {calc }} / \mathrm{cm}^{3}$

1.253

$\mu / \mathrm{mm}^{-1}$

0.615

$\mathrm{F}(000)$

480.0

Crystal size $/ \mathrm{mm}^{3}$

$0.12 \times 0.11 \times 0.1$

Radiation

$\mathrm{CuK} \alpha(\lambda=1.54184)$

$2 \Theta$ range for data collection/ ${ }^{\circ} 7.44$ to 147.068

Index ranges

$-14 \leq \mathrm{h} \leq 14,-3 \leq \mathrm{k} \leq 5,-24 \leq 1 \leq 21$

Reflections collected

3955

Independent reflections

$2294\left[\mathrm{R}_{\text {int }}=0.0388, \mathrm{R}_{\text {sigma }}=0.0646\right]$

Data/restraints/parameters

$2294 / 0 / 156$

Goodness-of-fit on $\mathrm{F}^{2}$

1.031

Final R indexes $[\mathrm{I}>=2 \sigma(\mathrm{I})]$

$\mathrm{R}_{1}=0.0694, \mathrm{wR}_{2}=0.1798$

Final $\mathrm{R}$ indexes [all data]

$\mathrm{R}_{1}=0.0946, \mathrm{wR}_{2}=0.2114$

Largest diff. peak/hole / e $\AA^{-3} 0.29 /-0.30$ 
2-(p-tolyl)indolin-3-ol (2a)
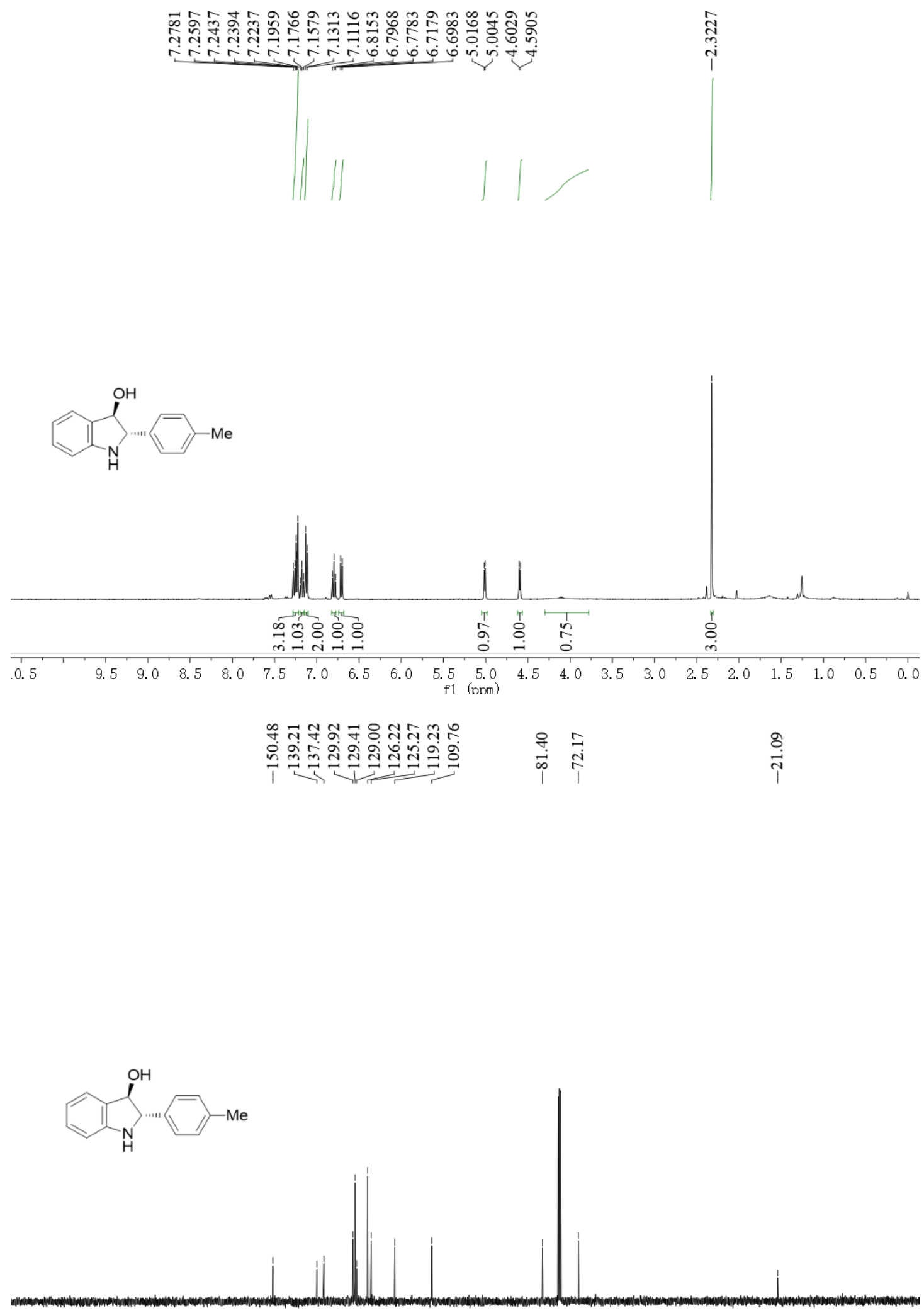

$\begin{array}{llllllllllllllllllllllllll}1 & 200 & 190 & 180 & 170 & 160 & 150 & 140 & 130 & 120 & 110 & 100 & 90 & 80 & 70 & 60 & 50 & 40 & 30 & 20 & 10 & 0 & -10\end{array}$ 


\section{2-phenylindolin-3-ol (2b)}
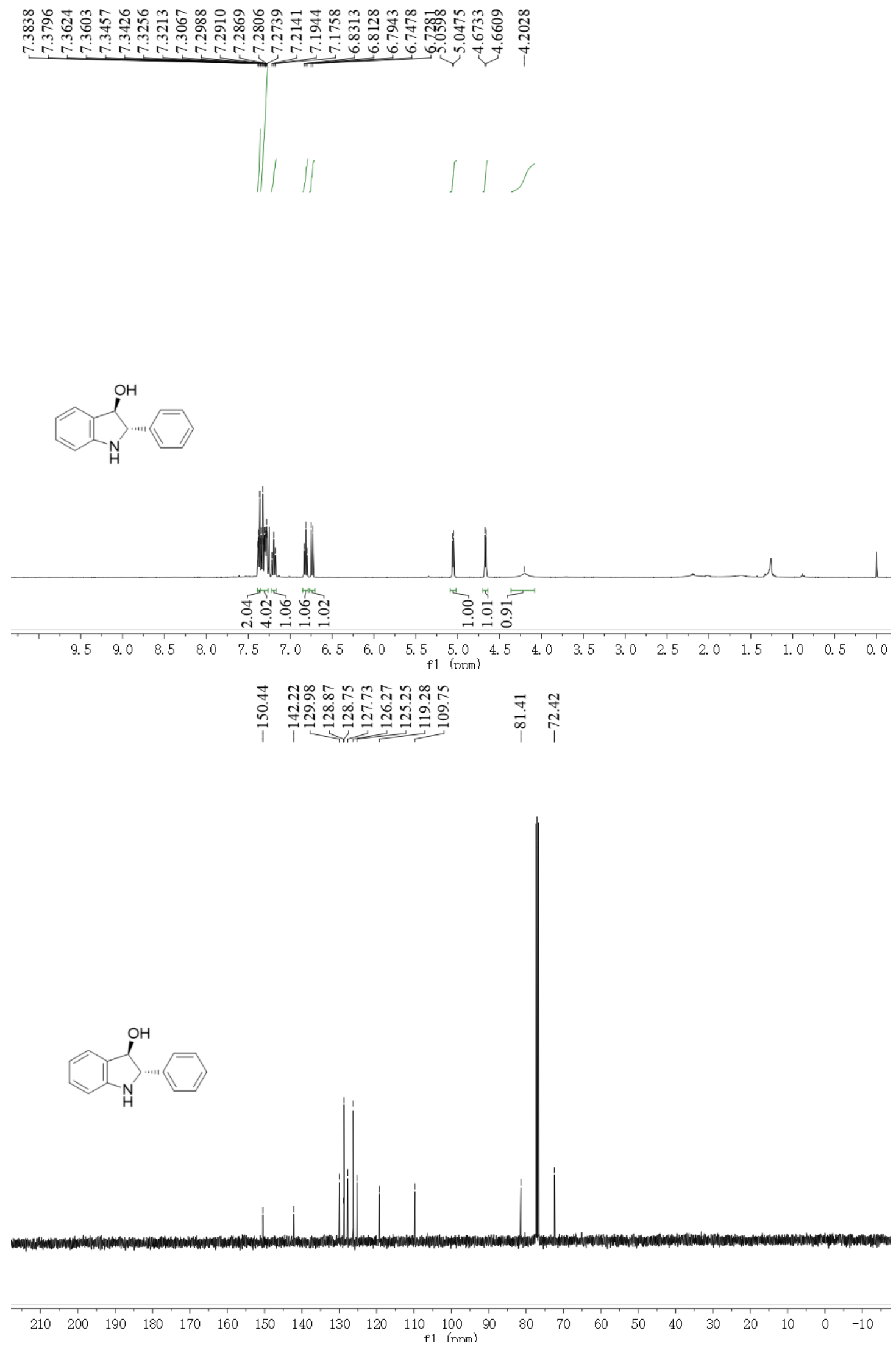


\section{2-(4-methoxyphenyl)indolin-3-ol (2c)}
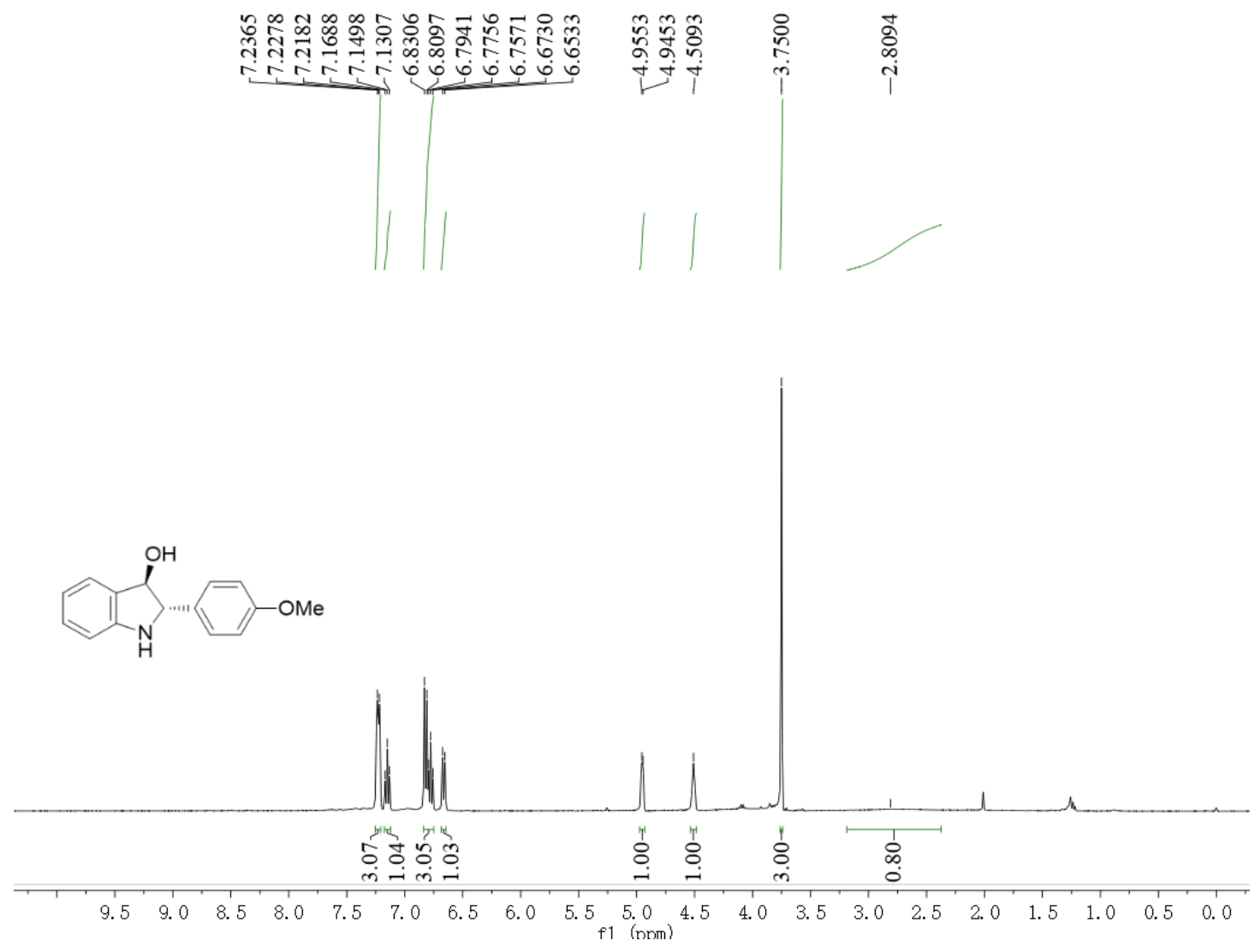

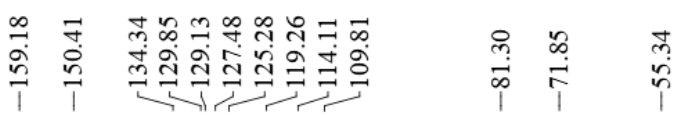
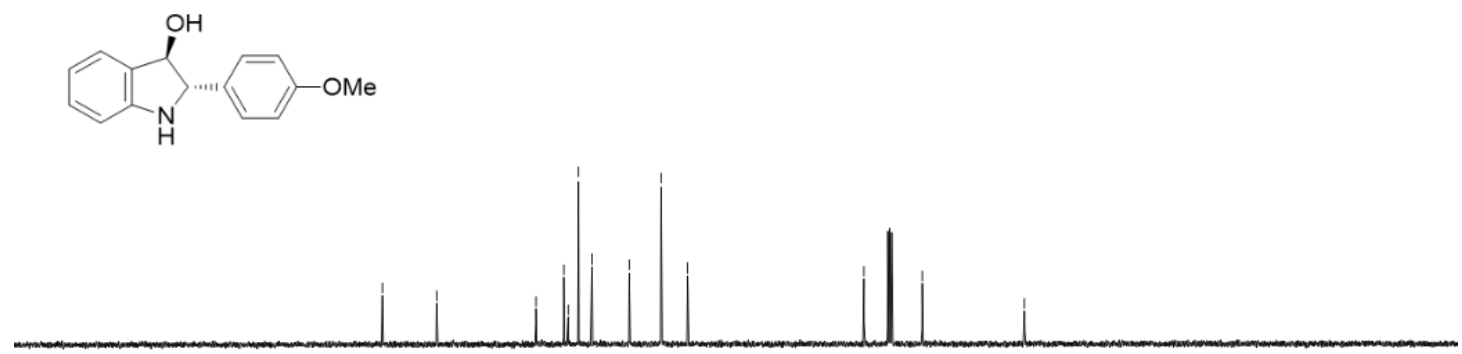

$\begin{array}{rllllllllllllllllllllllllllll}210 & 200 & 190 & 180 & 170 & 160 & 150 & 140 & 130 & 120 & 110 & 100 & 90 & 80 & 70 & 60 & 50 & 40 & 30 & 20 & 10 & 0 & -10\end{array}$ 
2-(4-(tert-butyl)phenyl)indolin-3-ol (2d)

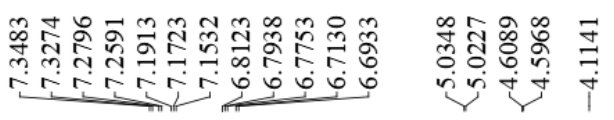

令

$\sum_{H}^{O H}-\cdots-t-B u$
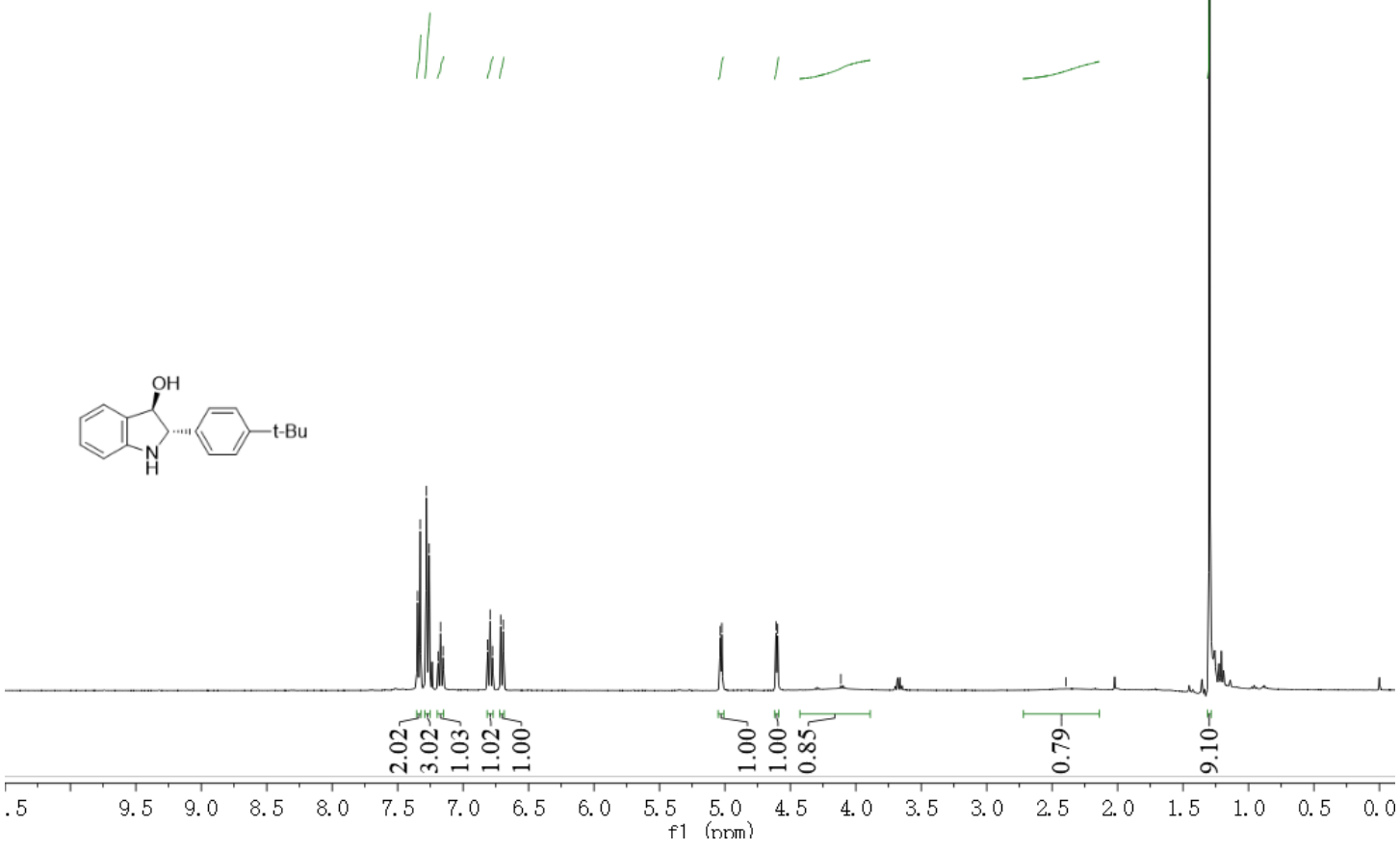

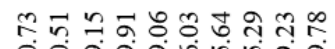

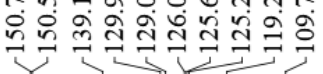

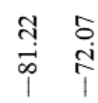

मेंक ले

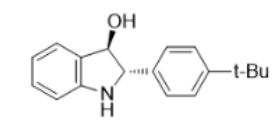

$\begin{array}{rlllllllllllllllllllllllllllll}210 & 200 & 190 & 180 & 170 & 160 & 150 & 140 & 130 & 120 & 110 & 100 & 90 & 80 & 70 & 60 & 50 & 40 & 30 & 20 & 10 & 0 & -10\end{array}$ 
2-(4-ethylphenyl)indolin-3-ol (2e)
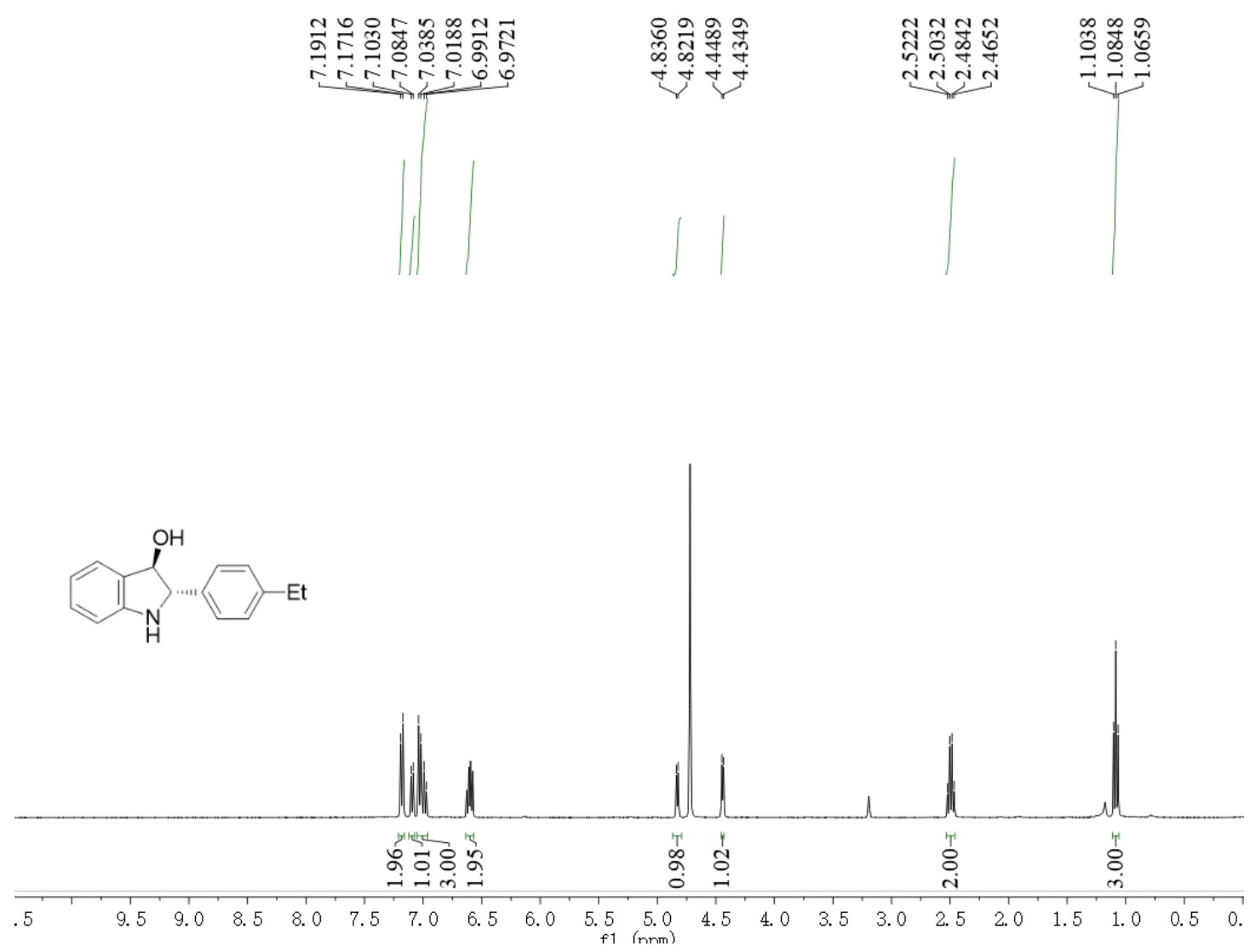

क्ष 90 욤

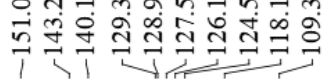

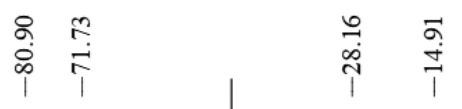

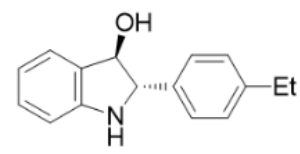

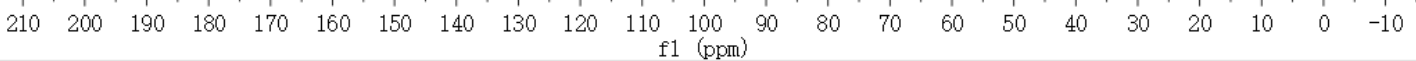


2-(3-methoxyphenyl)indolin-3-ol (2f)

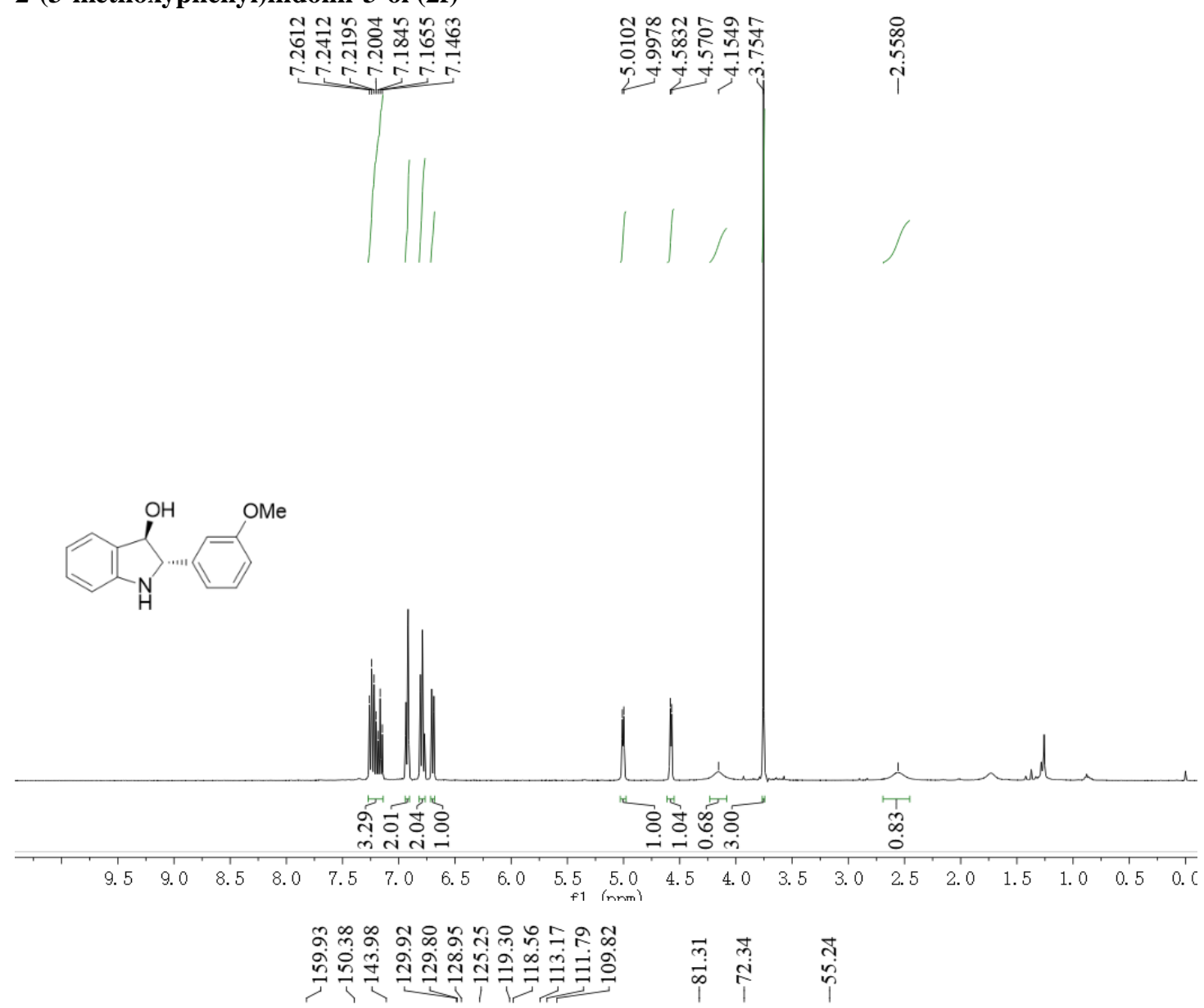<smiles>COc1cccc(C2Nc3ccccc3C2O)c1</smiles>

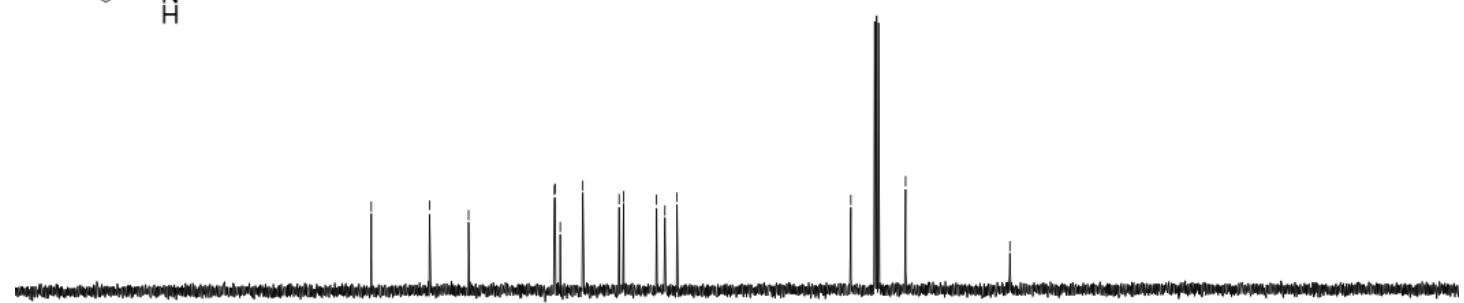

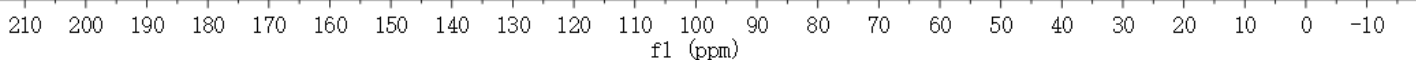




\section{2-(m-tolyl)indolin-3-ol (2g)}
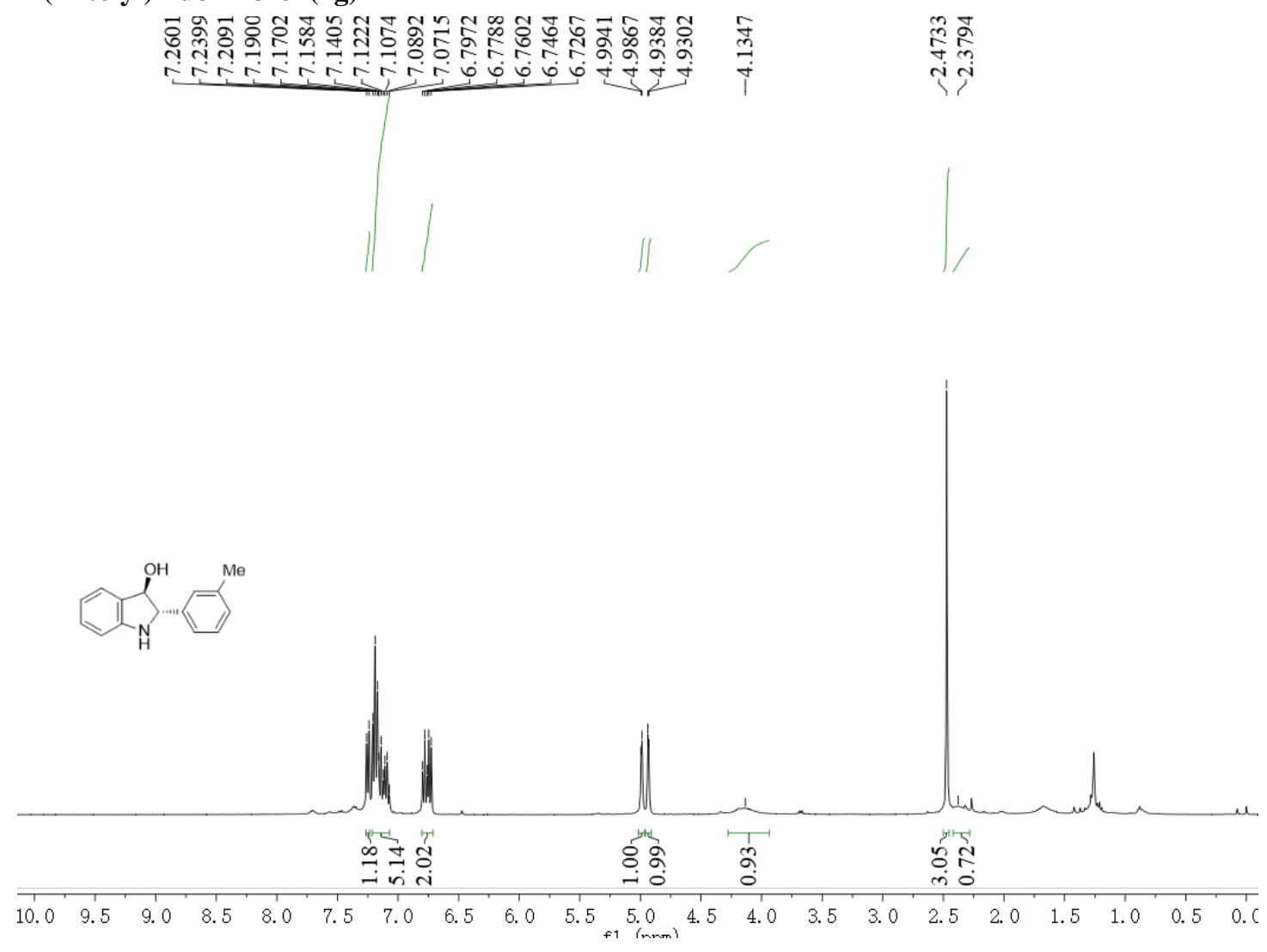

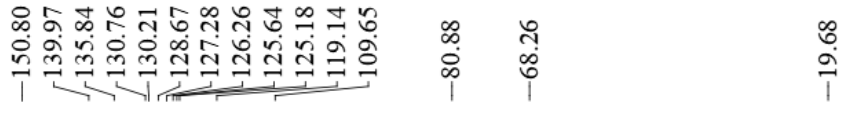
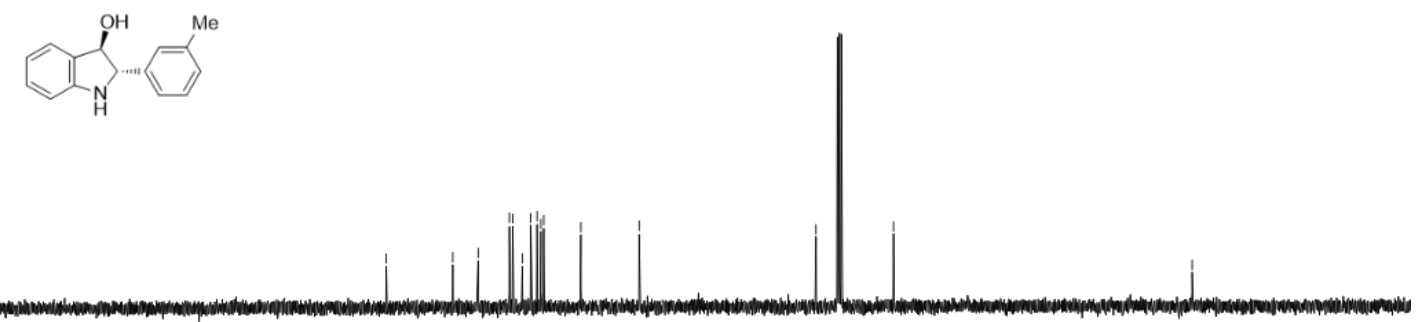

$\begin{array}{lllllllllllllllllllllll}210 & 200 & 190 & 180 & 170 & 160 & 150 & 140 & 130 & 120 & 110 & 100 & 90 & 80 & 70 & 60 & 50 & 40 & 30 & 20 & 10 & 0 & -10\end{array}$ 


\section{2-(o-tolyl)indolin-3-ol (2h)}

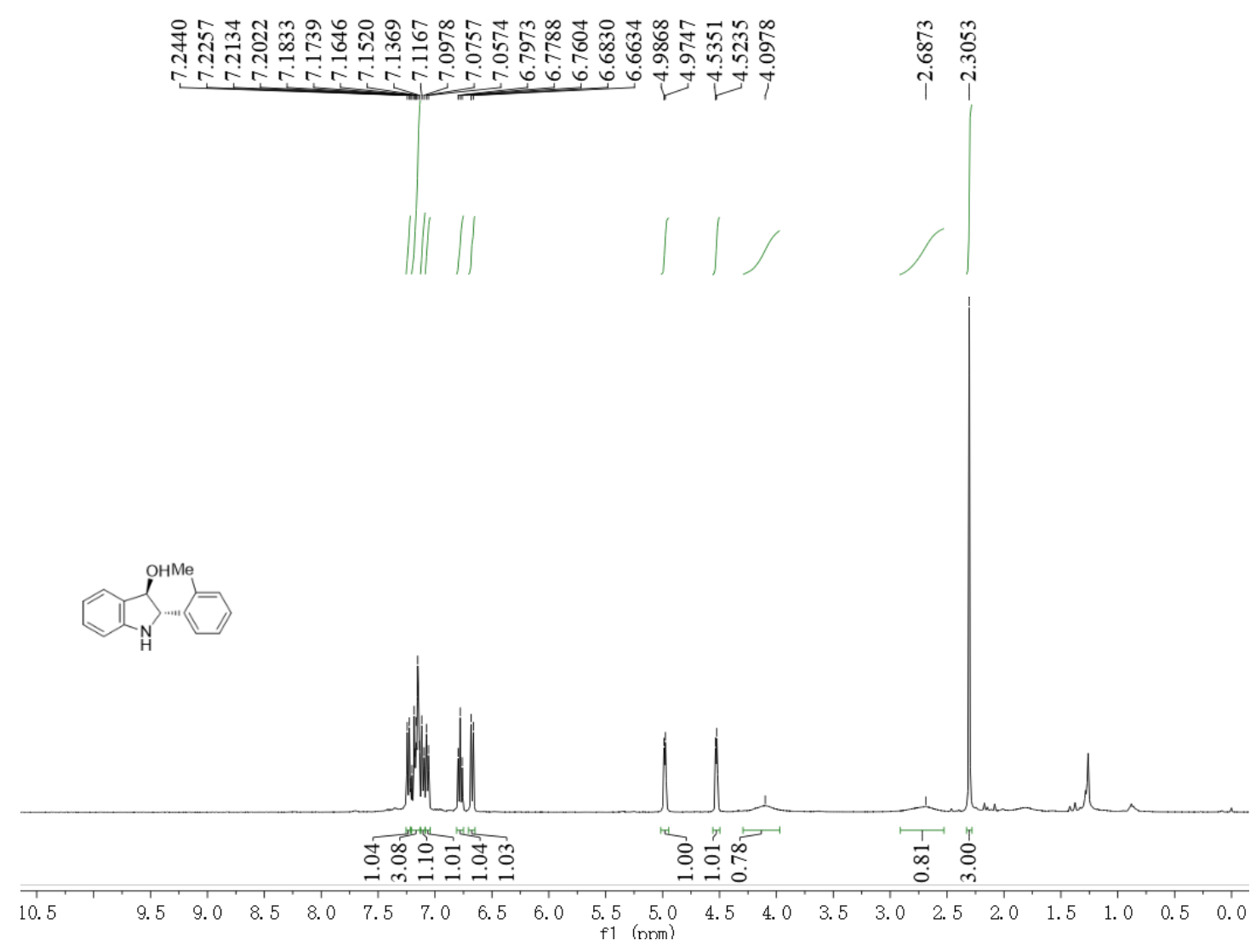

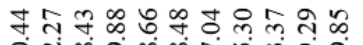

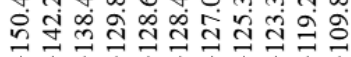

ॠ্

ฆั
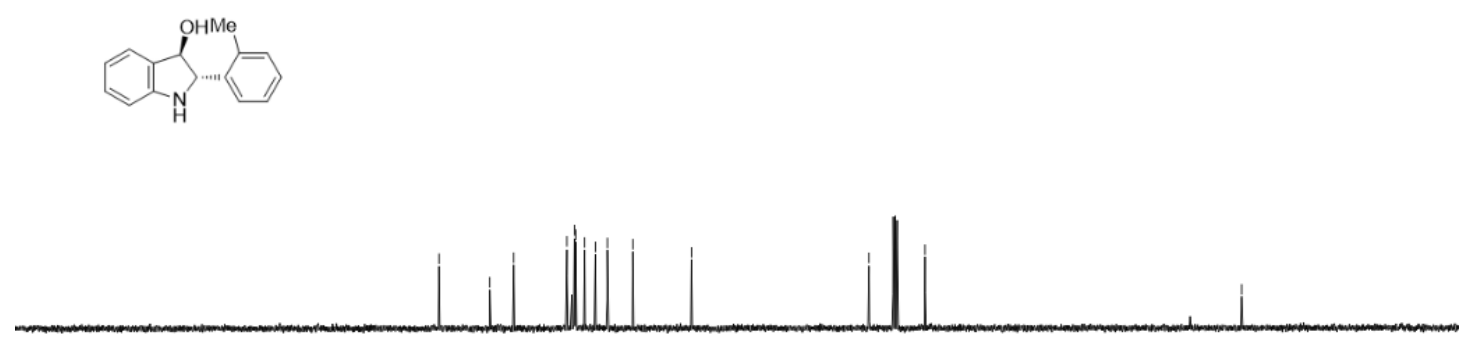

$\begin{array}{llllllllllllllllllllllllllllll}210 & 200 & 190 & 180 & 170 & 160 & 150 & 140 & 130 & 120 & 110 & 100 & 90 & 80 & 70 & 60 & 50 & 40 & 30 & 20 & 10 & 0 & -10\end{array}$ 
2-(4-bromophenyl)indolin-3-ol (2i)
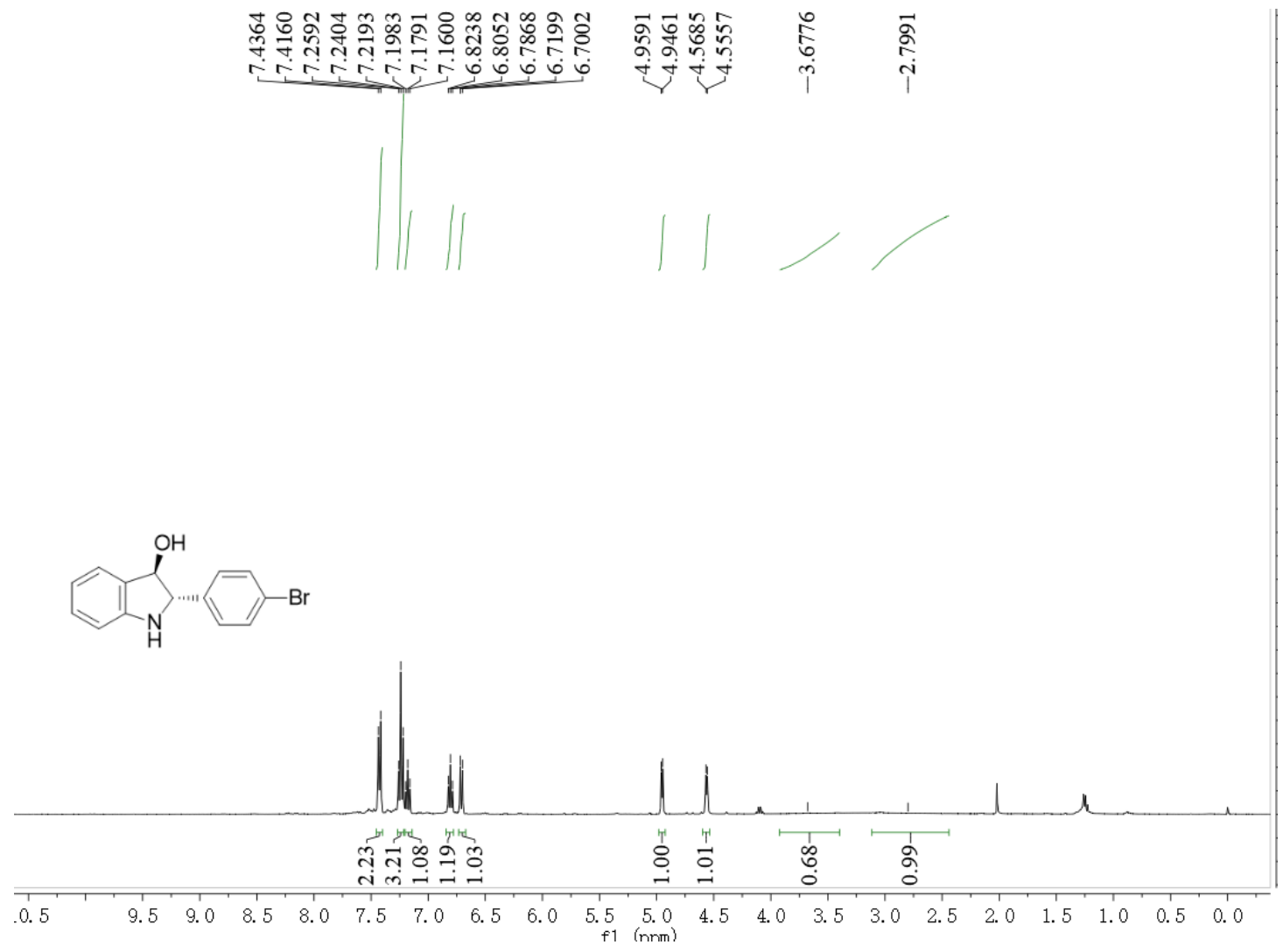

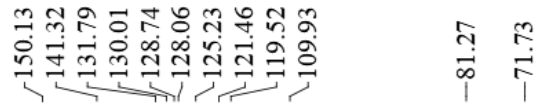
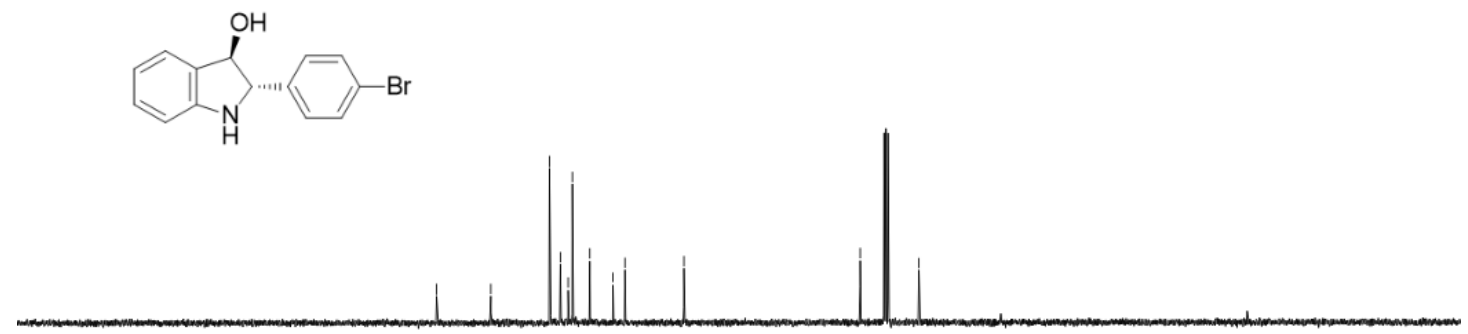

$\begin{array}{llllllllllllllllllllllll}210 & 200 & 190 & 180 & 170 & 160 & 150 & 140 & 130 & 120 & 110 & 100 & 90 & 80 & 70 & 60 & 50 & 40 & 30 & 20 & 10 & 0 & -10\end{array}$ 


\section{2-(4-fluorophenyl)indolin-3-ol (2j)}
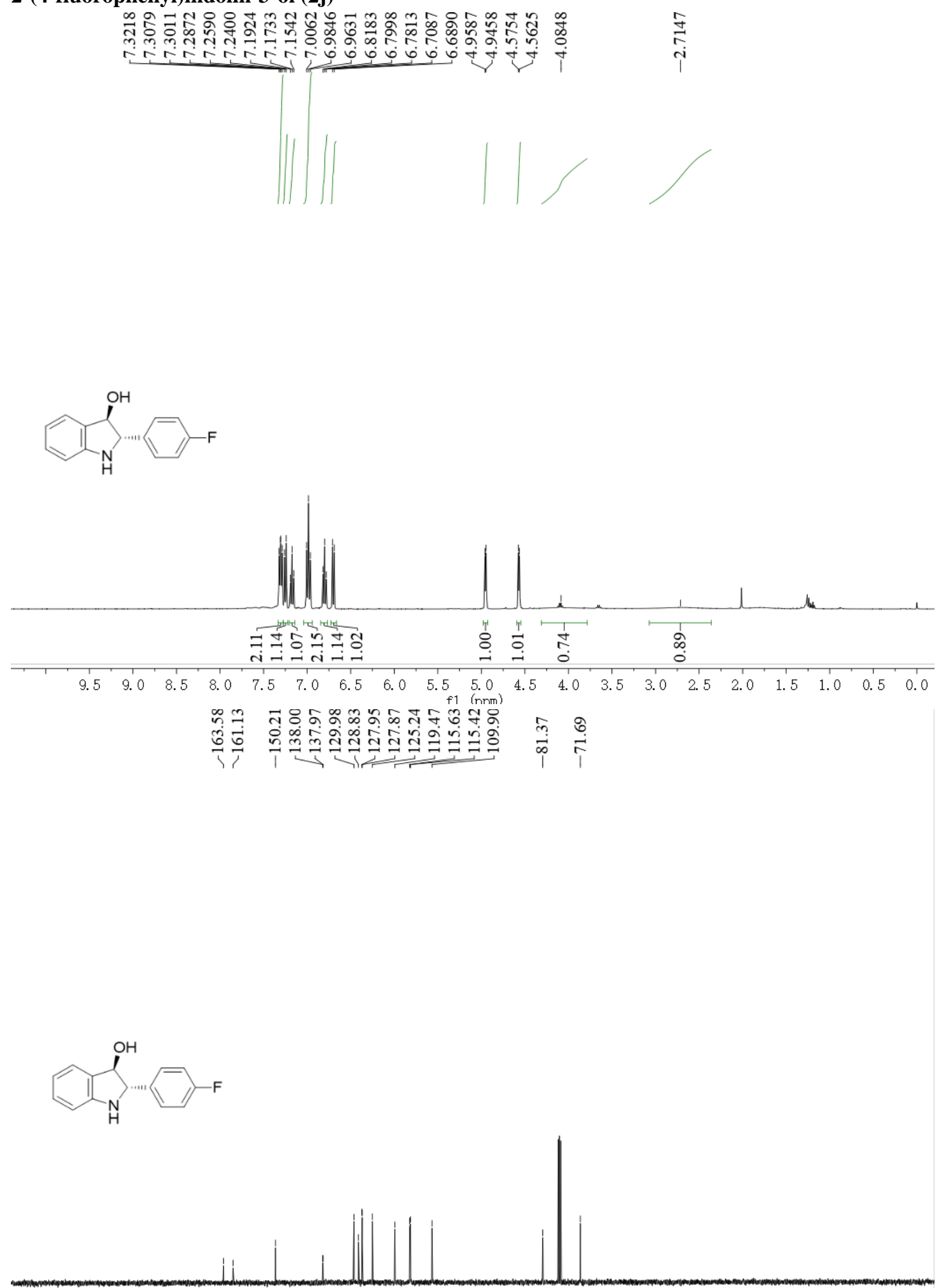

$\begin{array}{lllllllllllllllllllllll}210 & 200 & 190 & 180 & 170 & 160 & 150 & 140 & 130 & 120 & 110 & 100 & 90 & 80 & 70 & 60 & 50 & 40 & 30 & 20 & 10 & 0 & -10\end{array}$ 


\section{2-(4-chlorophenyl)indolin-3-ol (2k)}
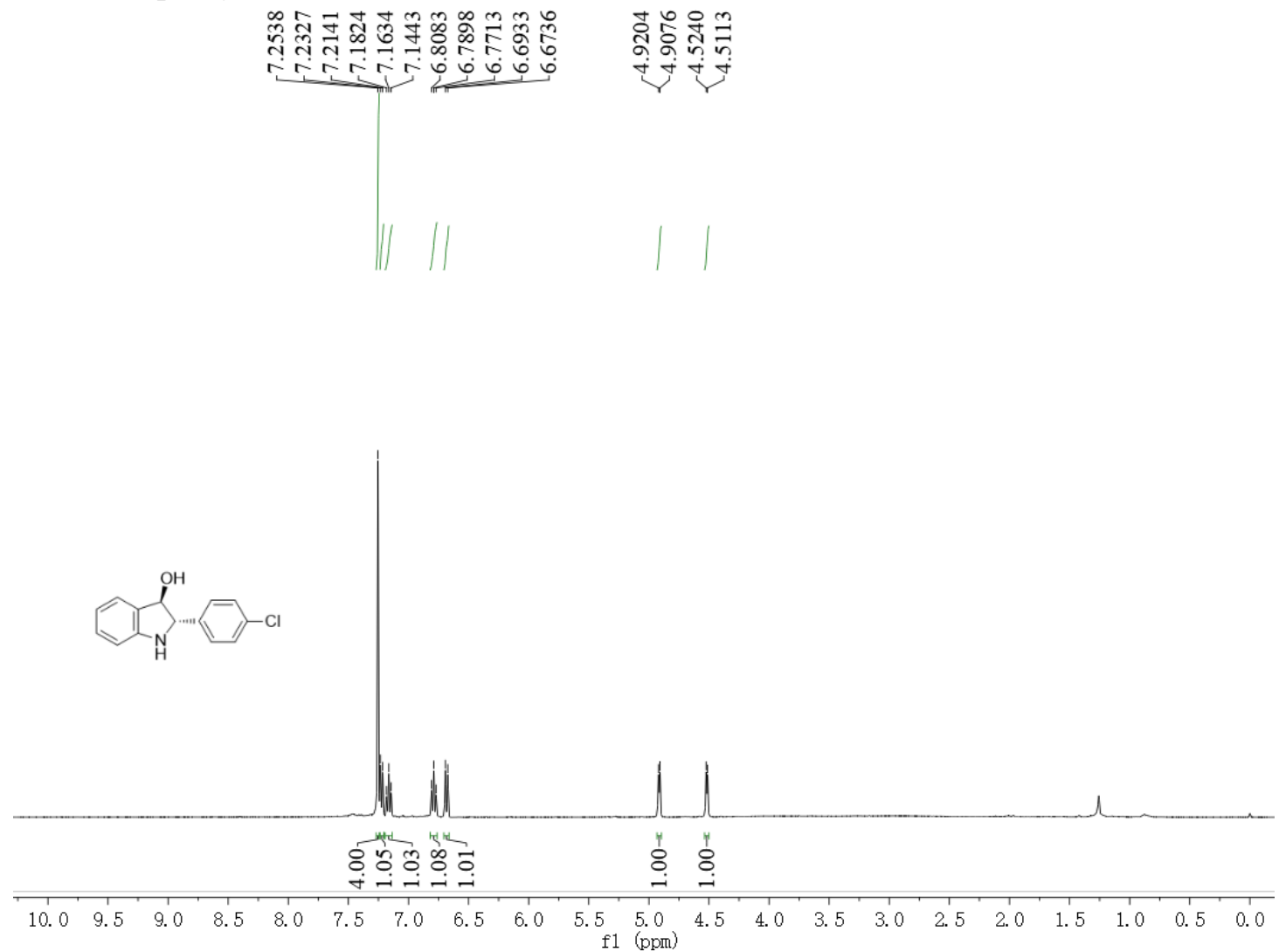

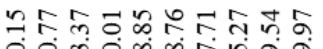

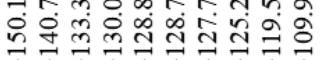

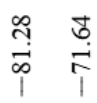
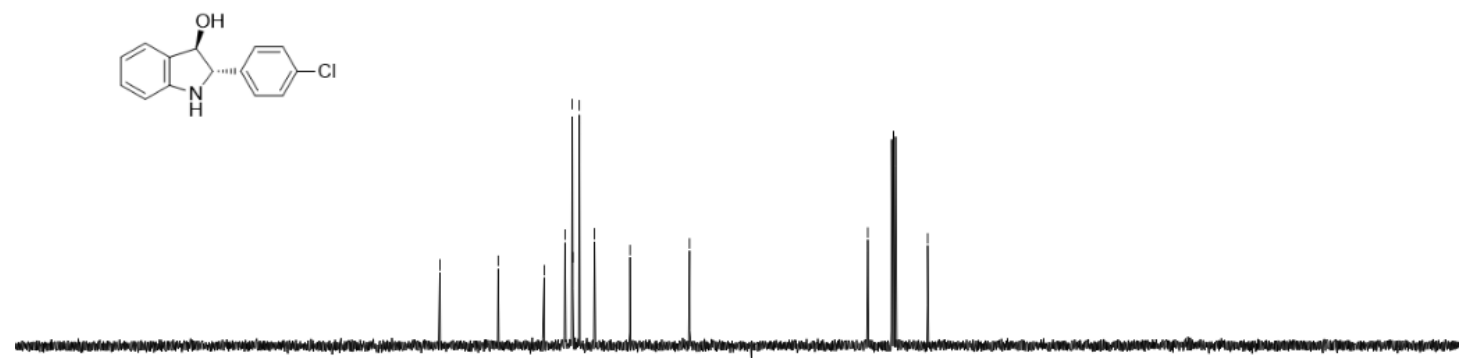

$\begin{array}{lllllllllllllllllllllllllllllllll}210 & 200 & 190 & 180 & 170 & 160 & 150 & 140 & 130 & 120 & 110 & 100 & 90 & 80 & 70 & 60 & 50 & 40 & 30 & 20 & 10 & 0 & -10\end{array}$ 
2-(4-(trifluoromethyl)phenyl)indolin-3-ol (2l)
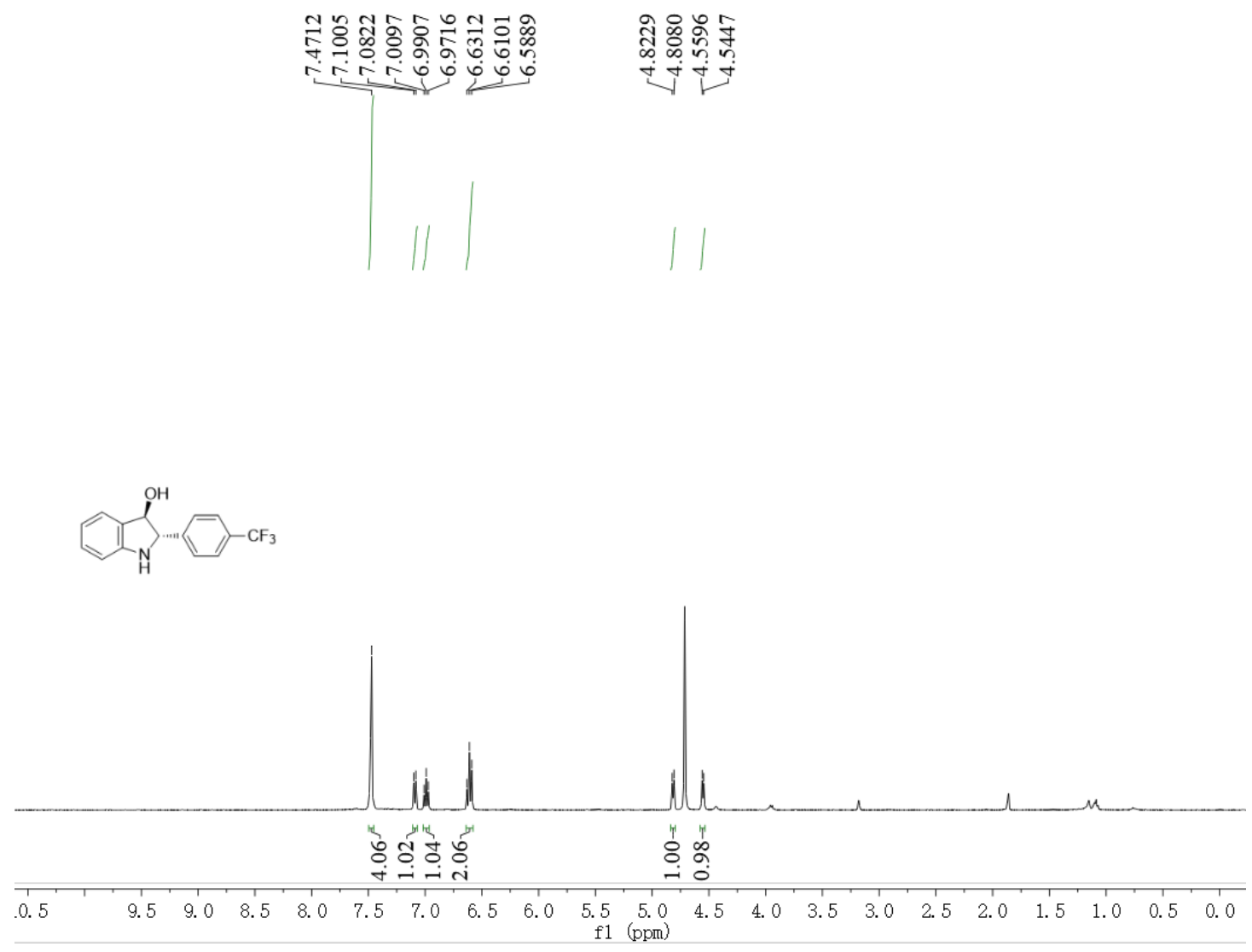

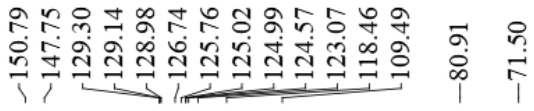

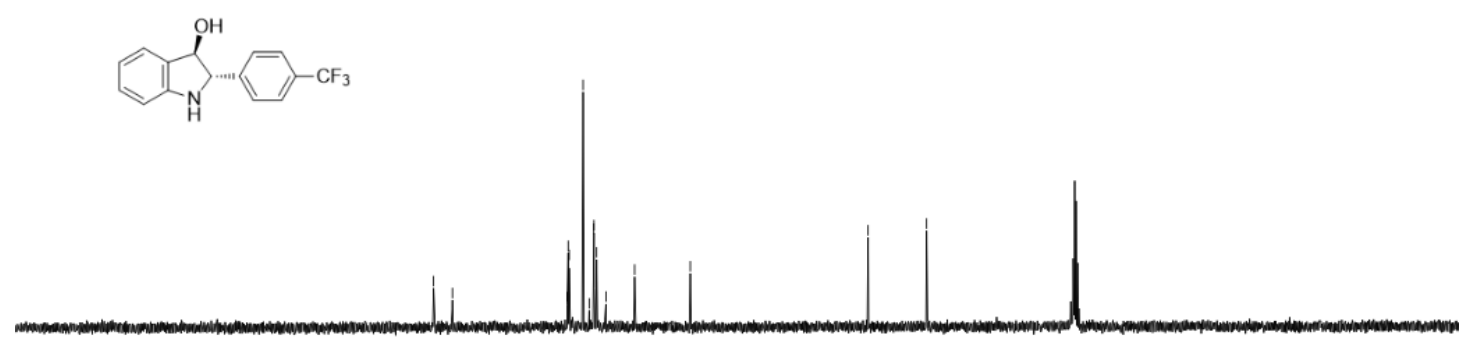

$\begin{array}{lllllllllllllllllllllllllllllll}210 & 200 & 190 & 180 & 170 & 160 & 150 & 140 & 130 & 120 & 110 & 100 & 90 & 80 & 70 & 60 & 50 & 40 & 30 & 20 & 10 & 0 & -10\end{array}$ 


\section{2-(naphthalen-1-yl)indolin-3-ol (2m)}
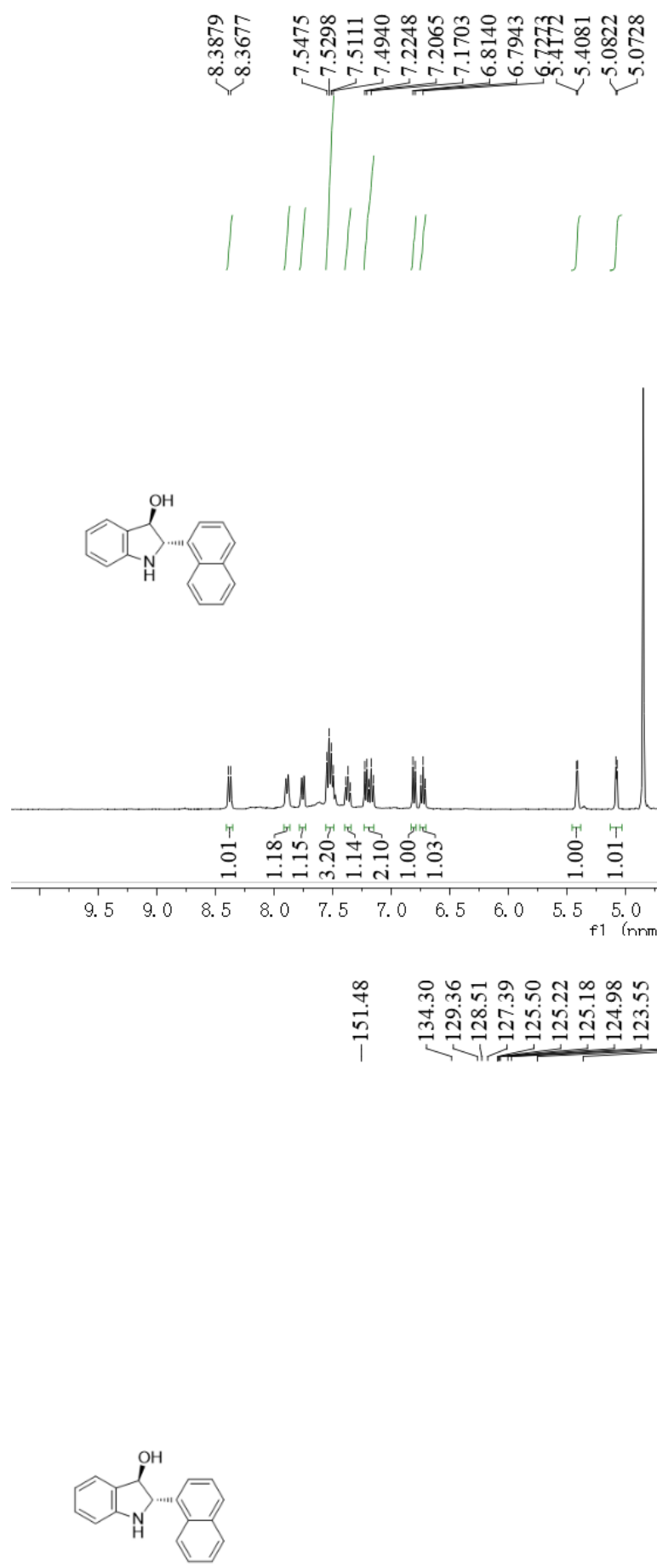

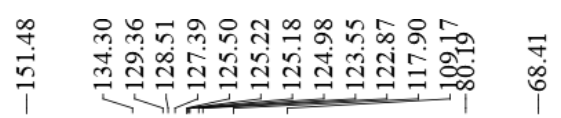
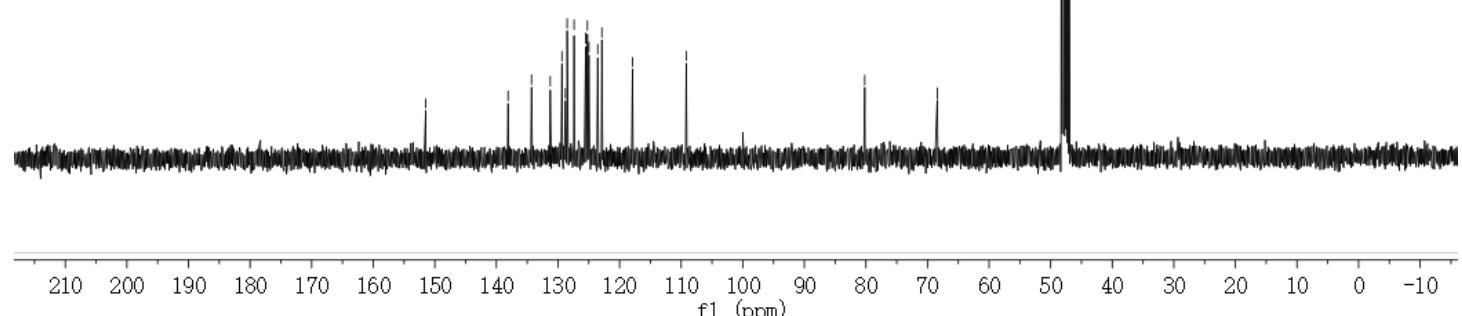


\section{2-(naphthalen-2-yl)indolin-3-ol (2n)}

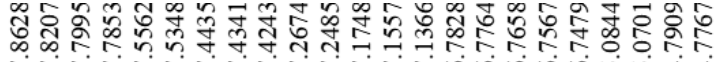

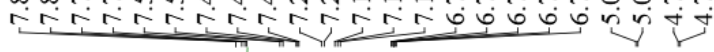
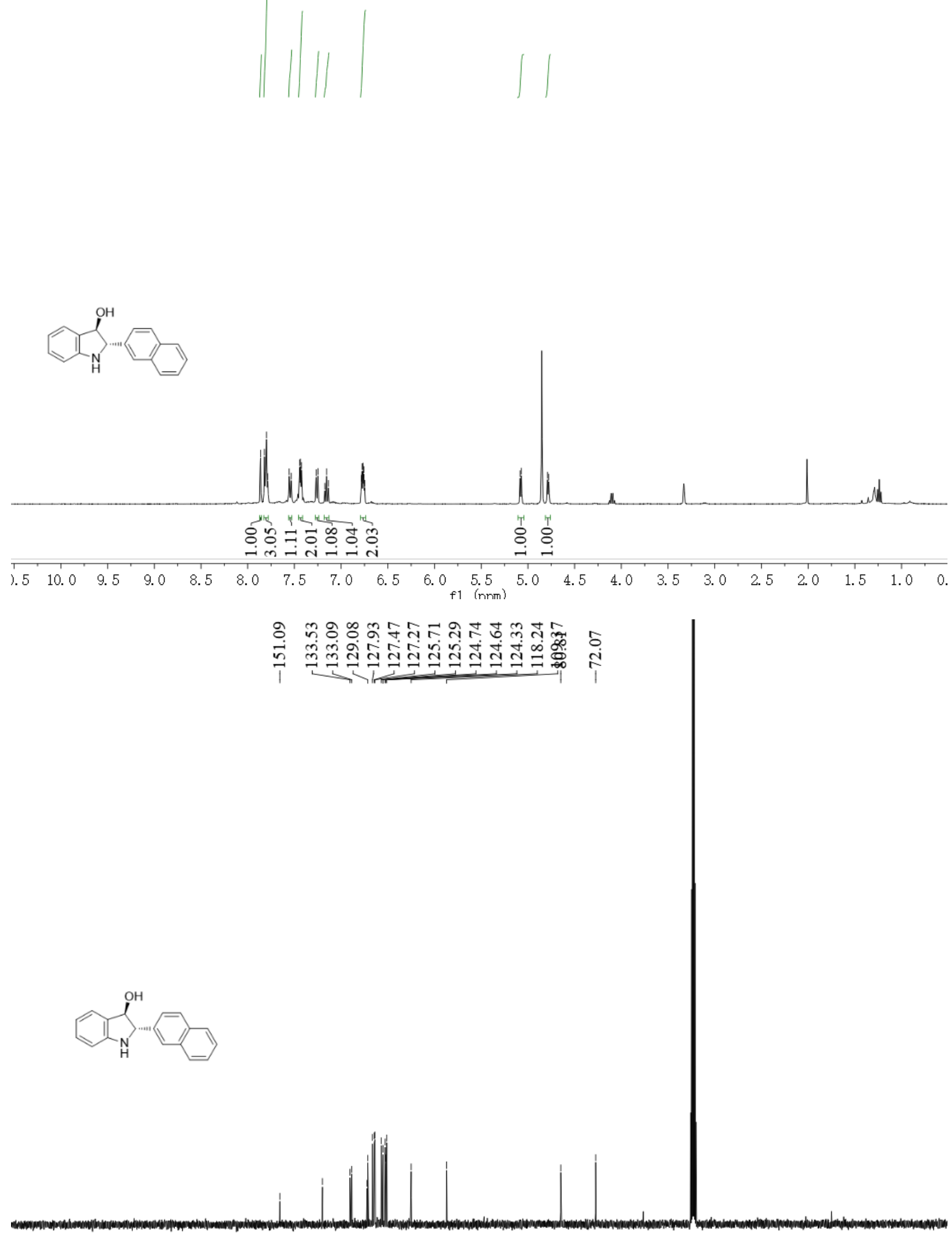

$\begin{array}{lllllllllllllllllllllll}210 & 200 & 190 & 180 & 170 & 160 & 150 & 140 & 130 & 120 & 110 & 100 & 90 & 80 & 70 & 60 & 50 & 40 & 30 & 20 & 10 & 0 & -10\end{array}$ 


\section{2-(thiophen-2-yl)indolin-3-ol (2o)}

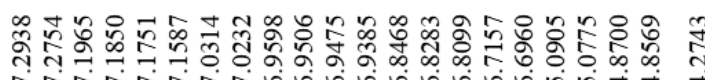

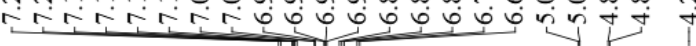

$\underset{\substack{\text { à } \\ \text { i }}}{ }$
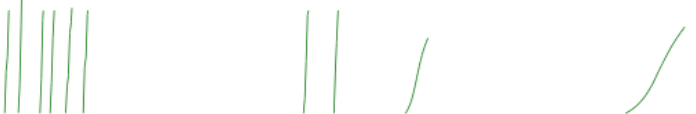

ST

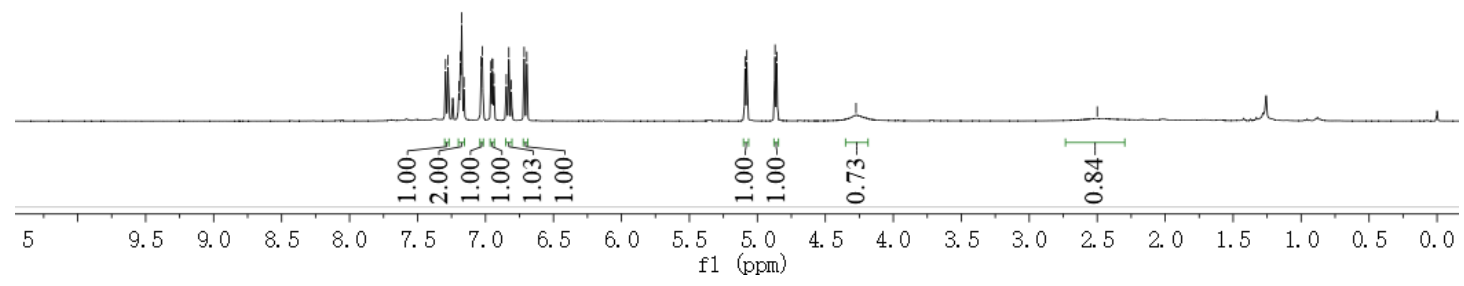

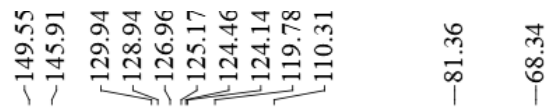
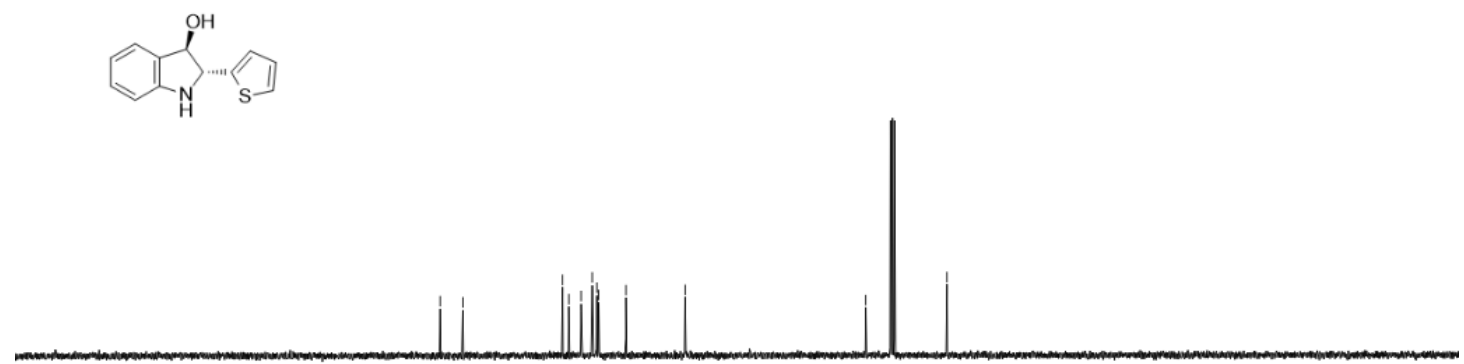

$\begin{array}{lllllllllllllllllllllllllllll}210 & 200 & 190 & 180 & 170 & 160 & 150 & 140 & 130 & 120 & 110 & 100 & 90 & 80 & 70 & 60 & 50 & 40 & 30 & 20 & 10 & 0 & -10\end{array}$ 
2-ethylindolin-3-ol (2p)

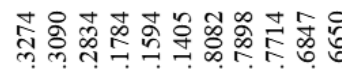

$\operatorname{Sin}_{H}^{O H}$

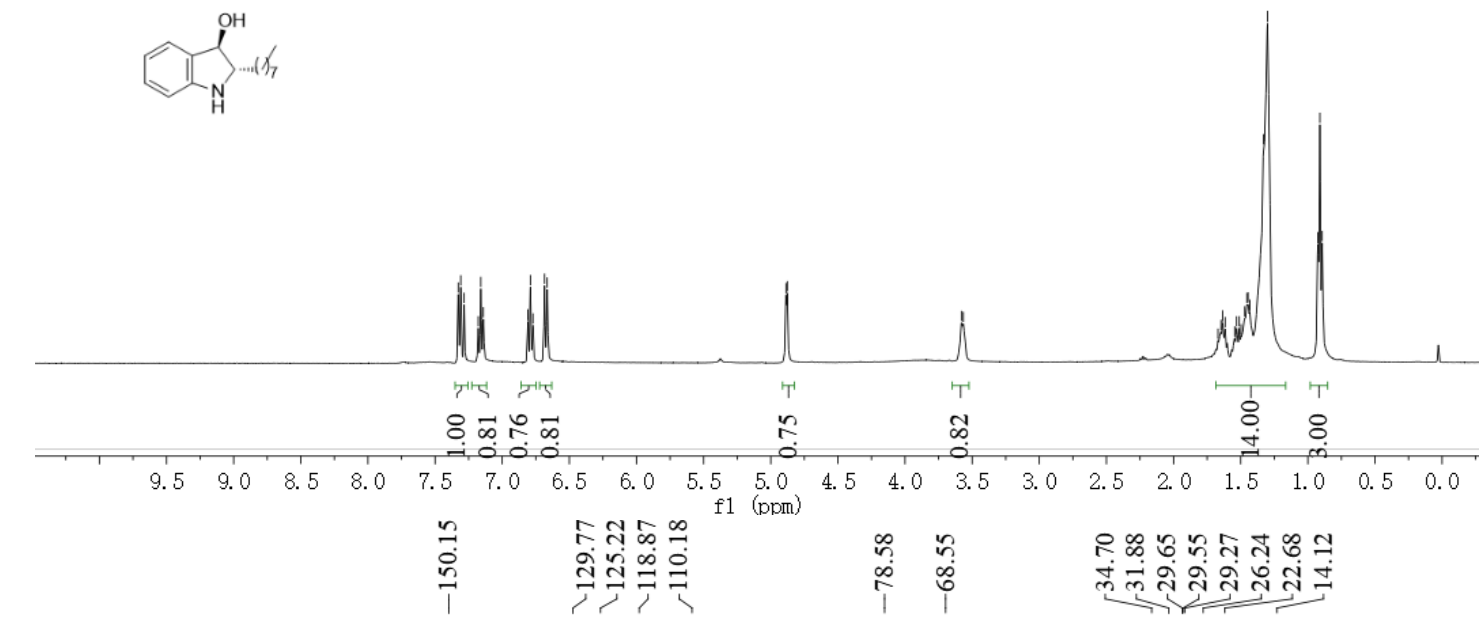

$\int_{H}^{O H}$

$\begin{array}{llllllllllllllllllllllll}210 & 200 & 190 & 180 & 170 & 160 & 150 & 140 & 130 & 120 & 110 & 100 & 90 & 80 & 70 & 60 & 50 & 40 & 30 & 20 & 10 & 0 & -10\end{array}$ f1 (ppm) 


\section{2-(tert-butyl)indolin-3-ol (2q)}

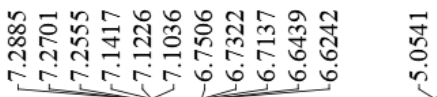

ले

भंग
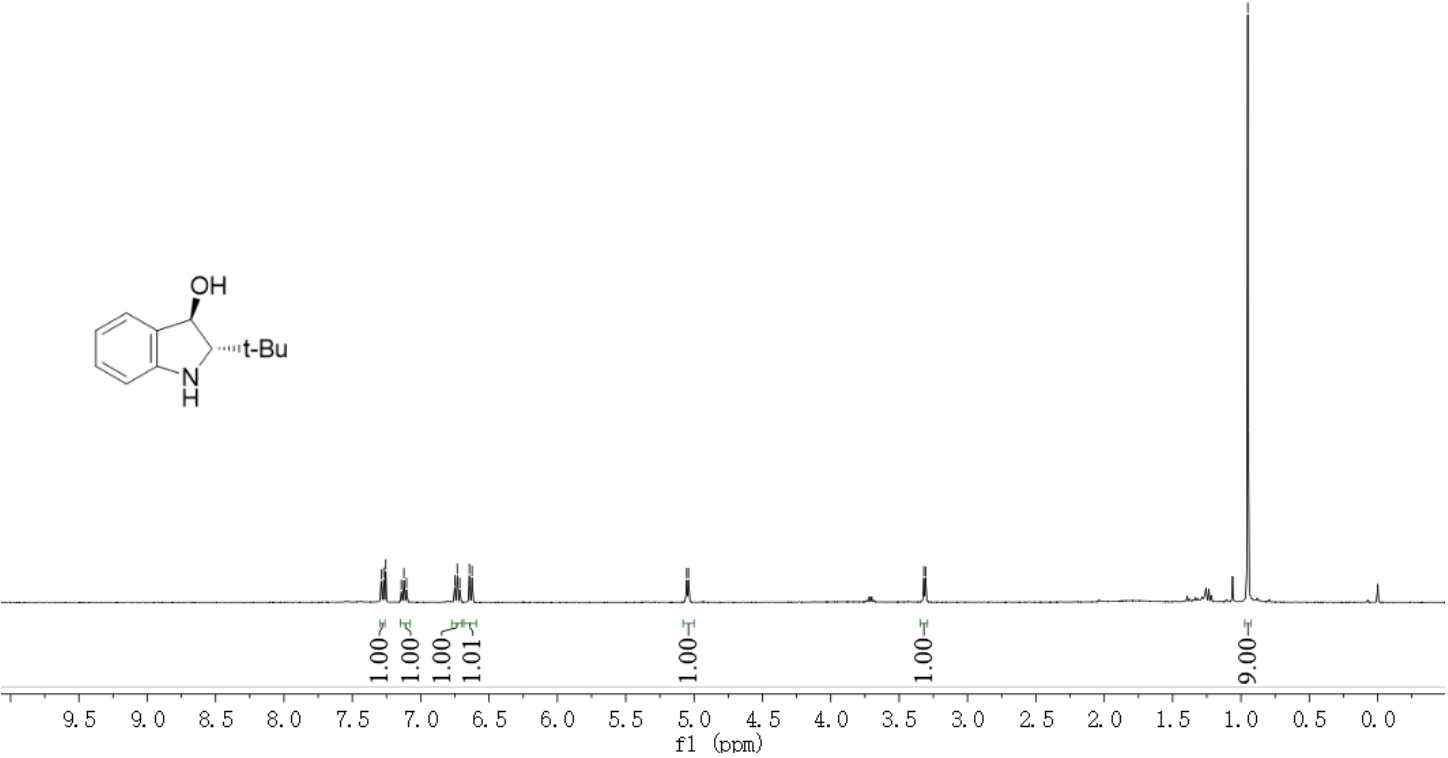

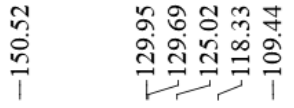
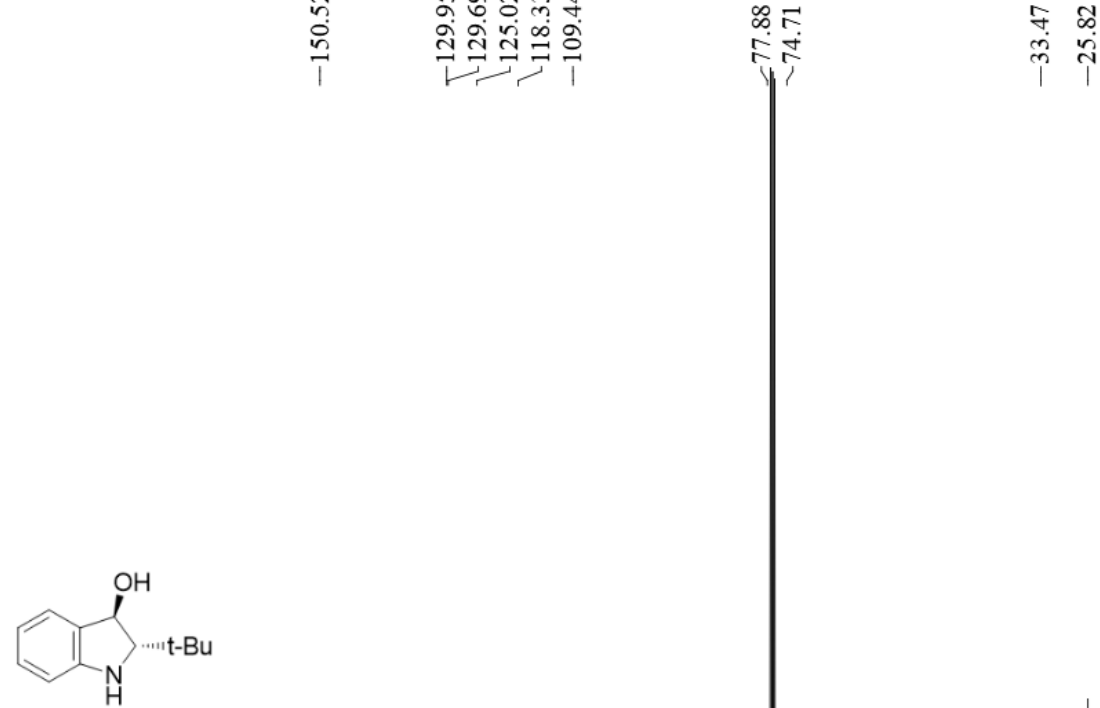

$\begin{array}{lllllllllllllllllllllll}210 & 200 & 190 & 180 & 170 & 160 & 150 & 140 & 130 & 120 & 110 & 100 & 90 & 80 & 70 & 60 & 50 & 40 & 30 & 20 & 10 & 0 & -10\end{array}$ 
6-chloro-2-(p-tolyl)indolin-3-ol (2r)

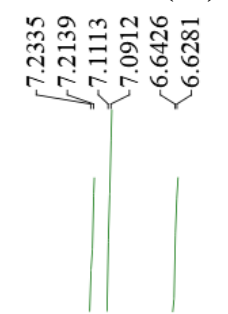

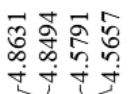

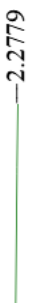

(N)

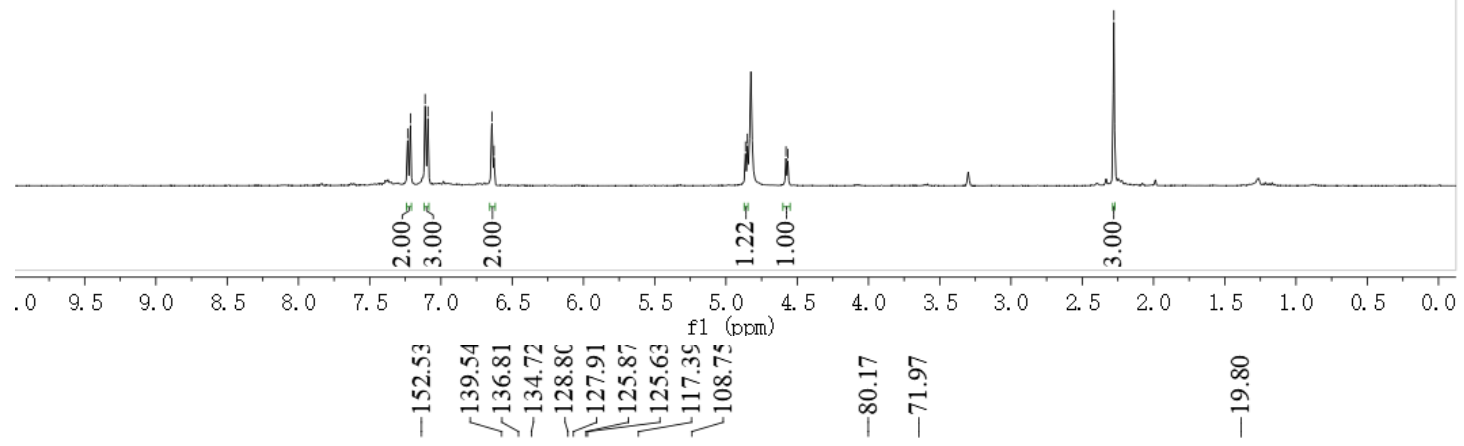

$\overbrace{\mathrm{H}}^{\mathrm{O}}$

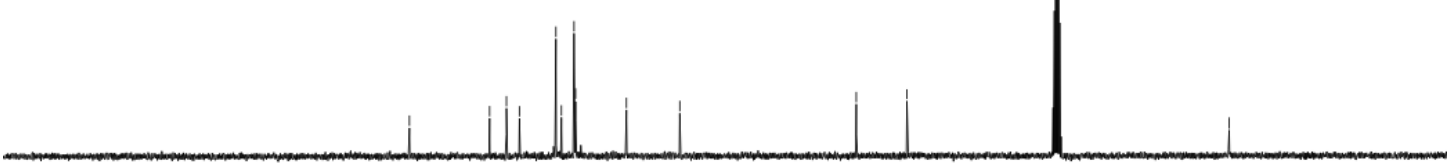

$\begin{array}{llllllllllllllllllllllllllll}210 & 200 & 190 & 180 & 170 & 160 & 150 & 140 & 130 & 120 & 110 & 100 & 90 & 80 & 70 & 60 & 50 & 40 & 30 & 20 & 10 & 0 & -10\end{array}$ 
6-methyl-2-(p-tolyl)indolin-3-ol (2s)
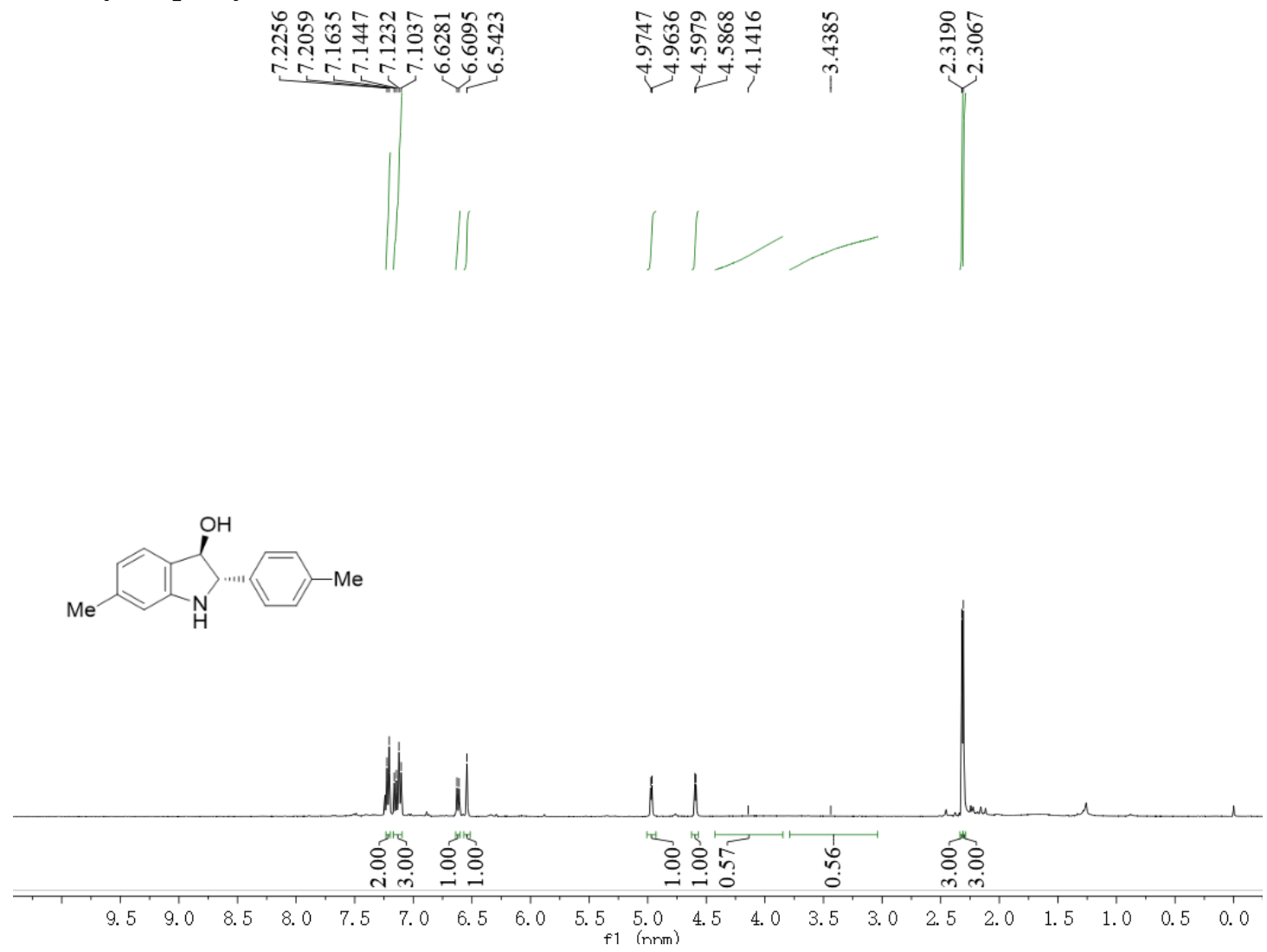

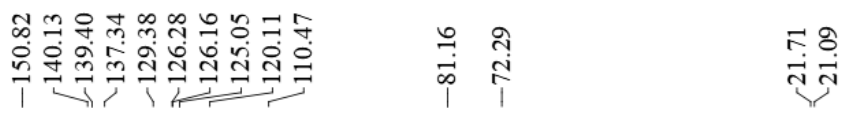

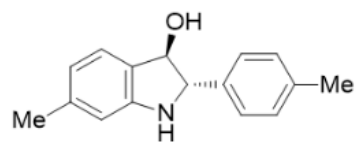

$\begin{array}{llllllllllllllllllllllll}1 & 200 & 190 & 180 & 170 & 160 & 150 & 140 & 130 & 120 & 110 & 100 & 90 & 80 & 70 & 60 & 50 & 40 & 30 & 20 & 10 & 0 & -10\end{array}$ 


\section{5-fluoro-2-(p-tolyl)indolin-3-ol (2t)}

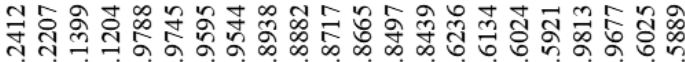

ririo
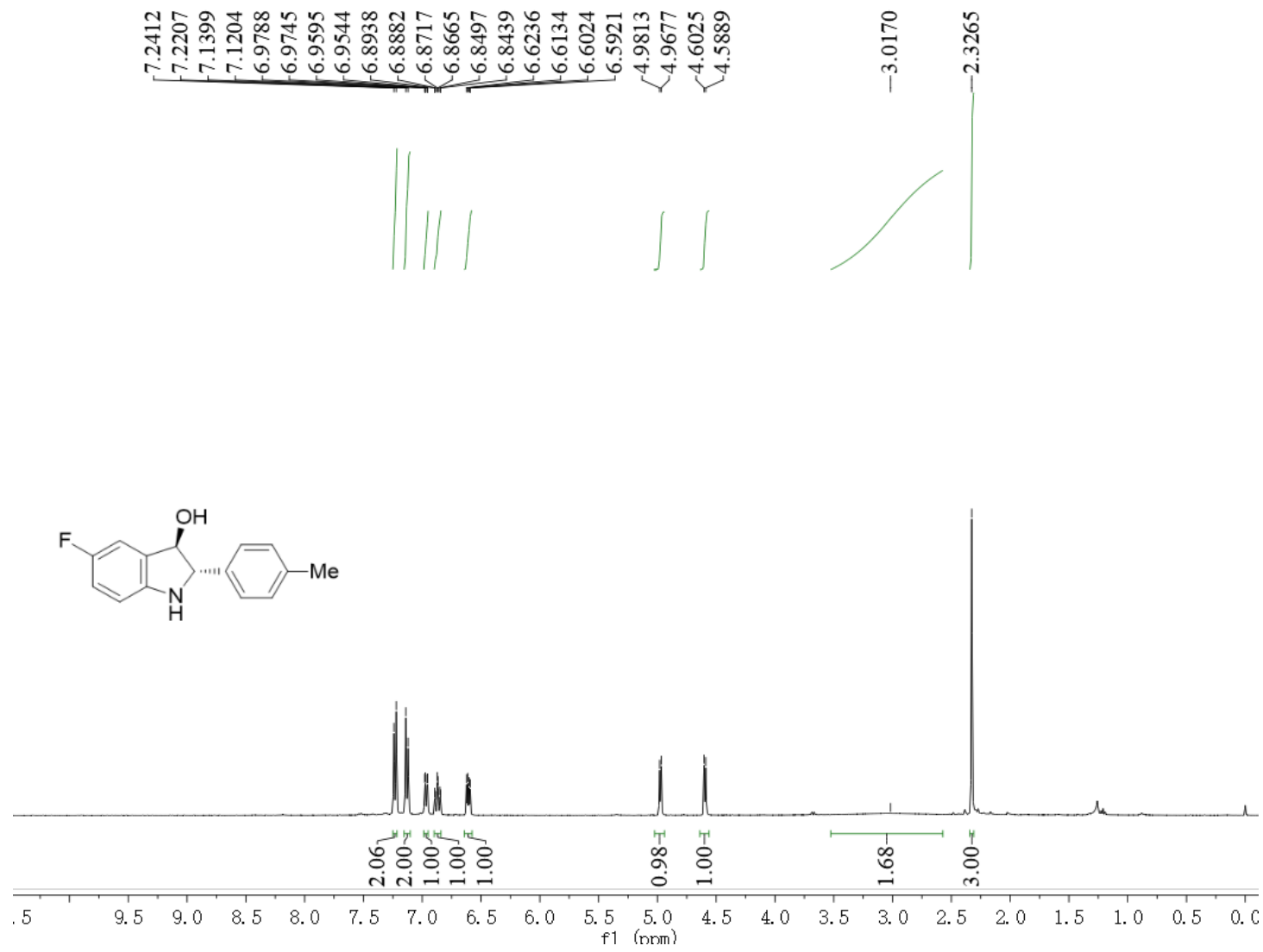

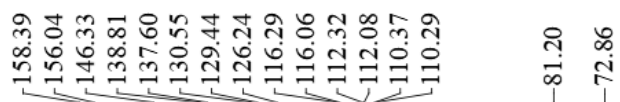

$\stackrel{0}{\stackrel{2}{1}}$

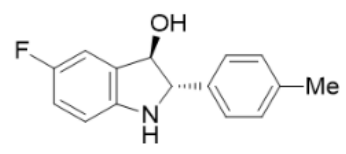

$\begin{array}{llllllllllllllllllllllll}210 & 200 & 190 & 180 & 170 & 160 & 150 & 140 & 130 & 120 & 110 & 100 & 90 & 80 & 70 & 60 & 50 & 40 & 30 & 20 & 10 & 0 & -10\end{array}$ 
2-(3-methoxyphenyl)-6-methylindolin-3-ol (2u)
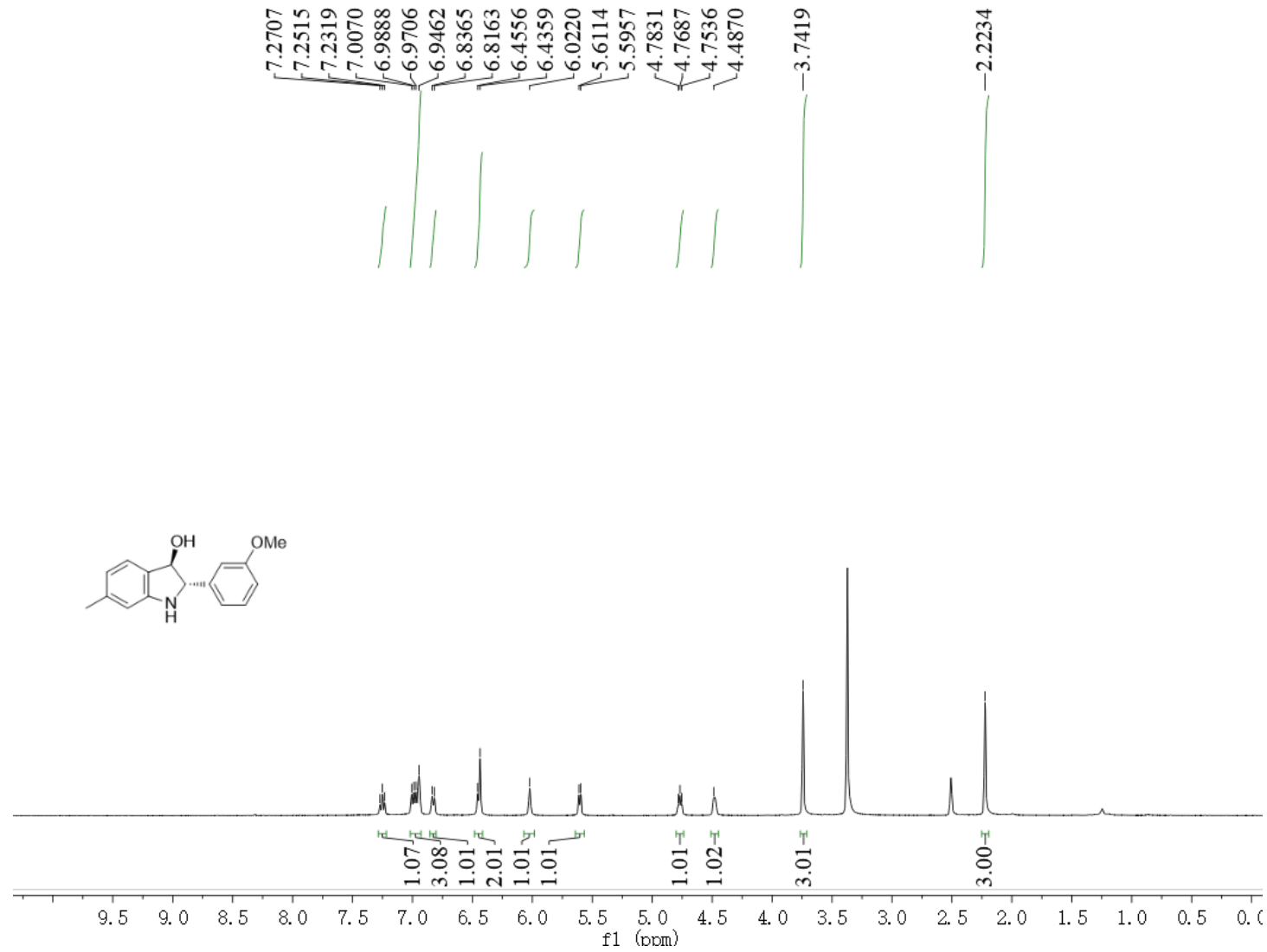

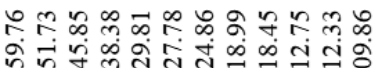

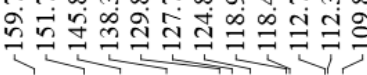

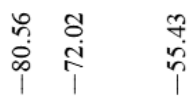

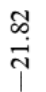

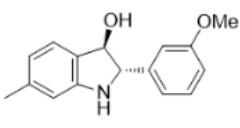

$\begin{array}{llllllllllllllllllllllllllll}210 & 200 & 190 & 180 & 170 & 160 & 150 & 140 & 130 & 120 & 110 & 100 & 90 & 80 & 70 & 60 & 50 & 40 & 30 & 20 & 10 & 0 & -10\end{array}$ 
6-chloro-2-(4-chlorophenyl)indolin-3-ol (2v)
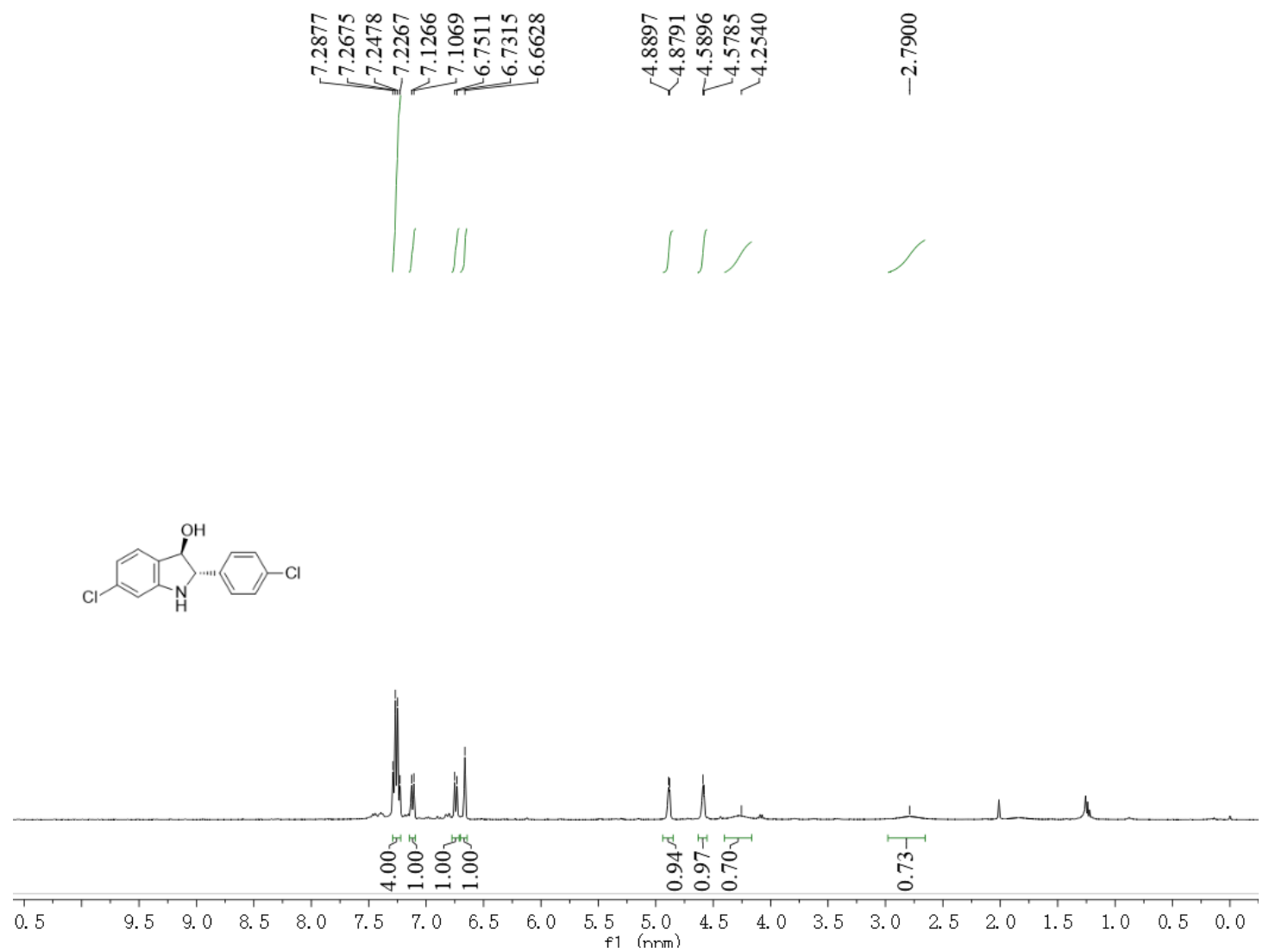

ஸุ

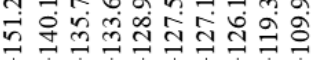
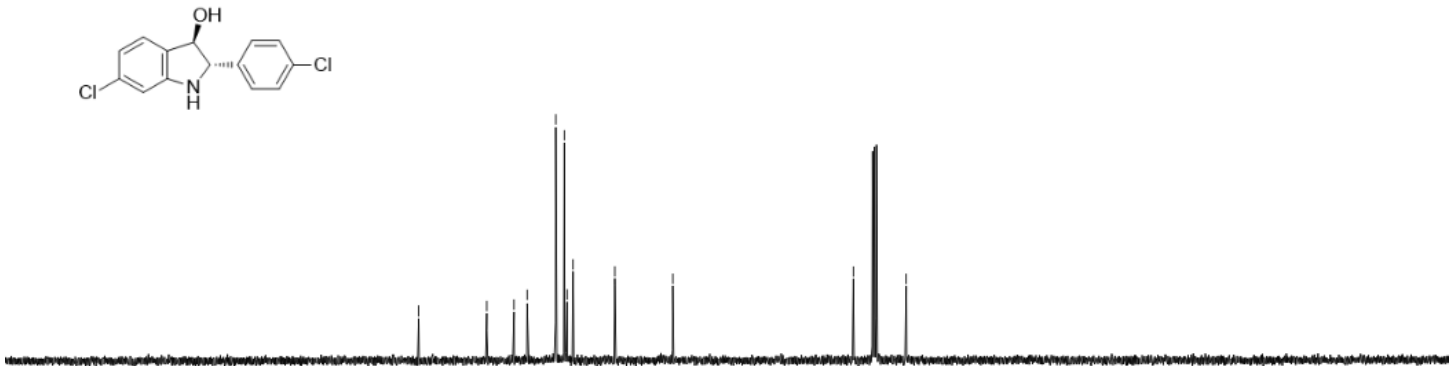

$\begin{array}{lllllllllllllllllllllll}210 & 200 & 190 & 180 & 170 & 160 & 150 & 140 & 130 & 120 & 110 & 100 & 90 & 80 & 70 & 60 & 50 & 40 & 30 & 20 & 10 & 0 & -10\end{array}$ $\mathrm{f} 1(\mathrm{nnm})$ 
2-(4-chlorophenyl)-6-methylindolin-3-ol (2w)

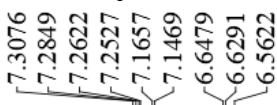

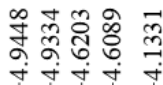

$\underset{\substack{n \\ \text { i }}}{i}$

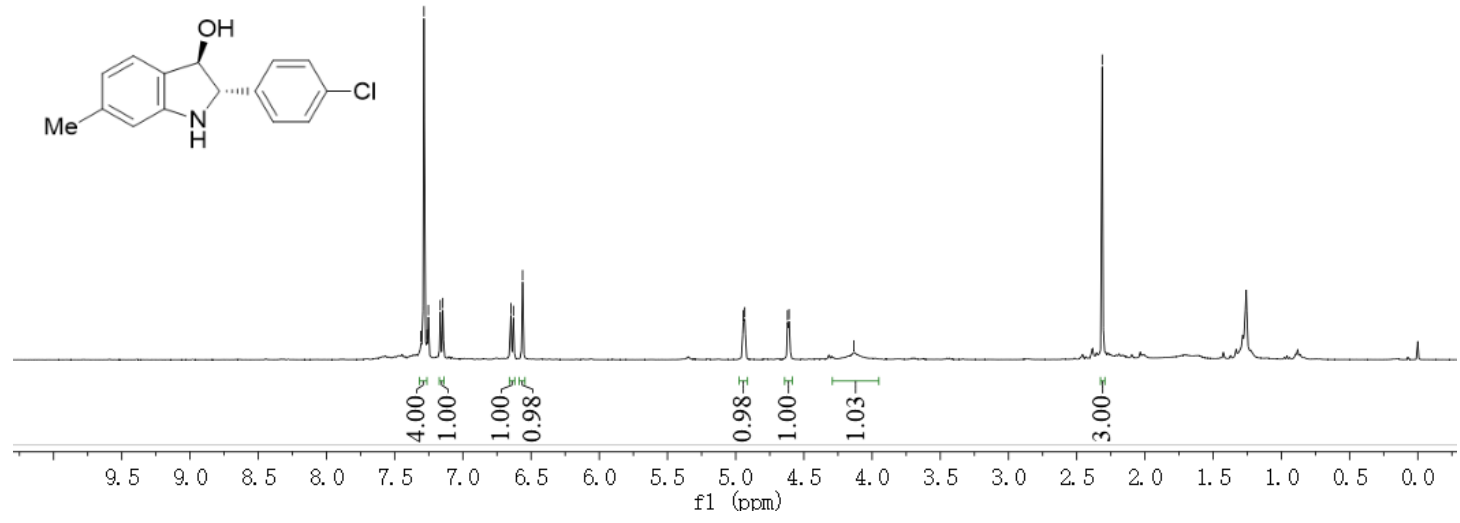

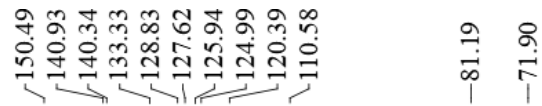

$\stackrel{i}{i}$<smiles>Cc1ccc2c(c1)N[C@H](c1ccc(Cl)cc1)C2O</smiles>

$\begin{array}{lllllllllllllllllllllll}210 & 200 & 190 & 180 & 170 & 160 & 150 & 140 & 130 & 120 & 110 & 100 & 90 & 80 & 70 & 60 & 50 & 40 & 30 & 20 & 10 & 0 & -10 \\ \mathrm{f} 1 & (\mathrm{nnm}) & & \end{array}$ 
2-(p-tolyl)-3H-indol-3-one (3a)

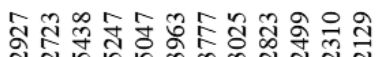

กิ

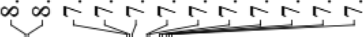
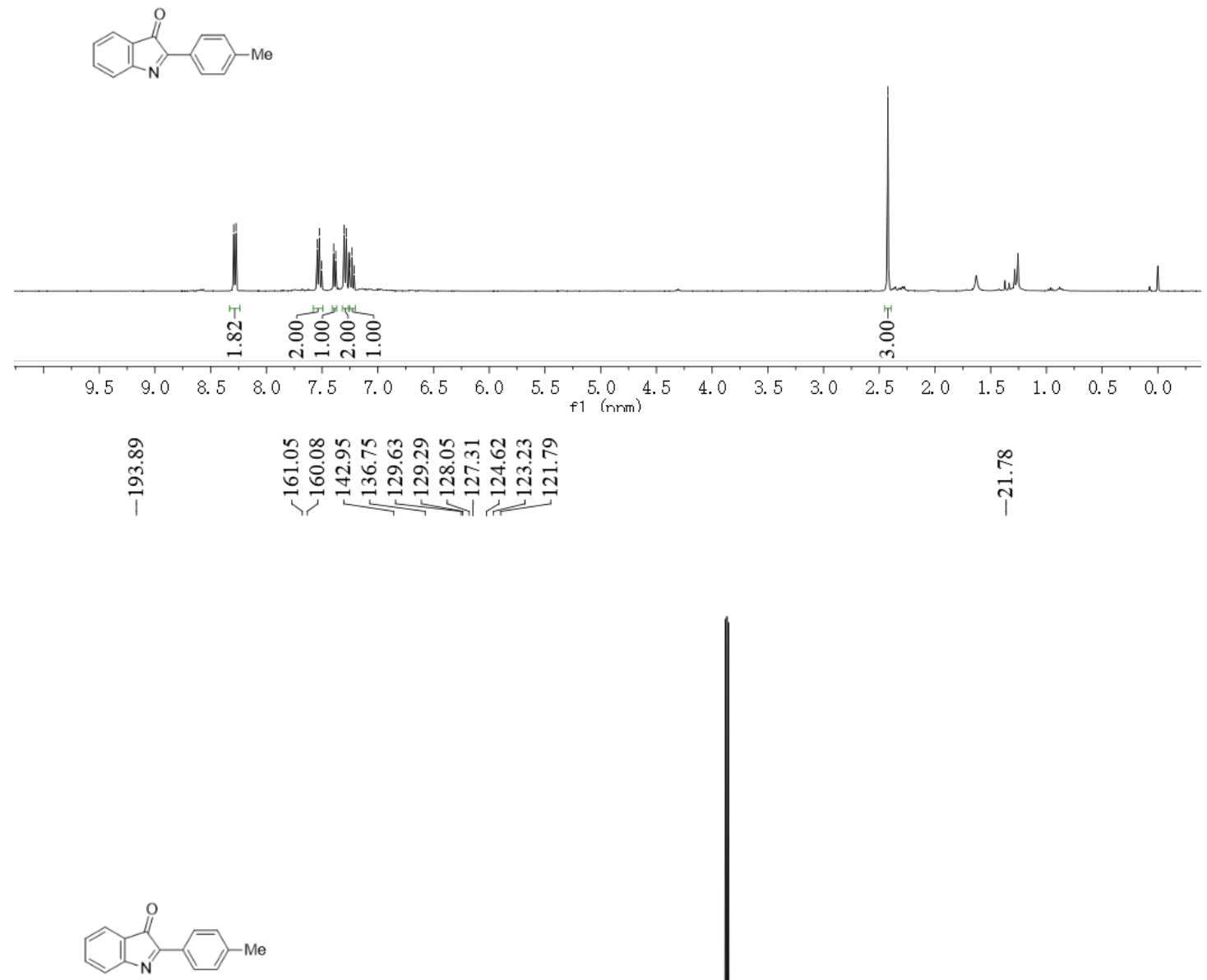

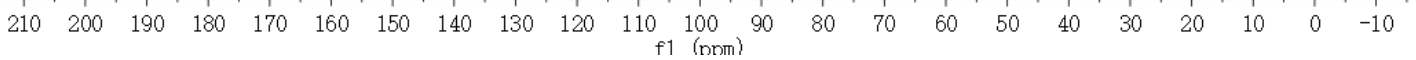


2-(4-methoxyphenyl)-3H-indol-3-one (3c)

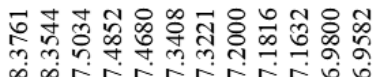

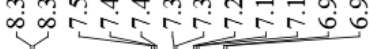
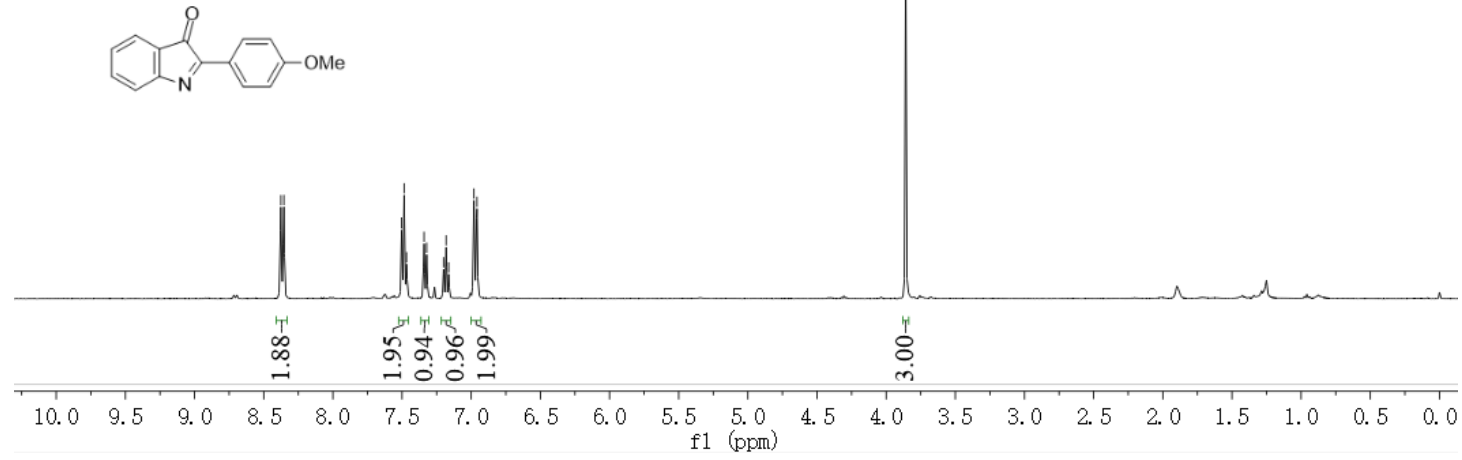

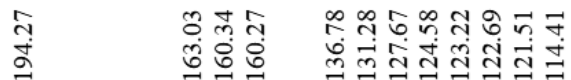

।
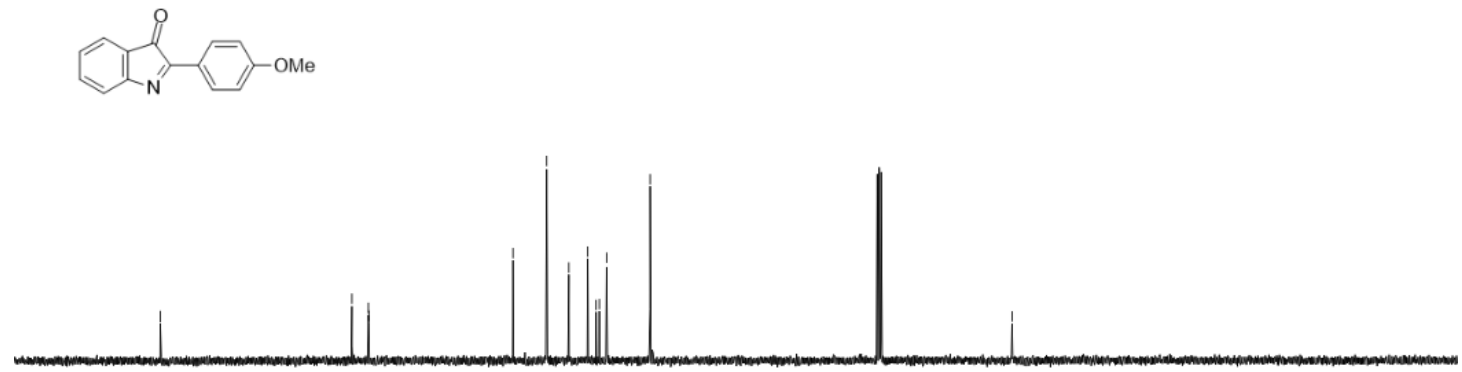

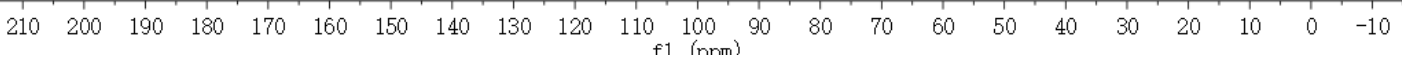


2-(4-ethylphenyl)-3H-indol-3-one(3e)

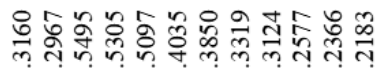
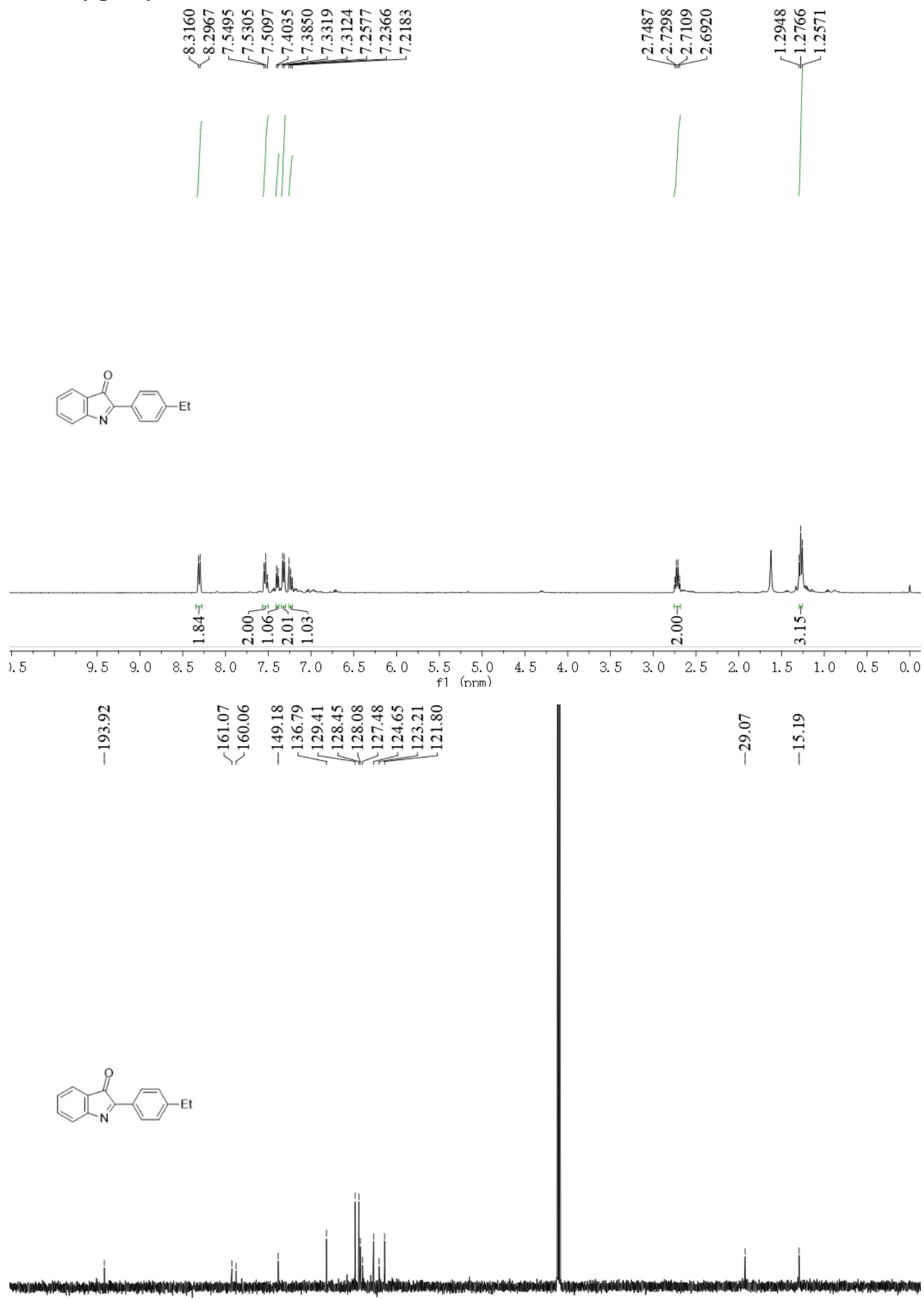

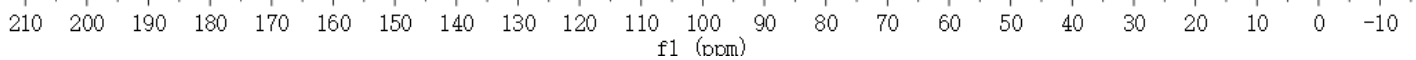


2-(3-methoxyphenyl)-3H-indol-3-one (3f)

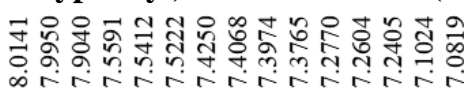
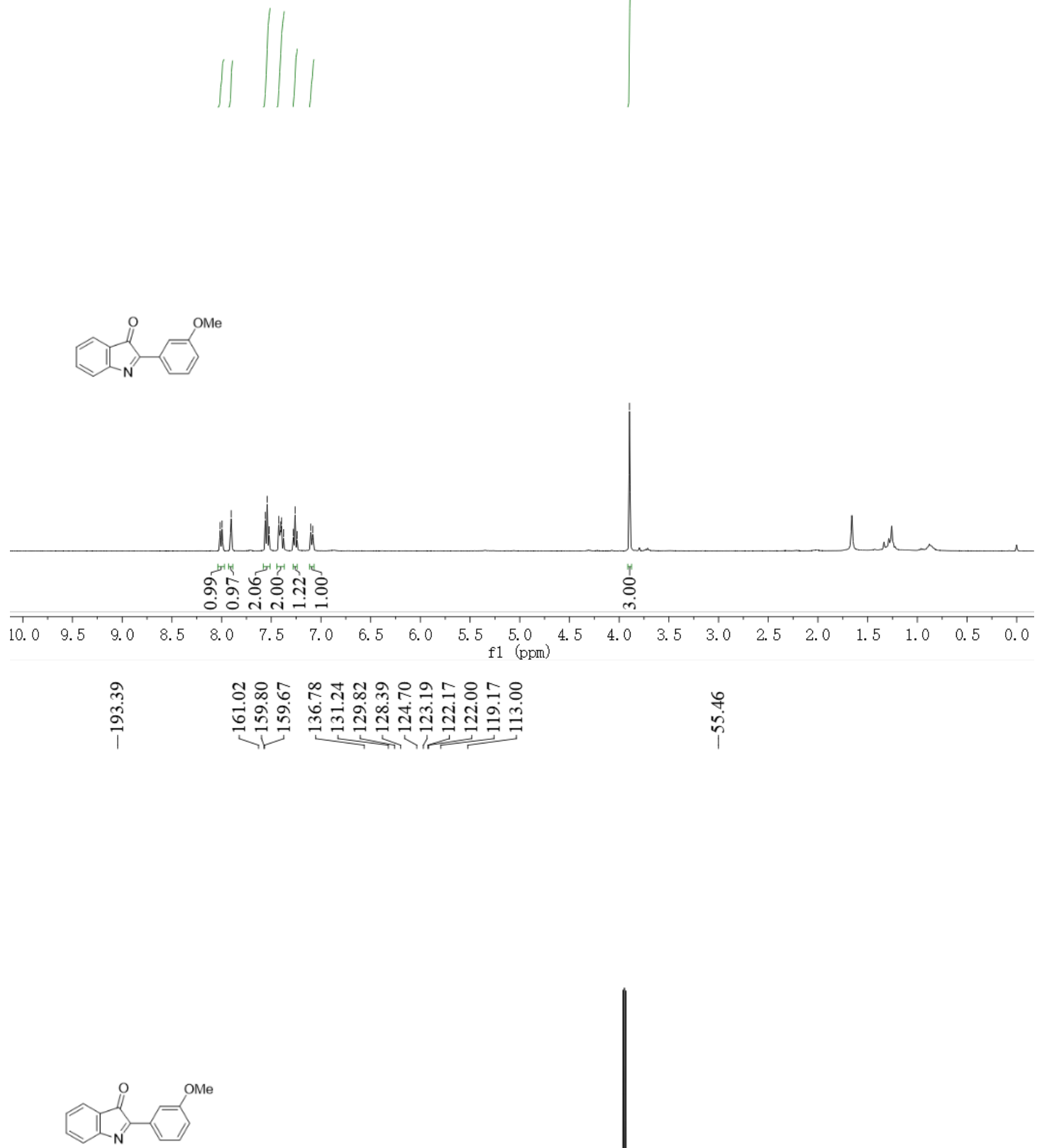

$\begin{array}{lllllllllllllllllllllllllllllll}210 & 200 & 190 & 180 & 170 & 160 & 150 & 140 & 130 & 120 & 110 & 100 & 90 & 80 & 70 & 60 & 50 & 40 & 30 & 20 & 10 & 0 & -10\end{array}$ 
2-(o-tolyl)-3H-indol-3-one (3h)
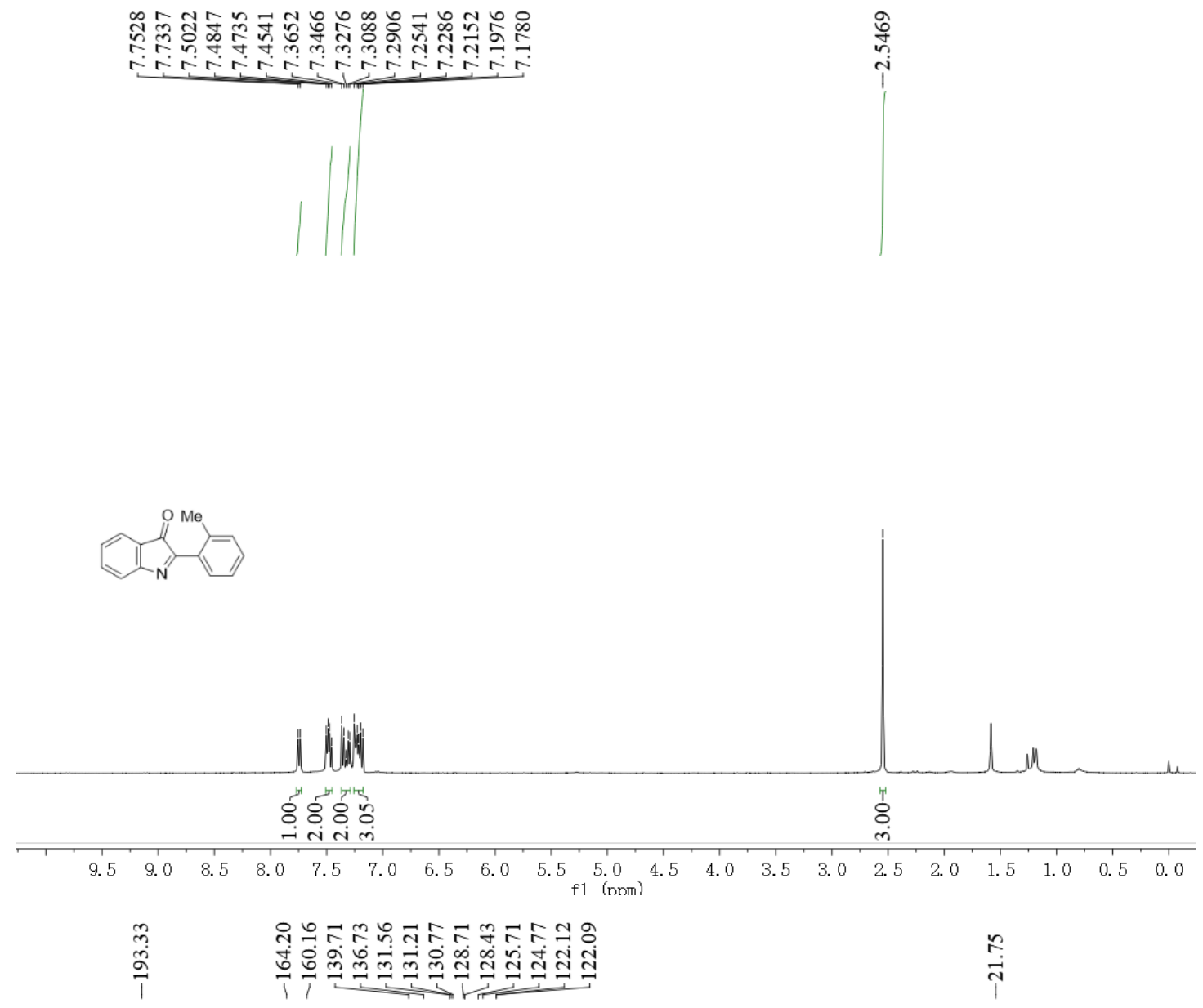

$\stackrel{n}{i}$

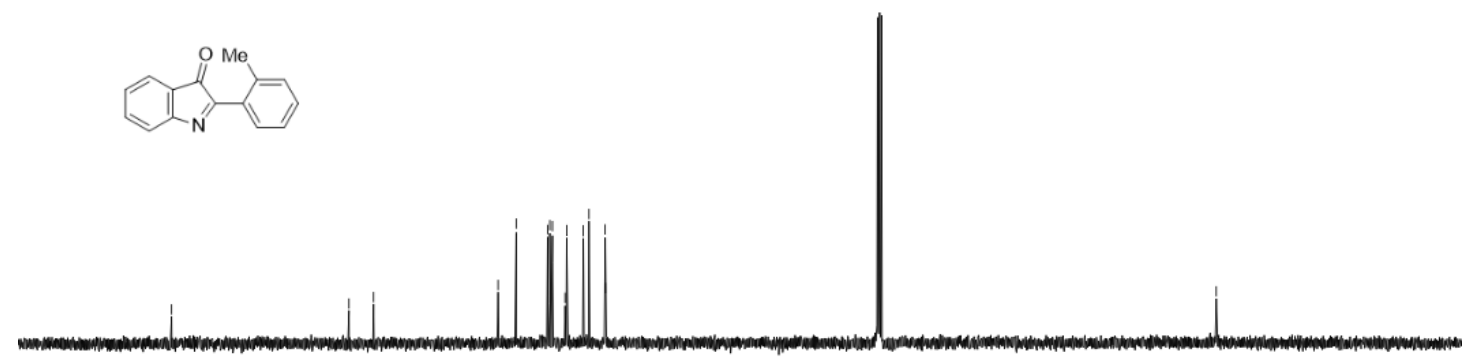

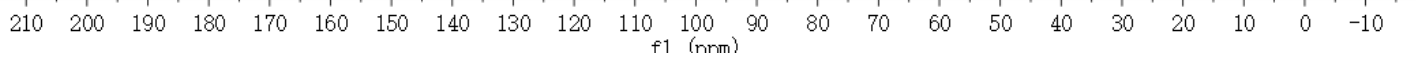


2-(naphthalen-1-yl)-3H-indol-3-one (3m)

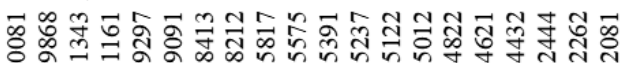

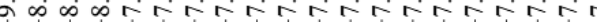
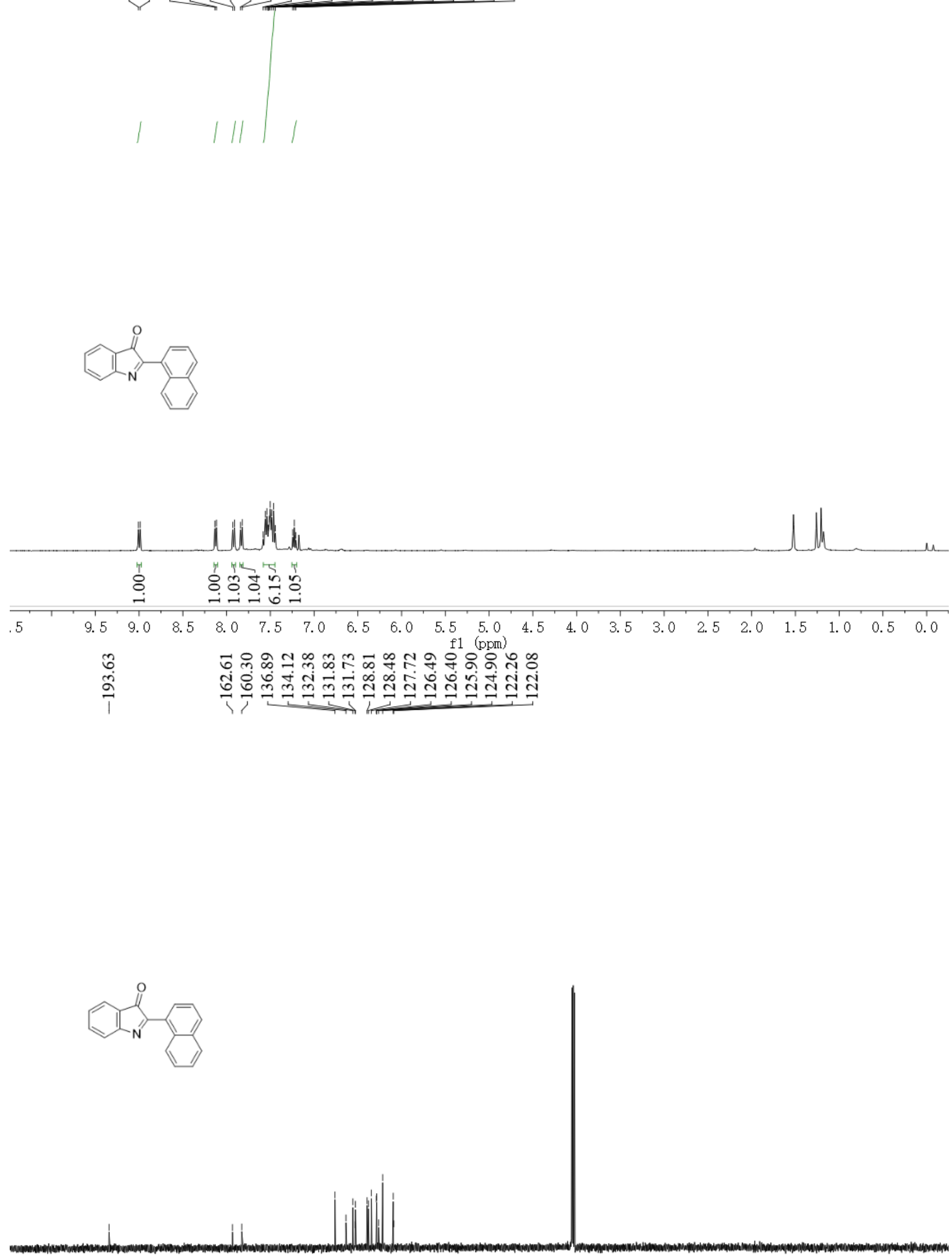

$\begin{array}{lllllllllllllllllllllll}210 & 200 & 190 & 180 & 170 & 160 & 150 & 140 & 130 & 120 & 110 & \underset{f 1}{100} \underset{(\mathrm{nnm})}{90} & 80 & 70 & 60 & 50 & 40 & 30 & 20 & 10 & 0 & -10\end{array}$ 
(E)-1-nitro-2-styrylbenzene (4)

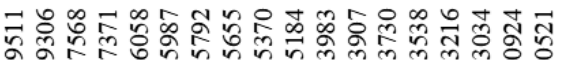

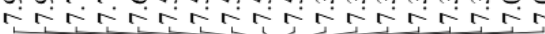
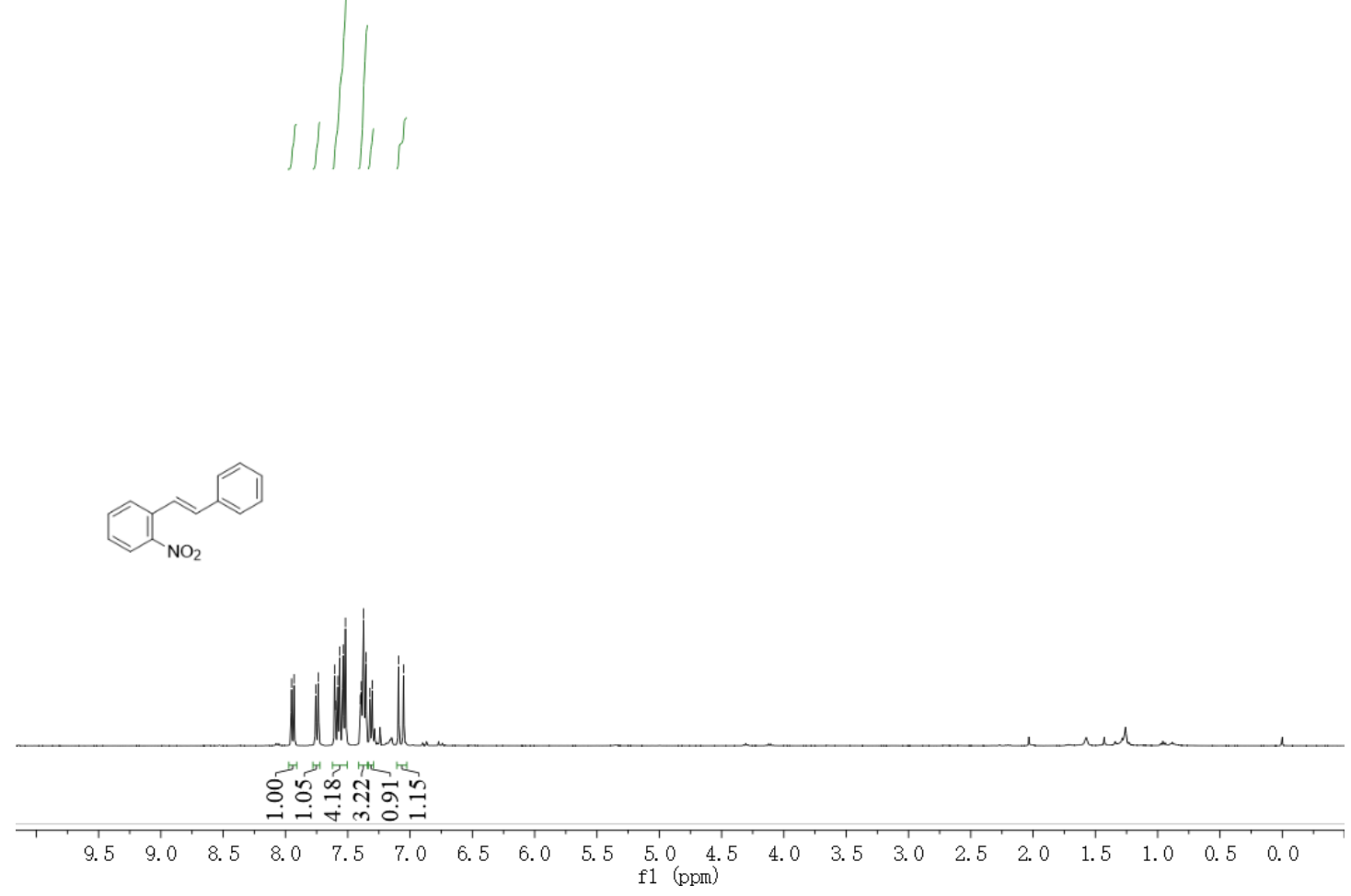

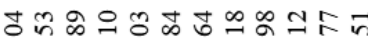

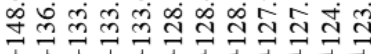

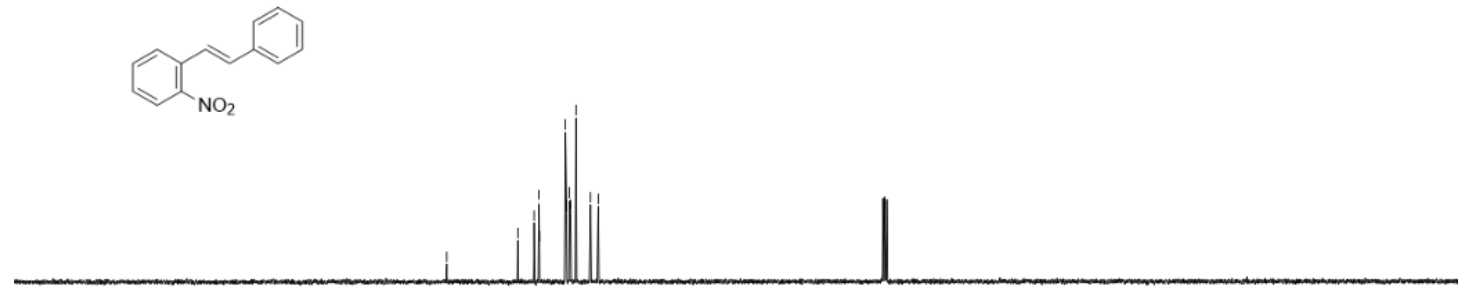

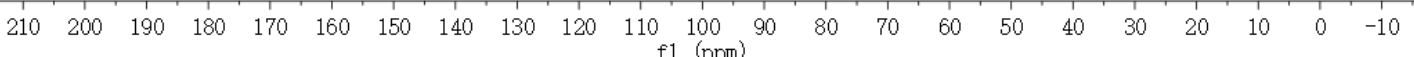


3-oxo-2-phenyl-3H-indole 1-oxide (5b)

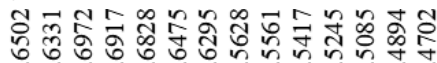

o on
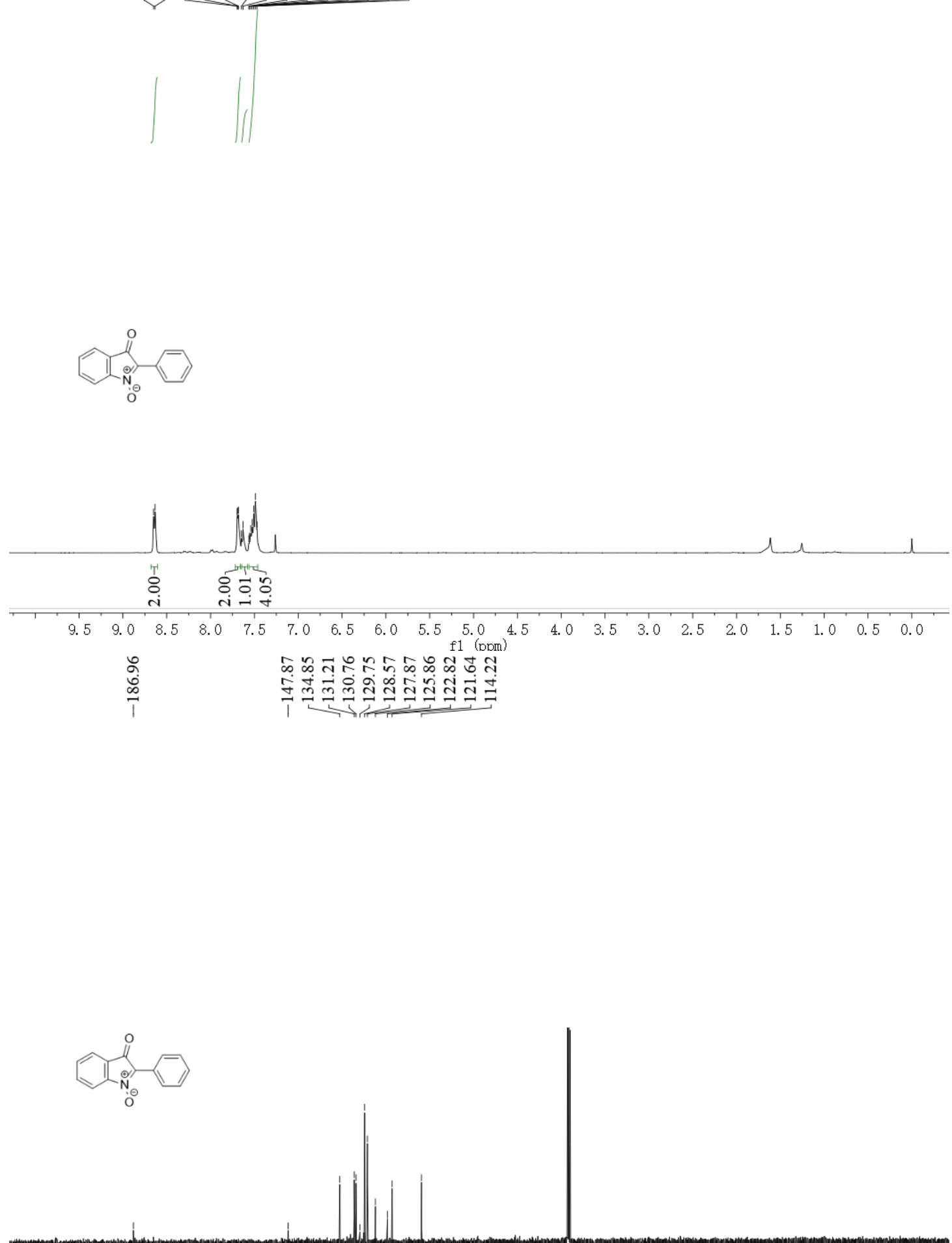

$\begin{array}{lllllllllllll}210 & 200 & 190 & 180 & 170 & 160 & 150 & 140 & 130 & 120 & 110 & 100 & 90\end{array}$ f1 (ppm) 